\title{
On conditional least squares estimation for affine diffusions based on continuous time observations
}

\author{
Beáta Bolyog*, Gyula PaP
}

Bolyai Institute, University of Szeged, Aradi vértanúk tere 1, H-6720 Szeged, Hungary

e-mails: bbeata@math.u-szeged.hu (B. Bolyog), papgy@math.u-szeged.hu (G. Pap).

* Corresponding author.

II Abstract

We study asymptotic properties of conditional least squares estimators for the drift parameters of two-factor affine diffusions based on continuous time observations. We distinguish three cases: subcritical, critical and supercritical. For all the drift parameters, in the subcritical and supercritical cases, asymptotic normality and asymptotic mixed normality is proved, while in the critical case, non-standard asymptotic behavior is described.

\section{Introduction}

Affine processes are applied in mathematical finance in several models including interest rate models (e.g. the Cox-Ingersoll-Ross, Vasiček or general affine term structure short rate models), option pricing (e.g. the Heston model) and credit risk models, see e.g. Duffie, Filipović and Schachermayer [16, Filipović [17], Baldeaux and Platen [2], and Alfonsi [1]. In this paper we consider two-factor affine processes, i.e. affine processes with state-space $[0, \infty) \times \mathbb{R}$. Dawson and Li [13] derived a jump-type stochastic differential equation (SDE) for such processes. Specializing this result to the diffusion case, i.e. two-factor affine processes without jumps, we obtain that for every $a \in[0, \infty), b, \alpha, \beta, \gamma \in \mathbb{R}$, $\sigma_{1}, \sigma_{2}, \sigma_{3} \in[0, \infty)$ and $\varrho \in[-1,1]$, the SDE

$$
\left\{\begin{array}{l}
\mathrm{d} Y_{t}=\left(a-b Y_{t}\right) \mathrm{d} t+\sigma_{1} \sqrt{Y_{t}} \mathrm{~d} W_{t}, \\
\mathrm{~d} X_{t}=\left(\alpha-\beta Y_{t}-\gamma X_{t}\right) \mathrm{d} t+\sigma_{2} \sqrt{Y_{t}}\left(\varrho \mathrm{d} W_{t}+\sqrt{1-\varrho^{2}} \mathrm{~d} B_{t}\right)+\sigma_{3} \mathrm{~d} L_{t},
\end{array} \quad t \in[0, \infty),\right.
$$

with an arbitrary initial value $\left(Y_{0}, X_{0}\right)$ with $\mathbb{P}\left(Y_{0} \in[0, \infty)\right)=1$ and independent of a 3 -dimensional standard Wiener process $\left(W_{t}, B_{t}, L_{t}\right)_{t \in[0, \infty)}$, has a pathwise unique strong solution being a twofactor affine diffusion process, and conversely, every two-factor affine diffusion process is a pathwise unique strong solution of a SDE (1.1) with appropriate parameters $a \in[0, \infty), \quad b, \alpha, \beta, \gamma \in \mathbb{R}$, $\sigma_{1}, \sigma_{2}, \sigma_{3} \in[0, \infty)$ and $\varrho \in[-1,1]$.

In this paper we study asymptotic properties of conditional least squares estimators (CLSE) $\left(\widehat{a}_{T}, \widehat{b}_{T}, \widehat{\alpha}_{T}, \widehat{\beta}_{T}, \widehat{\gamma}_{T}\right)$ of the drift parameters $(a, b, \alpha, \beta, \gamma)$ based on continuous time observations $\left(Y_{t}, X_{t}\right)_{t \in[0, T]}$ with $T>0$. This estimator is the high frequency limit in probability as $n \rightarrow \infty$ of the CLSE based on discrete time observations $\left(Y_{k / n}, X_{k / n}\right)_{k \in\{0, \ldots,\lfloor n T\rfloor\}}, n \in \mathbb{N}$. We do not estimate the parameters $\sigma_{1}, \sigma_{2}, \sigma_{3}$ and $\varrho$, since for all $T \in(0, \infty)$, they are measurable functions (i.e., statistics) of $\left(Y_{t}, X_{t}\right)_{t \in[0, T]}$, see Appendix C] It will turn out that for the calculation of $\left(\widehat{a}_{T}, \widehat{b}_{T}, \widehat{\alpha}_{T}, \widehat{\beta}_{T}, \widehat{\gamma}_{T}\right)$ one does not need to know the values of the diffusion coefficients $\sigma_{1}, \sigma_{2}, \sigma_{3}$ and $\varrho$, see (3.6).

2010 Mathematics Subject Classifications: 60J60, 62F12.

Key words and phrases: affine processes, conditional least squares estimators. 
The first coordinate process $Y$ in (1.1) is called a Cox-Ingersoll-Ross (CIR) process (see Cox, Ingersoll and Ross [12]). In the submodel consisting only of the process $Y$, Overbeck and Rydén [27. Theorems 3.4, 3.5 and 3.6] derived the CLSE of $(a, b)$ based on continuous time observations $\left(Y_{t}\right)_{t \in[0, T]}$ with $T>0$, i.e., the limit in probability as $n \rightarrow \infty$ of the CLSE based on discrete

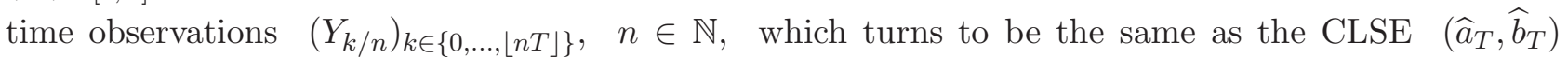
of $(a, b)$ based on continuous time observations $\left(Y_{t}, X_{t}\right)_{t \in[0, T]}$, and they proved strong consistency and asymptotic normality in case of a subcritical CIR process $Y$, i.e., when $b>0$ and the initial distribution is the unique stationary distribution of the model.

Barczy at al. [6] considered a submodel of (1.1) with $a \in(0, \infty), \quad \beta=0, \sigma_{1}=1, \quad \sigma_{2}=1$, $\varrho=0$ and $\sigma_{3}=0$. The estimator of the parameters $(\alpha, \gamma)$ based on continuous time observations $\left(X_{t}\right)_{t \in[0, T]}$ with $T>0$ (which they call a least square estimator) is in fact the CLSE, i.e., the limit in

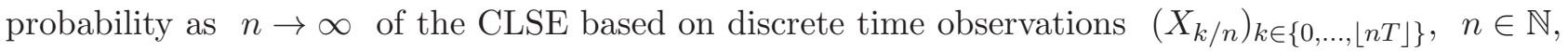
which can be shown by the method of the proof of Lemma 3.3. They proved strong consistency and asymptotic normality in case of a subcritical process $(Y, X)$, i.e., when $b>0$ and $\gamma>0$.

Barczy at al. [7] considered the so-called Heston model, which is a submodel of (1.1) with $a, \sigma_{1}, \sigma_{2} \in$ $(0, \infty), \quad \gamma=0, \varrho \in(-1,1)$ and $\sigma_{3}=0$. The estimator of the parameters $(a, b, \alpha, \beta)$ based on continuous time observations $\left(Y_{t}, X_{t}\right)_{t \in[0, T]}$ with $T>0$ (which they call least square estimator) is in fact the CLSE, i.e., the limit in probability as $n \rightarrow \infty$ of the CLSE based on discrete time

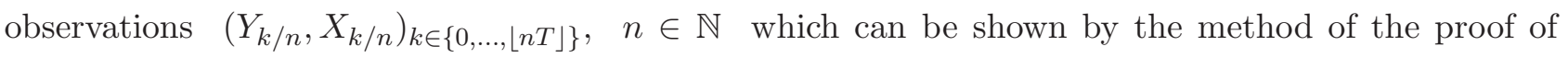
Lemma 3.3. They proved strong consistency and asymptotic normality in case of a subcritical process $(Y, X)$, i.e., when $b>0$. Note that Barczy and Pap [8] studied the maximum likelihood estimator (MLE) of the parameters $(a, b, \alpha, \beta)$ in this Heston model under the additional assumption $a \geqslant \frac{\sigma_{1}^{2}}{2}$. In the subcritical case, i.e., when $b>0$, they proved strong consistency and asymptotic normality of the MLE of $(a, b, \alpha, \beta)$ under the additional assumption $a>\frac{\sigma_{1}^{2}}{2}$. In the critical case, namely, if $b=0$, they showed weak consistency of the MLE of $(a, b, \alpha, \beta)$ and determined the asymptotic behavior of the MLE under the additional assumption $a>\frac{\sigma_{1}^{2}}{2}$. In a special supercritical case, namely, when $b<0$, they showed strong consistency of the MLE of $b$, weak consistency of the MLE of $\beta$ and proved asymptotic mixed normality of the MLE of $(a, b, \alpha, \beta)$. Barczy at al. [3, 4] studied the asymptotic behavior of maximum likelihood estimators for a jump-type Heston model and for the growth rate of a jump-type CIR process, respectively, based on continuous time observations.

We consider general two-factor affine diffusions (1.1). In the subcritical case, i.e., when $b>0$ and $\gamma>0$, we prove strong consistency and asymptotic normality of $\left(\widehat{a}_{T}, \widehat{b}_{T}, \widehat{\alpha}_{T}, \widehat{\beta}_{T}, \widehat{\gamma}_{T}\right)$ under the additional assumptions $a>0, \sigma_{1}>0$ and $\left(1-\varrho^{2}\right) \sigma_{2}^{2}+\sigma_{3}^{2}>0$. In a special critical case, namely if $b=0$ and $\gamma=0$, we show weak consistency of $\left(\widehat{b}_{T}, \widehat{\beta}_{T}, \widehat{\gamma}_{T}\right)$ and determine the asymptotic behavior of $\left(\widehat{a}_{T}, \widehat{b}_{T}, \widehat{\alpha}_{T}, \widehat{\beta}_{T}, \widehat{\gamma}_{T}\right)$ under the additional assumptions $\beta=0$ and $\left(1-\varrho^{2}\right) \sigma_{2}^{2}+\sigma_{3}^{2}>0$. In a special supercritical case, namely, when $\gamma<b<0$, we show strong consistency of $\widehat{b}_{T}$, weak consistency of $\left(\widehat{\beta}_{T}, \widehat{\gamma}_{T}\right)$ and prove asymptotic mixed normality of $\left(\widehat{a}_{T}, \widehat{b}_{T}, \widehat{\alpha}_{T}, \widehat{\beta}_{T}, \widehat{\gamma}_{T}\right)$ under the additional assumptions $\alpha \beta \leqslant 0, \sigma_{1}>0$, and either $\sigma_{3}>0$, or $\left(a-\frac{\sigma_{1}^{2}}{2}\right)\left(1-\varrho^{2}\right) \sigma_{2}^{2}>0$. Note that we decided to deal with the CLSE of $(a, b, \alpha, \beta, \gamma)$, since the MLE of $(a, b, \alpha, \beta, \gamma)$ contains, for example, $\int_{0}^{T} \frac{X_{t}}{\left(1-\varrho^{2}\right) \sigma_{2}^{2} Y_{t}+\sigma_{3}^{2}} \mathrm{~d} t$, and the question of the asymptotic behavior of this integral as $T \rightarrow \infty$ is still open in the critical and supercritical cases. 


\section{The affine two-factor model}

Let $\mathbb{N}, \mathbb{Z}_{+}, \mathbb{R}, \mathbb{R}_{+}, \mathbb{R}_{++}, \mathbb{R}_{-}, \mathbb{R}_{--}$and $\mathbb{C}$ denote the sets of positive integers, non-negative integers, real numbers, non-negative real numbers, positive real numbers, non-positive real numbers, negative real numbers and complex numbers, respectively. For $x, y \in \mathbb{R}$, we will use the notations $x \wedge y:=\min (x, y)$ and $x \vee y:=\max (x, y)$. By $C_{\mathrm{c}}^{2}\left(\mathbb{R}_{+} \times \mathbb{R}, \mathbb{R}\right)$, we denote the set of twice continuously differentiable real-valued functions on $\mathbb{R}_{+} \times \mathbb{R}$ with compact support. Let $(\Omega, \mathcal{F}, \mathbb{P})$ be a probability space equipped with the augmented filtration $\left(\mathcal{F}_{t}\right)_{t \in \mathbb{R}_{+}}$corresponding to $\left(W_{t}, B_{t}, L_{t}\right)_{t \in \mathbb{R}_{+}}$and a given initial value $\left(\eta_{0}, \xi_{0}\right)$ being independent of $\left(W_{t}, B_{t}, L_{t}\right)_{t \in \mathbb{R}_{+}}$such that $\mathbb{P}\left(\eta_{0} \in \mathbb{R}_{+}\right)=1$, constructed as in Karatzas and Shreve [24, Section 5.2]. Note that $\left(\mathcal{F}_{t}\right)_{t \in \mathbb{R}_{+}}$satisfies the usual conditions, i.e., the filtration $\left(\mathcal{F}_{t}\right)_{t \in \mathbb{R}_{+}}$is right-continuous and $\mathcal{F}_{0}$ contains all the $\mathbb{P}$-null sets in $\mathcal{F}$. We will denote the convergence in distribution, convergence in probability, almost surely convergence and equality in distribution by $\stackrel{\mathcal{D}}{\longrightarrow}, \stackrel{\mathbb{P}}{\longrightarrow}, \stackrel{\text { a.s. }}{\longrightarrow}$ and $\stackrel{\mathcal{D}}{=}$, respectively. By $\|\boldsymbol{x}\|$ and $\|\boldsymbol{A}\|$, we denote the Euclidean norm of a vector $\boldsymbol{x} \in \mathbb{R}^{d}$ and the induced matrix norm of a matrix $\boldsymbol{A} \in \mathbb{R}^{d \times d}$, respectively. By $\boldsymbol{I}_{d} \in \mathbb{R}^{d \times d}$, we denote the $d \times d$ unit matrix. For quadratic matrices $\boldsymbol{A}_{1}, \ldots, \boldsymbol{A}_{k}, \operatorname{diag}\left(\boldsymbol{A}_{1}, \ldots, \boldsymbol{A}_{k}\right)$ will denote the quadratic block matrix containing the matrices $\boldsymbol{A}_{1}, \ldots, \boldsymbol{A}_{k}$ in its diagonal.

The next proposition is about the existence and uniqueness of a strong solution of the SDE (1.1), see Bolyog and Pap [11, Proposition 2.2].

2.1 Proposition. Let $\left(\eta_{0}, \xi_{0}\right)$ be a random vector independent of the process $\left(W_{t}, B_{t}, L_{t}\right)_{t \in \mathbb{R}_{+}}$satisfying $\mathbb{P}\left(\eta_{0} \in \mathbb{R}_{+}\right)=1$. Then for all $a \in \mathbb{R}_{+}, \quad b, \alpha, \beta, \gamma \in \mathbb{R}, \sigma_{1}, \sigma_{2}, \sigma_{3} \in \mathbb{R}_{+}, \varrho \in[-1,1]$, there is $a$ (pathwise) unique strong solution $\left(Y_{t}, X_{t}\right)_{t \in \mathbb{R}_{+}}$of the $S D E$ (1.1) such that $\mathbb{P}\left(\left(Y_{0}, X_{0}\right)=\left(\eta_{0}, \xi_{0}\right)\right)=1$ and $\mathbb{P}\left(Y_{t} \in \mathbb{R}_{+}\right.$for all $\left.t \in \mathbb{R}_{+}\right)=1$. Further, for all $s, t \in \mathbb{R}_{+}$with $s \leqslant t$, we have

$$
Y_{t}=\mathrm{e}^{-b(t-s)} Y_{s}+a \int_{s}^{t} \mathrm{e}^{-b(t-u)} \mathrm{d} u+\sigma_{1} \int_{s}^{t} \mathrm{e}^{-b(t-u)} \sqrt{Y_{u}} \mathrm{~d} W_{u}
$$

and

$$
\begin{aligned}
X_{t}= & \mathrm{e}^{-\gamma(t-s)} X_{s}+\int_{s}^{t} \mathrm{e}^{-\gamma(t-u)}\left(\alpha-\beta Y_{u}\right) \mathrm{d} u \\
& +\sigma_{2} \int_{s}^{t} \mathrm{e}^{-\gamma(t-u)} \sqrt{Y_{u}}\left(\varrho \mathrm{d} W_{u}+\sqrt{1-\varrho^{2}} \mathrm{~d} B_{u}\right)+\sigma_{3} \int_{s}^{t} \mathrm{e}^{-\gamma(t-u)} \mathrm{d} L_{u} .
\end{aligned}
$$

Moreover, $\left(Y_{t}, X_{t}\right)_{t \in \mathbb{R}_{+}}$is a two-factor affine process with infinitesimal generator

$$
\begin{aligned}
\left(\mathcal{A}_{(Y, X)} f\right)(y, x)= & (a-b y) f_{1}^{\prime}(y, x)+(\alpha-\beta y-\gamma x) f_{2}^{\prime}(y, x) \\
& +\frac{1}{2} y\left[\sigma_{1}^{2} f_{1,1}^{\prime \prime}(y, x)+2 \varrho \sigma_{1} \sigma_{2} f_{1,2}^{\prime \prime}(y, x)+\sigma_{2}^{2} f_{2,2}^{\prime \prime}(y, x)\right]+\frac{1}{2} \sigma_{3}^{2} f_{2,2}^{\prime \prime}(y, x),
\end{aligned}
$$

where $(y, x) \in \mathbb{R}_{+} \times \mathbb{R}, \quad f \in \mathcal{C}_{c}^{2}\left(\mathbb{R}_{+} \times \mathbb{R}, \mathbb{R}\right)$, and $f_{i}^{\prime}, i \in\{1,2\}$, and $f_{i, j}^{\prime \prime}, \quad i, j \in\{1,2\}$, denote the first and second order partial derivatives of $f$ with respect to its $i$-th and $i$-th and $j$-th variables.

Conversely, every two-factor affine diffusion process is a (pathwise) unique strong solution of a $S D E$ (1.1) with suitable parameters $a \in \mathbb{R}_{+}, \quad b, \alpha, \beta, \gamma \in \mathbb{R}, \sigma_{1}, \sigma_{2}, \sigma_{3} \in \mathbb{R}_{+}$and $\varrho \in[-1,1]$.

The next proposition gives the asymptotic behavior of the first moment of the process $\left(Y_{t}, X_{t}\right)_{t \in \mathbb{R}_{+}}$ as $t \rightarrow \infty$, see Bolyog and Pap [11, Proposition 2.3]. 
2.2 Proposition. Let us consider the two-factor affine diffusion model (1.1) with $a \in \mathbb{R}_{+}, b, \alpha, \beta, \gamma \in$ $\mathbb{R}, \quad \sigma_{1}, \sigma_{2}, \sigma_{3} \in \mathbb{R}_{+}, \varrho \in[-1,1]$. Suppose that $\mathbb{E}\left(Y_{0}\left|X_{0}\right|\right)<\infty$. In case of $b \in \mathbb{R}_{++}$we have $\mathbb{E}\left(Y_{t}\right)=\frac{a}{b}+\mathrm{O}\left(\mathrm{e}^{-b t}\right)$ and

$$
\mathbb{E}\left(X_{t}\right)= \begin{cases}\frac{\alpha}{\gamma}-\frac{a \beta}{b \gamma}+\mathrm{O}\left(\mathrm{e}^{-(b \wedge \gamma) t}\right), & \gamma \in \mathbb{R}_{++}, \\ \left(\alpha-\frac{a \beta}{b}\right) t+\mathrm{O}(1), & \gamma=0, \\ \left(\frac{\beta}{\gamma-b} \mathbb{E}\left(Y_{0}\right)+\mathbb{E}\left(X_{0}\right)-\frac{\alpha}{\gamma}+\frac{a \beta}{b \gamma}-\frac{a \beta}{(\gamma-b) b}\right) \mathrm{e}^{-\gamma t}+\mathrm{O}(1), & \gamma \in \mathbb{R}_{--.} .\end{cases}
$$

In case of $b=0$ we have $\mathbb{E}\left(Y_{t}\right)=a t+\mathrm{O}(1)$ and

$$
\mathbb{E}\left(X_{t}\right)= \begin{cases}-\frac{a \beta}{\gamma} t+\mathrm{O}(1), & \gamma \in \mathbb{R}_{++}, \\ -\frac{1}{2} a \beta t^{2}+\mathrm{O}(t), & \gamma=0, \\ \left(\frac{\beta}{\gamma} \mathbb{E}\left(Y_{0}\right)+\mathbb{E}\left(X_{0}\right)-\frac{\alpha}{\gamma}-\frac{a \beta}{\gamma^{2}}\right) \mathrm{e}^{-\gamma t}+\mathrm{O}(t), & \gamma \in \mathbb{R}_{--} .\end{cases}
$$

In case of $b \in \mathbb{R}_{--}$we have $\mathbb{E}\left(Y_{t}\right)=\left(\mathbb{E}\left(Y_{0}\right)-\frac{a}{b}\right) \mathrm{e}^{-b t}+\mathrm{O}(1)$ and

$$
\mathbb{E}\left(X_{t}\right)= \begin{cases}\left(-\frac{\beta}{\gamma-b} \mathbb{E}\left(Y_{0}\right)+\frac{a \beta}{(\gamma-b) b}\right) \mathrm{e}^{-b t}+\mathrm{O}(1), & \gamma \in \mathbb{R}_{++}, \\ \left(\frac{\beta}{b} \mathbb{E}\left(Y_{0}\right)+\mathbb{E}\left(X_{0}\right)-\frac{\beta a}{b^{2}}\right) \mathrm{e}^{-b t}+\mathrm{O}(t), & \gamma=0, \\ \left(-\frac{\beta}{\gamma-b} \mathbb{E}\left(Y_{0}\right)+\frac{a \beta}{(\gamma-b) b}\right) \mathrm{e}^{-b t}+\mathrm{O}\left(\mathrm{e}^{-\gamma t}\right), & \gamma \in(b, 0), \\ \left(-\beta \mathbb{E}\left(Y_{0}\right)+\frac{a \beta}{b}\right) t \mathrm{e}^{-b t}+\mathrm{O}\left(\mathrm{e}^{-\gamma t}\right), & \gamma=b, \\ \left(\frac{\beta}{\gamma-b} \mathbb{E}\left(Y_{0}\right)+\mathbb{E}\left(X_{0}\right)-\frac{\alpha}{\gamma}+\frac{a \beta}{b \gamma}-\frac{a \beta}{b(\gamma-b)}\right) \mathrm{e}^{-\gamma t}+\mathrm{O}\left(\mathrm{e}^{-b t}\right), & \gamma \in(-\infty, b) .\end{cases}
$$

Based on the asymptotic behavior of the first moment of the process $\left(Y_{t}, X_{t}\right)_{t \in \mathbb{R}_{+}}$as $t \rightarrow \infty$, we can classify two-factor affine diffusions in the following way.

2.3 Definition. Let $\left(Y_{t}, X_{t}\right)_{t \in \mathbb{R}_{+}}$be the unique strong solution of the SDE (1.1) satisfying $\mathbb{P}\left(Y_{0} \in\right.$ $\left.\mathbb{R}_{+}\right)=1$. We call $\left(Y_{t}, X_{t}\right)_{t \in \mathbb{R}_{+}}$subcritical, critical or supercritical if $b \wedge \gamma \in \mathbb{R}_{++}, b \wedge \gamma=0$ or $b \wedge \gamma \in \mathbb{R}_{--}$, respectively.

\section{CLSE based on continuous time observations}

Overbeck and Rydén [27] investigated the CIR process $Y$, and for each $T \in \mathbb{R}_{++}$, they defined a $\operatorname{CLSE}\left(\widehat{a}_{T}, \widehat{b}_{T}\right)$ of $(a, b)$ based on continuous time observations $\left(Y_{t}\right)_{t \in[0, T]}$ as the limit in probability of the CLSE $\left(\widehat{a}_{T, n}, \widehat{b}_{T, n}\right)$ of $(a, b)$ based on discrete time observations $\left(Y_{\frac{i T}{n}}\right)_{i \in\{0,1, \ldots, n\}}$ as $n \rightarrow \infty$.

We consider a two-factor affine diffusion process $\left(Y_{t}, X_{t}\right)_{t \in \mathbb{R}_{+}}$given in (1.1) with known $\sigma_{1} \in \mathbb{R}_{++}$, $\sigma_{2}, \sigma_{3} \in \mathbb{R}_{+}$and $\varrho \in[-1,1]$, and with a random initial value $\left(\eta_{0}, \zeta_{0}\right)$ independent of $\left(W_{t}, B_{t}, L_{t}\right)_{t \in \mathbb{R}_{+}}$ satisfying $\mathbb{P}\left(\eta_{0} \in \mathbb{R}_{+}\right)=1$, and we will consider $\boldsymbol{\theta}=(a, b, \alpha, \beta, \gamma)^{\top} \in \mathbb{R}_{+} \times \mathbb{R}^{4}$ as a parameter. The aim of the following discussion is to construct a CLSE of $\boldsymbol{\theta}$ based on continuous time observations $\left(Y_{t}, X_{t}\right)_{t \in[0, T]}$ with some $T \in \mathbb{R}_{++}$. 
Let us recall the CLSE $\widehat{\boldsymbol{\theta}}_{T, n}$ of $\boldsymbol{\theta}$ based on discrete time observations $\left(Y_{\frac{i}{n}}, X_{\frac{i}{n}}\right)_{i \in\{0,1, \ldots,\lfloor n T\rfloor\}}$ with some $n \in \mathbb{N}$, which can be obtained by solving the extremum problem

$$
\widehat{\boldsymbol{\theta}}_{T, n}:=\underset{\boldsymbol{\theta} \in \mathbb{R}^{5}}{\arg \min } \sum_{i=1}^{\lfloor n T\rfloor}\left[\left(Y_{\frac{i}{n}}-\mathbb{E}\left(Y_{\frac{i}{n}} \mid \mathcal{F}_{\frac{i-1}{n}}\right)\right)^{2}+\left(X_{\frac{i}{n}}-\mathbb{E}\left(X_{\frac{i}{n}} \mid \mathcal{F}_{\frac{i-1}{n}}\right)\right)^{2}\right] .
$$

By (2.1) and (2.2), together with Proposition 3.2.10 in Karatzas and Shreve [24], for all $s, t \in \mathbb{R}_{+}$ with $s \leqslant t$, we obtain

$$
\begin{gathered}
\mathbb{E}\left(Y_{t} \mid \mathcal{F}_{s}\right)=\mathrm{e}^{-b(t-s)} Y_{s}+a \int_{s}^{t} \mathrm{e}^{-b(t-u)} \mathrm{d} u \\
\mathbb{E}\left(X_{t} \mid \mathcal{F}_{s}\right)=\mathrm{e}^{-\gamma(t-s)} X_{s}+\alpha \int_{s}^{t} \mathrm{e}^{-\gamma(t-u)} \mathrm{d} u-\beta Y_{s} \int_{s}^{t} \mathrm{e}^{-\gamma(t-u)-b(u-s)} \mathrm{d} u \\
-a \beta \int_{s}^{t} \mathrm{e}^{-\gamma(t-u)}\left(\int_{s}^{u} \mathrm{e}^{-b(u-v)} \mathrm{d} v\right) \mathrm{d} u
\end{gathered}
$$

Thus, for all $i \in \mathbb{N}$, we have

$$
\mathbb{E}\left(Y_{\frac{i}{n}} \mid \mathcal{F}_{\frac{i-1}{n}}\right)=\mathrm{e}^{-\frac{b}{n}} Y_{\frac{i-1}{n}}+a \int_{0}^{\frac{1}{n}} \mathrm{e}^{-b w} \mathrm{~d} w
$$

and

$$
\begin{aligned}
\mathbb{E}\left(X_{\frac{i}{n}} \mid \mathcal{F}_{\frac{i-1}{n}}\right)= & \mathrm{e}^{-\frac{\gamma}{n}} X_{\frac{i-1}{n}}+\alpha \int_{0}^{\frac{1}{n}} \mathrm{e}^{-\gamma w} \mathrm{~d} w-\beta Y_{\frac{i-1}{n}} \int_{0}^{\frac{1}{n}} \mathrm{e}^{(\gamma-b) w-\frac{\gamma}{n}} \mathrm{~d} w \\
& -a \beta \int_{0}^{\frac{1}{n}} \mathrm{e}^{\gamma w-\frac{\gamma}{n}}\left(\int_{0}^{w} \mathrm{e}^{-b(w-v)} \mathrm{d} v\right) \mathrm{d} w
\end{aligned}
$$

Consequently,

$$
\begin{aligned}
\widehat{\boldsymbol{\theta}}_{T, n}=\underset{(a, b, \alpha, \beta, \gamma)^{\top} \in \mathbb{R}^{5}}{\arg \min } \sum_{i=1}^{\lfloor n T\rfloor}[ & \left(Y_{\frac{i}{n}}-Y_{\frac{i-1}{n}}-\left(c-d Y_{\frac{i-1}{n}}\right)\right)^{2} \\
& \left.+\left(X_{\frac{i}{n}}-X_{\frac{i-1}{n}}-\left(\delta-\varepsilon Y_{\frac{i-1}{n}}-\zeta X_{\frac{i-1}{n}}\right)\right)^{2}\right]
\end{aligned}
$$

where

$$
(c, d, \delta, \varepsilon, \zeta):=\left(c_{n}(a, b), d_{n}(b), \delta_{n}(a, b, \alpha, \beta, \gamma), \varepsilon_{n}(b, \beta, \gamma), \zeta_{n}(\gamma)\right):=g_{n}(a, b, \alpha, \beta, \gamma)
$$

with

$$
\begin{gathered}
c:=c_{n}(a, b):=a \int_{0}^{\frac{1}{n}} \mathrm{e}^{-b w} \mathrm{~d} w, \quad d:=d_{n}(b):=1-\mathrm{e}^{-\frac{b}{n}} \\
\delta:=\delta_{n}(a, b, \alpha, \beta, \gamma):=\alpha \int_{0}^{\frac{1}{n}} \mathrm{e}^{-\gamma w} \mathrm{~d} w-a \beta \int_{0}^{\frac{1}{n}} \mathrm{e}^{\gamma w-\frac{\gamma}{n}}\left(\int_{0}^{w} \mathrm{e}^{-b(w-v)} \mathrm{d} v\right) \mathrm{d} w \\
\varepsilon:=\varepsilon_{n}(b, \beta, \gamma):=\beta \int_{0}^{\frac{1}{n}} \mathrm{e}^{(\gamma-b) w-\frac{\gamma}{n}} \mathrm{~d} w, \quad \zeta:=\zeta_{n}(\gamma):=1-\mathrm{e}^{-\frac{\gamma}{n}}
\end{gathered}
$$


The function $g_{n}: \mathbb{R}^{5} \rightarrow \mathbb{R} \times(-\infty, 1) \times \mathbb{R}^{2} \times(-\infty, 1)$ is bijective, so first we determine the CLSE $\left(\widehat{c}_{T, n}, \widehat{d}_{T, n}, \widehat{\delta}_{T, n}, \widehat{\varepsilon}_{T, n}, \widehat{\zeta}_{T, n}\right)$ of the transformed parameters $(c, d, \delta, \varepsilon, \zeta)$ by minimizing the sum on the right-hand side of (3.3) with respect to $(c, d, \delta, \varepsilon, \zeta)$. We have

$$
\begin{aligned}
\left(\widehat{c}_{T, n}, \widehat{d}_{T, n}\right) & =\underset{(c, d)^{\top} \in \mathbb{R}^{2}}{\arg \min } \sum_{i=1}^{\lfloor n T\rfloor}\left(Y_{\frac{i}{n}}-Y_{\frac{i-1}{n}}-\left(c-d Y_{\frac{i-1}{n}}\right)\right)^{2}, \\
\left(\widehat{\delta}_{T, n}, \widehat{\varepsilon}_{T, n}, \widehat{\zeta}_{T, n}\right) & =\underset{(\delta, \varepsilon, \zeta)^{\top} \in \mathbb{R}^{3}}{\arg \min } \sum_{i=1}^{\lfloor n T\rfloor}\left(X_{\frac{i}{n}}-X_{\frac{i-1}{n}}-\left(\delta-\varepsilon Y_{\frac{i-1}{n}}-\zeta X_{\frac{i-1}{n}}\right)\right)^{2},
\end{aligned}
$$

hence, similarly as on page 675 in Barczy et al. [5], we get

$$
\left[\begin{array}{c}
\widehat{c}_{T, n} \\
\widehat{d}_{T, n}
\end{array}\right]=\left(\boldsymbol{\Gamma}_{T, n}^{(1)}\right)^{-1} \boldsymbol{\varphi}_{T, n}^{(1)}, \quad\left[\begin{array}{c}
\widehat{\delta}_{T, n} \\
\widehat{\varepsilon}_{T, n} \\
\widehat{\zeta}_{T, n}
\end{array}\right]=\left(\boldsymbol{\Gamma}_{T, n}^{(2)}\right)^{-1} \boldsymbol{\varphi}_{T, n}^{(2)}
$$

with

$$
\begin{aligned}
& \boldsymbol{\Gamma}_{T, n}^{(1)}:=\left[\begin{array}{cc}
\lfloor n T\rfloor & -\sum_{i=1}^{\lfloor n T\rfloor} Y_{\frac{i-1}{n}} \\
-\sum_{i=1}^{\lfloor n T\rfloor} Y_{\frac{i-1}{n}} & \sum_{i=1}^{\lfloor n T\rfloor} Y_{\frac{i-1}{n}}^{2}
\end{array}\right], \quad \boldsymbol{\varphi}_{T, n}^{(1)}:=\left[\begin{array}{c}
Y_{\frac{\lfloor n T\rfloor}{n}}-Y_{0} \\
-\sum_{i=1}^{\lfloor n T\rfloor}\left(Y_{\frac{i}{n}}-Y_{\frac{i-1}{n}}\right) Y_{\frac{i-1}{n}}
\end{array}\right], \\
& \boldsymbol{\Gamma}_{T, n}^{(2)}:=\left[\begin{array}{ccc}
\lfloor n T\rfloor & -\sum_{i=1}^{\lfloor n T\rfloor} Y_{\frac{i-1}{n}} & -\sum_{i=1}^{\lfloor n T\rfloor} X_{\frac{i-1}{n}} \\
-\sum_{i=1}^{\lfloor n T\rfloor} Y_{\frac{i-1}{n}} & \sum_{i=1}^{\lfloor n T\rfloor} Y_{\frac{i-1}{n}}^{2} & \sum_{i=1}^{\lfloor n T\rfloor} Y_{\frac{i-1}{n}} X_{\frac{i-1}{n}} \\
-\sum_{i=1}^{\lfloor n T\rfloor} X_{\frac{i-1}{n}} & \sum_{i=1}^{\lfloor n T\rfloor} Y_{\frac{i-1}{n}} X_{\frac{i-1}{n}} & \sum_{i=1}^{\lfloor n T\rfloor} X_{\frac{i-1}{n}}^{2}
\end{array}\right], \quad \boldsymbol{\varphi}_{T, n}^{(2)}:=\left[\begin{array}{c}
X_{\frac{\lfloor n T\rfloor}{n}}-X_{0} \\
-\sum_{i=1}^{\lfloor n T\rfloor}\left(X_{\frac{i}{n}}-X_{\frac{i-1}{n}}\right) Y_{\frac{i-1}{n}} \\
-\sum_{i=1}^{\lfloor n T\rfloor}\left(X_{\frac{i}{n}}-X_{\frac{i-1}{n}}\right) X_{\frac{i-1}{n}}
\end{array}\right]
\end{aligned}
$$

on the event where the random matrices $\boldsymbol{\Gamma}_{T, n}^{(1)}$ and $\boldsymbol{\Gamma}_{T, n}^{(2)}$ are invertible.

3.1 Lemma. Let us consider the two-factor affine diffusion model (1.1) with $a \in \mathbb{R}_{+}, b, \alpha, \beta, \gamma \in \mathbb{R}$, $\sigma_{1} \in \mathbb{R}_{++}, \quad \sigma_{2}, \sigma_{3} \in \mathbb{R}_{+}$and $\varrho \in[-1,1]$ with a random initial value $\left(\eta_{0}, \zeta_{0}\right)$ independent of $\left(W_{t}, B_{t}, L_{t}\right)_{t \in \mathbb{R}_{+}}$satisfying $\mathbb{P}\left(\eta_{0} \in \mathbb{R}_{+}\right)=1$. Suppose that $\left(1-\varrho^{2}\right) \sigma_{2}^{2}+\sigma_{3}^{2}>0$. Then for each $T \in \mathbb{R}_{++}$and $n \in \mathbb{N}$, the random matrices $\Gamma_{T, n}^{(1)}$ and $\boldsymbol{\Gamma}_{T, n}^{(2)}$ are invertible almost surely, and hence there exists a unique CLSE $\left(\widehat{c}_{T, n}, \widehat{d}_{T, n}, \widehat{\delta}_{T, n}, \widehat{\varepsilon}_{T, n}, \widehat{\zeta}_{T, n}\right)$ of $(c, d, \delta, \varepsilon, \zeta)$ taking the form given in (3.5).

Proof. The aim of the following discussion is to show that the random matrix $\boldsymbol{\Gamma}_{T, n}^{(1)}$ is almost surely strictly positive definite checking that for all $\boldsymbol{x} \in \mathbb{R}^{2} \backslash\{\mathbf{0}\}$, we have $\boldsymbol{x}^{\top} \boldsymbol{\Gamma}_{T, n}^{(1)} \boldsymbol{x}>0$ almost surely. Indeed, for all $\boldsymbol{x}=\left(x_{1}, x_{2}\right)^{\top} \in \mathbb{R}^{2}$,

$$
\left[\begin{array}{l}
x_{1} \\
x_{2}
\end{array}\right]^{\top} \boldsymbol{\Gamma}_{T, n}^{(1)}\left[\begin{array}{l}
x_{1} \\
x_{2}
\end{array}\right]=\sum_{i=1}^{\lfloor n T\rfloor}\left[\begin{array}{l}
x_{1} \\
x_{2}
\end{array}\right]^{\top}\left[\begin{array}{c}
1 \\
-Y_{\frac{i-1}{n}}
\end{array}\right]\left[\begin{array}{c}
1 \\
-Y_{\frac{i-1}{n}}
\end{array}\right]^{\top}\left[\begin{array}{l}
x_{1} \\
x_{2}
\end{array}\right] \mathrm{d} s=\sum_{i=1}^{\lfloor n T\rfloor}\left(x_{1}-x_{2} Y_{\frac{i-1}{n}}\right)^{2} \mathrm{~d} s \geqslant 0
$$


and $\boldsymbol{x}^{\top} \boldsymbol{\Gamma}_{T, n}^{(1)} \boldsymbol{x}=0$ if and only if $x_{1}-x_{2} Y_{\frac{i-1}{n}}=0$ for all $i \in\{0,1, \ldots,\lfloor n T\rfloor\}$, which happens with probability 0 , since $\boldsymbol{x}=\left(x_{1}, x_{2}\right)^{\top} \neq \mathbf{0}$ and, for each $i \in\{1, \ldots,\lfloor n T\rfloor\}$, the distribution of $Y_{\frac{i-1}{n}}$ is absolutely continuous, since the conditional distribution of $Y_{\frac{i-1}{n}}$ given $Y_{0}$ is absolutely continuous, see, eg., Ben Alaya and Kebaier [10, Proof of Proposition 2] and Ikeda and Watanabe [22, page 222].

In a similar way, the stochastic matrix $\Gamma_{T, n}^{(2)}$ is almost surely strictly positive definite, since for all $\boldsymbol{x} \in \mathbb{R}^{3} \backslash\{\mathbf{0}\}$, we have $\boldsymbol{x}^{\top} \boldsymbol{\Gamma}_{T, n}^{(2)} \boldsymbol{x}>0$ almost surely. Indeed, for all $\boldsymbol{x}=\left(x_{1}, x_{2}, x_{3}\right)^{\top} \in \mathbb{R}^{3}$,

$$
\left[\begin{array}{l}
x_{1} \\
x_{2} \\
x_{3}
\end{array}\right]^{\top} \boldsymbol{\Gamma}_{T, n}^{(2)}\left[\begin{array}{l}
x_{1} \\
x_{2} \\
x_{3}
\end{array}\right]=\sum_{i=1}^{\lfloor n T\rfloor}\left[\begin{array}{l}
x_{1} \\
x_{2} \\
x_{3}
\end{array}\right]^{\top}\left[\begin{array}{c}
1 \\
-Y_{\frac{i-1}{n}} \\
-X_{\frac{i-1}{n}}
\end{array}\right]\left[\begin{array}{c}
1 \\
-Y_{\frac{i-1}{n}} \\
-X_{\frac{i-1}{n}}
\end{array}\right]^{\top}\left[\begin{array}{l}
x_{1} \\
x_{2} \\
x_{3}
\end{array}\right] \mathrm{d} s=\sum_{i=1}^{\lfloor n T\rfloor}\left(x_{1}-x_{2} Y_{\frac{i-1}{n}}-x_{3} X_{\frac{i-1}{n}}\right)^{2} \mathrm{~d} s \geqslant 0
$$

and $\boldsymbol{x}^{\top} \boldsymbol{\Gamma}_{T, n}^{(2)} \boldsymbol{x}=0$ if and only if $x_{1}-x_{2} Y_{\frac{i-1}{n}}-x_{3} X_{\frac{i-1}{n}}=0$ for all $i \in\{0,1, \ldots,\lfloor n T\rfloor\}$, which happens with probability 0 , since $\boldsymbol{x}=\left(x_{1}, x_{2}, x_{3}\right)^{\top} \neq \mathbf{0}$ and, for each $i \in\{1, \ldots,\lfloor n T\rfloor\}$, the distribution of $\left(Y_{\frac{i-1}{n}}, X_{\frac{i-1}{n}}\right)$ is absolutely continuous, because, as in the proof of part (b) in the proof of Theorem A.1 in Bolyog and Pap [11, the conditional distribution of $\left(Y_{\frac{i-1}{n}}, X_{\frac{i-1}{n}}\right)$ given $\left(Y_{0}, X_{0}\right)$ is absolutely continuous.

3.2 Remark. The first order Taylor approximation of $g_{n}(a, b, \alpha, \beta, \gamma)$ at $(0,0,0,0,0)$ is $\frac{1}{n}(a, b, \alpha, \beta, \gamma)$, hence we obtain the first order Taylor approximations

$$
\begin{gathered}
Y_{\frac{i}{n}}-\mathbb{E}\left(Y_{\frac{i}{n}} \mid \mathcal{F}_{\frac{i-1}{n}}\right) \approx Y_{\frac{i}{n}}-Y_{\frac{i-1}{n}}-\frac{1}{n}\left(a-b Y_{\frac{i-1}{n}}\right), \\
X_{\frac{i}{n}}-\mathbb{E}\left(X_{\frac{i}{n}} \mid \mathcal{F}_{\frac{i-1}{n}}\right) \approx X_{\frac{i}{n}}-X_{\frac{i-1}{n}}-\frac{1}{n}\left(\alpha-\beta Y_{\frac{i-1}{n}}-\gamma X_{\frac{i-1}{n}}\right) .
\end{gathered}
$$

Using these approximations, one can define an approximate CLSE $\widehat{\boldsymbol{\theta}}_{T, n}^{\text {approx }}$ of $\boldsymbol{\theta}$ based on discrete

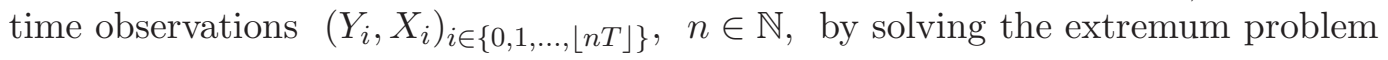

$$
\begin{aligned}
\widehat{\boldsymbol{\theta}}_{T, n}^{\mathrm{approx}}:=\underset{(a, b, \alpha, \beta, \gamma)^{\top} \in \mathbb{R}^{5}}{\arg \min } \sum_{i=1}^{\lfloor n T\rfloor} & {\left[\left(Y_{\frac{i}{n}}-Y_{\frac{i-1}{n}}-\frac{1}{n}\left(a-b Y_{\frac{i-1}{n}}\right)\right)^{2}\right.} \\
& \left.+\left(X_{\frac{i}{n}}-X_{\frac{i-1}{n}}-\frac{1}{n}\left(\alpha-\beta Y_{\frac{i-1}{n}}-\gamma X_{\frac{i-1}{n}}\right)\right)^{2}\right],
\end{aligned}
$$

hence $\widehat{\boldsymbol{\theta}}_{T, n}^{\text {approx }}=n\left(\widehat{c}_{T, n}, \widehat{d}_{T, n}, \widehat{\delta}_{T, n}, \widehat{\varepsilon}_{T, n}, \widehat{\zeta}_{T, n}\right)^{\top}$. This definition of approximate CLSE can be considered as the definition of LSE given in Hu and Long [20, formula (1.2)] for generalized Ornstein-Uhlenbeck processes driven by $\alpha$-stable motions, see also $\mathrm{Hu}$ and Long [21, formula (3.1)]. For a heuristic motivation of the estimator $\widehat{\boldsymbol{\theta}}_{n}^{\text {approx }}$ based on discrete observations, see, e.g., Hu and Long [19, page 178] (formulated for Langevin equations). 
We have

$$
\begin{aligned}
& \frac{1}{n} \Gamma_{T, n}^{(1)} \stackrel{\text { a.s. }}{\longrightarrow}\left[\begin{array}{cc}
T & -\int_{0}^{T} Y_{s} \mathrm{~d} s \\
-\int_{0}^{T} Y_{s} \mathrm{~d} s & \int_{0}^{T} Y_{s}^{2} \mathrm{~d} s
\end{array}\right]=: \boldsymbol{G}_{T}^{(1)}, \\
& \frac{1}{n} \boldsymbol{\Gamma}_{T, n}^{(2)} \stackrel{\text { a.s. }}{\longrightarrow}\left[\begin{array}{ccc}
T & -\int_{0}^{T} Y_{s} \mathrm{~d} s & -\int_{0}^{T} X_{s} \mathrm{~d} s \\
-\int_{0}^{T} Y_{s} \mathrm{~d} s & \int_{0}^{T} Y_{s}^{2} \mathrm{~d} s & \int_{0}^{T} X_{s} Y_{s} \mathrm{~d} s \\
-\int_{0}^{T} X_{s} \mathrm{~d} s & \int_{0}^{T} X_{s} Y_{s} \mathrm{~d} s & \int_{0}^{T} X_{s}^{2} \mathrm{~d} s
\end{array}\right]=: \boldsymbol{G}_{T}^{(2)}
\end{aligned}
$$

as $n \rightarrow \infty$, since $\left(Y_{t}, X_{t}\right)_{t \in \mathbb{R}_{+}}$is almost surely continuous. By Proposition I.4.44 in Jacod and Shiryaev [23] with the Riemann sequence of deterministic subdivisions $\left(\frac{i}{n} \wedge T\right)_{i \in \mathbb{N}}, \quad n \in \mathbb{N}$., we obtain

$$
\boldsymbol{\varphi}_{T, n}^{(1)} \stackrel{\mathbb{P}}{\longrightarrow}\left[\begin{array}{c}
Y_{T}-Y_{0} \\
-\int_{0}^{T} Y_{s} \mathrm{~d} Y_{s}
\end{array}\right]=: \boldsymbol{f}_{T}^{(1)}, \quad \boldsymbol{\varphi}_{T, n}^{(2)} \stackrel{\mathbb{P}}{\longrightarrow}\left[\begin{array}{c}
X_{T}-X_{0} \\
-\int_{0}^{T} Y_{s} \mathrm{~d} X_{s} \\
-\int_{0}^{T} X_{s} \mathrm{~d} X_{s}
\end{array}\right]=: \boldsymbol{f}_{T}^{(2)},
$$

as $n \rightarrow \infty$. By Slutsky's lemma, using also Lemma 3.1, we conclude

$$
\widehat{\boldsymbol{\theta}}_{T, n}^{\text {approx }}=n\left[\begin{array}{c}
\widehat{c}_{T, n} \\
\widehat{d}_{T, n} \\
\widehat{\delta}_{T, n} \\
\widehat{\varepsilon}_{T, n} \\
\widehat{\zeta}_{T, n}
\end{array}\right] \stackrel{\mathbb{P}}{\longrightarrow}\left[\begin{array}{l}
\left(\boldsymbol{G}_{T}^{(1)}\right)^{-1} \boldsymbol{f}_{T}^{(1)} \\
\left(\boldsymbol{G}_{T}^{(2)}\right)^{-1} \boldsymbol{f}_{T}^{(2)}
\end{array}\right]=:\left[\begin{array}{c}
\widehat{a}_{T} \\
\widehat{b}_{T} \\
\widehat{\alpha}_{T} \\
\widehat{\beta}_{T} \\
\widehat{\gamma}_{T}
\end{array}\right]=: \widehat{\boldsymbol{\theta}}_{T} \quad \text { as } n \rightarrow \infty,
$$

whenever the random matrices $\boldsymbol{G}_{T}^{(1)}$ and $\boldsymbol{G}_{T}^{(2)}$ are invertible.

3.3 Lemma. Let us consider the two-factor affine diffusion model (1.1) with $a \in \mathbb{R}_{+}, b, \alpha, \beta, \gamma \in \mathbb{R}$, $\sigma_{1} \in \mathbb{R}_{++}, \quad \sigma_{2}, \sigma_{3} \in \mathbb{R}_{+}$and $\varrho \in[-1,1]$ with a random initial value $\left(\eta_{0}, \zeta_{0}\right)$ independent of $\left(W_{t}, B_{t}, L_{t}\right)_{t \in \mathbb{R}_{+}}$satisfying $\mathbb{P}\left(\eta_{0} \in \mathbb{R}_{+}\right)=1$. Suppose that $\left(1-\varrho^{2}\right) \sigma_{2}^{2}+\sigma_{3}^{2}>0$. Then for each $T \in \mathbb{R}_{++}$, the random matrices $\boldsymbol{G}_{T}^{(1)}$ and $\boldsymbol{G}_{T}^{(2)}$ are invertible almost surely, and hence $\widehat{\boldsymbol{\theta}}_{T}$ given in (3.6) exists almost surely. Moreover, $\widehat{\boldsymbol{\theta}}_{T, n} \stackrel{\mathbb{P}}{\longrightarrow} \widehat{\boldsymbol{\theta}}_{T}$ as $n \rightarrow \infty$.

Proof. The aim of the following discussion is to show that the random matrix $\boldsymbol{G}_{T}^{(1)}$ is almost surely strictly positive definite checking that for all $\boldsymbol{x} \in \mathbb{R}^{2} \backslash\{\mathbf{0}\}$, we have $\boldsymbol{x}^{\top} \boldsymbol{G}_{T}^{(1)} \boldsymbol{x}>0$ almost surely. Indeed, for all $\boldsymbol{x}=\left(x_{1}, x_{2}\right)^{\top} \in \mathbb{R}^{2}$,

$$
\left[\begin{array}{l}
x_{1} \\
x_{2}
\end{array}\right]^{\top} \boldsymbol{G}_{T}^{(1)}\left[\begin{array}{l}
x_{1} \\
x_{2}
\end{array}\right]=\int_{0}^{T}\left[\begin{array}{l}
x_{1} \\
x_{2}
\end{array}\right]^{\top}\left[\begin{array}{c}
1 \\
-Y_{s}
\end{array}\right]\left[\begin{array}{c}
1 \\
-Y_{s}
\end{array}\right]^{\top}\left[\begin{array}{l}
x_{1} \\
x_{2}
\end{array}\right] \mathrm{d} s=\int_{0}^{T}\left(x_{1}-x_{2} Y_{s}\right)^{2} \mathrm{~d} s \geqslant 0
$$

and $\boldsymbol{x}^{\top} \boldsymbol{G}_{T}^{(1)} \boldsymbol{x}=0$ if and only if $x_{1}-x_{2} Y_{s}=0$ for all $s \in[0, T]$, which happens with probability 0 , since $\boldsymbol{x}=\left(x_{1}, x_{2}\right)^{\top} \neq \mathbf{0}$ and, for each $s \in(0, T]$, the distribution of $Y_{s}$ is absolutely continuous, since the conditional distribution of $Y_{s}$ given $Y_{0}$ is absolutely continuous, see, eg., Ben Alaya and Kebaier [10, Proof of Proposition 2] and Ikeda and Watanabe [22, page 222]. 
In a similar way, the stochastic matrix $\boldsymbol{G}_{T}^{(2)}$ is almost surely strictly positive definite, since for all $\boldsymbol{x} \in \mathbb{R}^{3} \backslash\{\mathbf{0}\}$, we have $\boldsymbol{x}^{\top} \boldsymbol{G}_{T}^{(2)} \boldsymbol{x}>0$ almost surely. Indeed, for all $\boldsymbol{x}=\left(x_{1}, x_{2}, x_{3}\right)^{\top} \in \mathbb{R}^{3}$,

$$
\left[\begin{array}{l}
x_{1} \\
x_{2} \\
x_{3}
\end{array}\right]^{\top} \boldsymbol{G}_{T}^{(2)}\left[\begin{array}{l}
x_{1} \\
x_{2} \\
x_{3}
\end{array}\right]=\int_{0}^{T}\left[\begin{array}{l}
x_{1} \\
x_{2} \\
x_{3}
\end{array}\right]^{\top}\left[\begin{array}{c}
1 \\
-Y_{s} \\
-X_{s}
\end{array}\right]\left[\begin{array}{c}
1 \\
-Y_{s} \\
-X_{s}
\end{array}\right]^{\top}\left[\begin{array}{l}
x_{1} \\
x_{2} \\
x_{3}
\end{array}\right] \mathrm{d} s=\int_{0}^{T}\left(x_{1}-x_{2} Y_{s}-x_{3} X_{s}\right)^{2} \mathrm{~d} s \geqslant 0,
$$

and $\boldsymbol{x}^{\top} \boldsymbol{G}_{T}^{(2)} \boldsymbol{x}=0$ if and only if $x_{1}-x_{2} Y_{s}-x_{3} X_{s}=0$ for all $s \in[0, T]$, which happens with probability 0 , since $\boldsymbol{x}=\left(x_{1}, x_{2}, x_{3}\right)^{\top} \neq \mathbf{0}$ and, for each $s \in(0, T]$, the distribution of $\left(Y_{s}, X_{s}\right)$ is absolutely continuous, because, as in the proof of part (b) in the proof of Theorem A.1 in Bolyog and Pap [11, the conditional distribution of $\left(Y_{s}, X_{s}\right)$ given $\left(Y_{0}, X_{0}\right)$ is absolutely continuous.

Next we are going to show $\widehat{\boldsymbol{\theta}}_{T, n} \stackrel{\mathbb{P}}{\longrightarrow} \widehat{\boldsymbol{\theta}}_{T}$ as $n \rightarrow \infty$. The function $g_{n}$ introduced in (3.4) admits an inverse $g_{n}^{-1}: \mathbb{R} \times(-\infty, 1) \times \mathbb{R}^{2} \times(-\infty, 1) \rightarrow \mathbb{R}^{5}$ satisfying

$$
g_{n}^{-1}(c, d, \delta, \varepsilon, \zeta)=(a, b, \alpha, \beta, \gamma)
$$

with

$$
\begin{gathered}
b=-n \log (1-d), \quad a=\frac{c}{\int_{0}^{\frac{1}{n}} \mathrm{e}^{-b w} \mathrm{~d} w}, \quad \gamma=-n \log (1-\zeta), \\
\beta=\frac{\varepsilon}{\int_{0}^{\frac{1}{n}} \mathrm{e}^{(\gamma-b) w-\frac{\gamma}{n}} \mathrm{~d} w}, \quad \alpha=\frac{\delta+a \beta \int_{0}^{\frac{1}{n}} \mathrm{e}^{\gamma w-\frac{\gamma}{n}}\left(\int_{0}^{w} \mathrm{e}^{-b(w-v)} \mathrm{d} v\right) \mathrm{d} w}{\int_{0}^{\frac{1}{n}} \mathrm{e}^{-\gamma w} \mathrm{~d} w} .
\end{gathered}
$$

Convergence (‥6) yields $\left(\widehat{c}_{T, n}, \widehat{d}_{T, n}, \widehat{\delta}_{T, n}, \widehat{\varepsilon}_{T, n}, \widehat{\zeta}_{T, n}\right) \stackrel{\mathbb{P}}{\longrightarrow} \mathbf{0} \quad$ as $n \rightarrow \quad \infty$, hence $\widehat{d}_{T, n} \in$ $(-\infty, 1)$ and $\widehat{\zeta}_{T, n} \in(-\infty, 1)$ with probability tending to one as $n \rightarrow \infty$. Consequently, $g_{n}^{-1}\left(\widehat{c}_{T, n}, \widehat{d}_{T, n}, \widehat{\delta}_{T, n}, \widehat{\varepsilon}_{T, n}, \widehat{\zeta}_{T, n}\right)=\widehat{\boldsymbol{\theta}}_{T, n}$ with probability tending to one as $n \rightarrow \infty$. We have

$$
\widehat{b}_{T, n}=-n \log \left(1-\widehat{d}_{T, n}\right)=n \widehat{d}_{T, n} h_{1}\left(\widehat{d}_{T, n}\right)
$$

with probability tending to one as $n \rightarrow \infty$, where the continuous function $h_{1}:(-\infty, 1) \rightarrow \mathbb{R}$ is given by

$$
h_{1}(x):= \begin{cases}-\frac{1}{x} \log (1-x) & \text { if } x \neq 0 \\ 1 & \text { if } x=0\end{cases}
$$

By (3.6), we have $n \widehat{d}_{T, n} \stackrel{\mathbb{P}}{\longrightarrow} \widehat{b}_{T}$ and $\widehat{d}_{T, n} \stackrel{\mathbb{P}}{\longrightarrow} 0$, thus we obtain $h_{1}\left(\widehat{d}_{T, n}\right) \stackrel{\mathbb{P}}{\longrightarrow} h_{1}(0)=1$, and hence $\widehat{b}_{T, n} \stackrel{\mathbb{P}}{\longrightarrow} \widehat{b}_{T}$ as $n \rightarrow \infty$.

Moreover,

$$
\widehat{a}_{T, n}=\frac{\widehat{c}_{T, n}}{\int_{0}^{\frac{1}{n}} \mathrm{e}^{-\widehat{b}_{T, n} w} \mathrm{~d} w}=\frac{n \widehat{c}_{T, n}}{n \int_{0}^{\frac{1}{n}} \mathrm{e}^{-\widehat{b}_{T, n} w} \mathrm{~d} w}=\frac{n \widehat{c}_{T, n}}{\int_{0}^{1} \exp \left\{-n^{-1} \widehat{b}_{T, n} v\right\} \mathrm{d} v}=\frac{n \widehat{c}_{T, n}}{h_{2}\left(n^{-1} \widehat{b}_{T, n}\right)}
$$

with probability tending to one as $n \rightarrow \infty$, where the continuous function $h_{2}: \mathbb{R} \rightarrow \mathbb{R}$ is given by

$$
h_{2}(x):=\int_{0}^{1} \mathrm{e}^{-x v} \mathrm{~d} v= \begin{cases}\frac{1-\mathrm{e}^{-x}}{x} & \text { if } x \neq 0 \\ 1 & \text { if } x=0 .\end{cases}
$$


We have already showed $\widehat{b}_{T, n} \stackrel{\mathbb{P}}{\longrightarrow} \widehat{b}_{T}$, yielding $n^{-1} \widehat{b}_{T, n} \stackrel{\mathbb{P}}{\longrightarrow} 0$, and hence $h_{2}\left(n^{-1} \widehat{b}_{T, n}\right) \stackrel{\mathbb{P}}{\longrightarrow} h_{2}(0)=1$ as $n \rightarrow \infty$. By (3.6), we have $n \widehat{c}_{T, n} \stackrel{\mathbb{P}}{\longrightarrow} \widehat{a}_{T}$, thus we obtain $\widehat{a}_{T, n} \stackrel{\mathbb{P}}{\longrightarrow} \widehat{a}_{T}$ as $n \rightarrow \infty$.

In a similar way,

$$
\widehat{\gamma}_{T, n}=-n \log \left(1-\widehat{\zeta}_{T, n}\right)=n \widehat{\zeta}_{T, n} h_{1}\left(\widehat{\zeta}_{T, n}\right)
$$

with probability tending to one as $n \rightarrow \infty$. By (3.6), we have $n \widehat{\zeta}_{T, n} \stackrel{\mathbb{P}}{\longrightarrow} \widehat{\gamma}_{T}$ and $\widehat{\zeta}_{T, n} \stackrel{\mathbb{P}}{\longrightarrow} 0$, thus we obtain $h_{1}\left(\widehat{\zeta}_{T, n}\right) \stackrel{\mathbb{P}}{\longrightarrow} h_{1}(0)=1$, and hence $\widehat{\gamma}_{T, n} \stackrel{\mathbb{P}}{\longrightarrow} \widehat{\gamma}_{T}$ as $n \rightarrow \infty$.

Further,

$$
\widehat{\beta}_{T, n}=\frac{\widehat{\varepsilon}_{T, n}}{\int_{0}^{\frac{1}{n}} \mathrm{e}^{\left(\widehat{\gamma}_{T, n}-\widehat{b}_{T, n}\right) w-\frac{\hat{\gamma}_{T, n}}{n}} \mathrm{~d} w}=\frac{n \widehat{\varepsilon}_{T, n} \mathrm{e}^{\frac{\widehat{\gamma}_{T, n}}{n}}}{h_{2}\left(n^{-1}\left(\widehat{b}_{T, n}-\widehat{\gamma}_{T, n}\right)\right)}
$$

with probability tending to one as $n \rightarrow \infty$. We have already showed $\widehat{b}_{T, n} \stackrel{\mathbb{P}}{\longrightarrow} \widehat{b}_{T}$ and $\widehat{\gamma}_{T, n} \stackrel{\mathbb{P}}{\longrightarrow} \widehat{\gamma}_{T}$, yielding $n^{-1} \widehat{b}_{T, n} \stackrel{\mathbb{P}}{\longrightarrow} 0$ and $n^{-1} \widehat{\gamma}_{T, n} \stackrel{\mathbb{P}}{\longrightarrow} 0$, and hence $\mathrm{e}^{\frac{\hat{\gamma}_{T, n}}{n}} \stackrel{\mathbb{P}}{\longrightarrow} 1$ and $h_{2}\left(n^{-1}\left(\widehat{b}_{T, n}-\widehat{\gamma}_{T, n}\right)\right) \stackrel{\mathbb{P}}{\longrightarrow}$

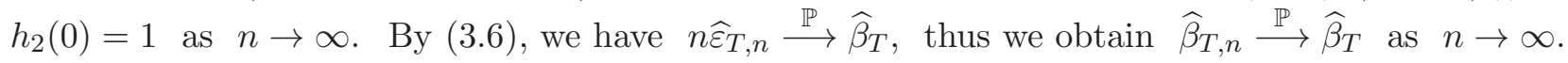

Finally,

$$
\widehat{\alpha}_{T, n}=\frac{\widehat{\delta}_{T, n}+\widehat{a}_{T, n} \widehat{\beta}_{T, n} \int_{0}^{\frac{1}{n}} \mathrm{e}^{\widehat{\gamma}_{T, n} w-\frac{\widehat{\gamma}_{T, n}}{n}}\left(\int_{0}^{w} \mathrm{e}^{-b(w-v)} \mathrm{d} v\right) \mathrm{d} w}{\int_{0}^{\frac{1}{n}} \mathrm{e}^{-\widehat{\gamma}_{T, n} w} \mathrm{~d} w}=\frac{n \widehat{\delta}_{T, n}+\widehat{a}_{T, n} \widehat{\beta}_{T, n} \mathrm{e}^{-\frac{\widehat{\gamma}_{T, n}}{n}} I_{T, n}}{h_{2}\left(n^{-1} \widehat{\gamma}_{T, n}\right)}
$$

with probability tending to one as $n \rightarrow \infty$, where

$$
I_{T, n}=n \int_{0}^{\frac{1}{n}} \mathrm{e}^{\widehat{\gamma}_{T, n} w}\left(\int_{0}^{w} \mathrm{e}^{-b(w-v)} \mathrm{d} v\right) \mathrm{d} w \leqslant \frac{1}{n} \mathrm{e}^{\frac{\left|\hat{\gamma}_{T, n}\right|}{n}} \mathrm{e}^{\frac{\left|\widehat{b}_{T, n}\right|}{n}}
$$

We have already showed $\widehat{a}_{T, n} \stackrel{\mathbb{P}}{\longrightarrow} \widehat{a}_{T}, \widehat{b}_{T, n} \stackrel{\mathbb{P}}{\longrightarrow} \widehat{b}_{T}, \quad \widehat{\beta}_{T, n} \stackrel{\mathbb{P}}{\longrightarrow} \widehat{\beta}_{T}$ and $\widehat{\gamma}_{T, n} \stackrel{\mathbb{P}}{\longrightarrow} \widehat{\gamma}_{T}$, yielding $n^{-1} \widehat{b}_{T, n} \stackrel{\mathbb{P}}{\longrightarrow} 0$ and $n^{-1} \widehat{\gamma}_{T, n} \stackrel{\mathbb{P}}{\longrightarrow} 0$, and hence $h_{2}\left(n^{-1} \widehat{\gamma}_{T, n}\right) \stackrel{\mathbb{P}}{\longrightarrow} h_{2}(0)=1$, e $\stackrel{\widehat{\gamma}_{T, n}}{n} \stackrel{\mathbb{P}}{\longrightarrow} 1$, e $\stackrel{\left|\hat{\gamma}_{T, n}\right|}{n} \stackrel{\mathbb{P}}{\longrightarrow} 1$ and $\mathrm{e}^{\frac{\left|\hat{b}_{T, n}\right|}{n}} \stackrel{\mathbb{P}}{\longrightarrow} 1$, implying $I_{T, n} \stackrel{\mathbb{P}}{\longrightarrow} 0$ as $n \rightarrow \infty$. By (3.6), we have $n \widehat{\delta}_{T, n} \stackrel{\mathbb{P}}{\longrightarrow} \widehat{\alpha}_{T}$, thus we obtain $\widehat{\alpha}_{T, n} \stackrel{\mathbb{P}}{\longrightarrow} \widehat{\alpha}_{T}$ as $n \rightarrow \infty$.

Using the SDE (1.1) and Corollary 3.2.20 in Karatzas and Shreve 24], one can check that

$$
\widehat{\boldsymbol{\theta}}_{T}-\boldsymbol{\theta}=\left[\begin{array}{c}
\widehat{a}_{T}-a \\
\widehat{b}_{T}-b \\
\widehat{\alpha}_{T}-\alpha \\
\widehat{\beta}_{T}-\beta \\
\widehat{\gamma}_{T}-\gamma
\end{array}\right]=\left[\begin{array}{l}
\left(\boldsymbol{G}_{T}^{(1)}\right)^{-1} \boldsymbol{h}_{T}^{(1)} \\
\left(\boldsymbol{G}_{T}^{(2)}\right)^{-1} \boldsymbol{h}_{T}^{(2)}
\end{array}\right]=\boldsymbol{G}_{T}^{-1} \boldsymbol{h}_{T}
$$

on the event where the random matrices $\boldsymbol{G}_{T}^{(1)}$ and $\boldsymbol{G}_{T}^{(2)}$ are invertible, where

$$
\boldsymbol{G}_{T}:=\left[\begin{array}{cc}
\boldsymbol{G}_{T}^{(1)} & \mathbf{0} \\
\mathbf{0} & \boldsymbol{G}_{T}^{(2)}
\end{array}\right], \quad \boldsymbol{h}_{T}:=\left[\begin{array}{l}
\boldsymbol{h}_{T}^{(1)} \\
\boldsymbol{h}_{T}^{(2)}
\end{array}\right]
$$

with

$$
\boldsymbol{h}_{T}^{(1)}:=\sigma_{1} \int_{0}^{T} \sqrt{Y_{s}}\left[\begin{array}{c}
1 \\
-Y_{s}
\end{array}\right] \mathrm{d} W_{s}, \quad \boldsymbol{h}_{T}^{(2)}:=\int_{0}^{T}\left[\begin{array}{c}
1 \\
-Y_{s} \\
-X_{s}
\end{array}\right]\left(\sigma_{2} \sqrt{Y_{s}} \mathrm{~d} \widetilde{W}_{s}+\sigma_{3} \mathrm{~d} L_{s}\right)
$$


where

$$
\widetilde{W}_{s}:=\varrho W_{s}+\sqrt{1-\varrho^{2}} B_{s}, \quad s \in \mathbb{R}_{+},
$$

is a standard Wiener process, independent of $L$. Indeed,

$$
\widehat{\boldsymbol{\theta}}_{T}=\boldsymbol{G}_{T}^{-1} \boldsymbol{f}_{T} \quad \text { with } \quad \boldsymbol{f}_{T}:=\left[\begin{array}{l}
\boldsymbol{f}_{T}^{(1)} \\
\boldsymbol{f}_{T}^{(2)}
\end{array}\right],
$$

hence $\widehat{\boldsymbol{\theta}}_{T}-\boldsymbol{\theta}=\boldsymbol{G}_{T}^{-1}\left(\boldsymbol{f}_{T}-\boldsymbol{G}_{T} \boldsymbol{\theta}\right)$, where

$$
\boldsymbol{f}_{T}-\boldsymbol{G}_{T} \boldsymbol{\theta}=\left[\begin{array}{cc}
\boldsymbol{f}_{T}^{(1)}-\boldsymbol{G}_{T}^{(1)} & {\left[\begin{array}{l}
a \\
b
\end{array}\right]} \\
\boldsymbol{f}_{T}^{(2)}-\boldsymbol{G}_{T}^{(2)}\left[\begin{array}{l}
\alpha \\
\beta \\
\gamma
\end{array}\right]
\end{array}\right] .
$$

Using the first equation of SDE (1.1) and Corollary 3.2.20 in Karatzas and Shreve [24], we obtain

$$
\begin{aligned}
\boldsymbol{f}_{T}^{(1)}-\boldsymbol{G}_{T}^{(1)}\left[\begin{array}{l}
a \\
b
\end{array}\right] & =\int_{0}^{T}\left[\begin{array}{c}
1 \\
-Y_{s}
\end{array}\right] \mathrm{d} Y_{s}-\int_{0}^{T}\left[\begin{array}{c}
1 \\
-Y_{s}
\end{array}\right]\left[\begin{array}{c}
1 \\
-Y_{s}
\end{array}\right]^{\top} \mathrm{d} s\left[\begin{array}{l}
a \\
b
\end{array}\right] \\
& =\int_{0}^{T}\left[\begin{array}{c}
1 \\
-Y_{s}
\end{array}\right]\left(\mathrm{d} Y_{s}-\left[\begin{array}{c}
1 \\
-Y_{s}
\end{array}\right]^{\top}\left[\begin{array}{l}
a \\
b
\end{array}\right] \mathrm{d} s\right)=\int_{0}^{T}\left[\begin{array}{c}
1 \\
-Y_{s}
\end{array}\right]\left(\mathrm{d} Y_{s}-\left(a-b Y_{s}\right) \mathrm{d} s\right) \\
& =\int_{0}^{T}\left[\begin{array}{c}
1 \\
-Y_{s}
\end{array}\right] \sigma_{1} \sqrt{Y_{s}} \mathrm{~d} W_{s} .
\end{aligned}
$$

In a similar way,

$$
\begin{aligned}
& \boldsymbol{f}_{T}^{(2)}-\boldsymbol{G}_{T}^{(2)}\left[\begin{array}{l}
\alpha \\
\beta \\
\gamma
\end{array}\right]=\int_{0}^{T}\left[\begin{array}{c}
1 \\
-Y_{s} \\
-X_{s}
\end{array}\right] \mathrm{d} X_{s}-\int_{0}^{T}\left[\begin{array}{c}
1 \\
-Y_{s} \\
-X_{s}
\end{array}\right]\left[\begin{array}{c}
1 \\
-Y_{s} \\
-X_{s}
\end{array}\right]^{\top} \mathrm{d} s\left[\begin{array}{l}
\alpha \\
\beta \\
\gamma
\end{array}\right] \\
& =\int_{0}^{T}\left[\begin{array}{c}
1 \\
-Y_{s} \\
-X_{s}
\end{array}\right]\left(\mathrm{d} X_{s}-\left[\begin{array}{c}
1 \\
-Y_{s} \\
-X_{s}
\end{array}\right]^{\top}\left[\begin{array}{l}
\alpha \\
\beta \\
\gamma
\end{array}\right] \mathrm{d} s\right)=\int_{0}^{T}\left[\begin{array}{c}
1 \\
-Y_{s} \\
-X_{s}
\end{array}\right]\left(\mathrm{d} X_{s}-\left(\alpha-\beta Y_{s}-\gamma X_{s}\right) \mathrm{d} s\right) \\
& =\int_{0}^{T}\left[\begin{array}{c}
1 \\
-Y_{s} \\
-X_{s}
\end{array}\right]\left(\sigma_{2} \sqrt{Y_{s}}\left[\varrho \mathrm{d} W_{s}+\sqrt{1-\varrho^{2}} B_{s}\right]+\sigma_{3} \mathrm{~d} L_{s}\right) .
\end{aligned}
$$

\section{Consistency of CLSE}

First we consider the case of subcritical Heston models, i.e., when $b \in \mathbb{R}_{++}$. 
4.1 Theorem. Let us consider the two-factor affine diffusion model (1.1) with $a, b \in \mathbb{R}_{++}, \alpha, \beta \in \mathbb{R}$, $\gamma \in \mathbb{R}_{++}, \sigma_{1} \in \mathbb{R}_{++}, \sigma_{2}, \sigma_{3} \in \mathbb{R}_{+}$and $\varrho \in[-1,1]$ with a random initial value $\left(\eta_{0}, \zeta_{0}\right)$ independent of $\left(W_{t}, B_{t}, L_{t}\right)_{t \in \mathbb{R}_{+}}$satisfying $\mathbb{P}\left(\eta_{0} \in \mathbb{R}_{+}\right)=1$. Suppose that $\left(1-\varrho^{2}\right) \sigma_{2}^{2}+\sigma_{3}^{2}>0$. Then the CLSE of $\boldsymbol{\theta}=(a, b, \alpha, \beta, \gamma)^{\top}$ is strongly consistent, i.e., $\widehat{\boldsymbol{\theta}}_{T}=\left(\widehat{a}_{T}, \widehat{b}_{T}, \widehat{\alpha}_{T}, \widehat{\beta}_{T}, \widehat{\gamma}_{T}\right)^{\top} \stackrel{\text { a.s. }}{\longrightarrow \boldsymbol{\theta}}=(a, b, \alpha, \beta, \gamma)^{\top}$ as $T \rightarrow \infty$.

Proof. By (3.7), we have

$$
\widehat{\boldsymbol{\theta}}_{T}-\boldsymbol{\theta}=\left(T^{-1} \boldsymbol{G}_{T}\right)^{-1}\left(T^{-1} \boldsymbol{h}_{T}\right)
$$

on the event, where the random matrix $\boldsymbol{G}_{T}$ is invertible, which has propapility 1, see Lemma 3.3 .

By Theorem A.2, we obtain

$$
T^{-1} \boldsymbol{G}_{T} \stackrel{\text { a.s. }}{\longrightarrow} \mathbb{E}\left(\boldsymbol{G}_{\infty}\right) \quad \text { as } T \rightarrow \infty
$$

where

$$
\boldsymbol{G}_{\infty}:=\left[\begin{array}{cc}
\boldsymbol{G}_{\infty}^{(1)} & \mathbf{0} \\
\mathbf{0} & \boldsymbol{G}_{\infty}^{(2)}
\end{array}\right]
$$

with

$$
\boldsymbol{G}_{\infty}^{(1)}:=\left[\begin{array}{cc}
1 & -Y_{\infty} \\
-Y_{\infty} & Y_{\infty}^{2}
\end{array}\right], \quad \boldsymbol{G}_{\infty}^{(2)}:=\left[\begin{array}{ccc}
1 & -Y_{\infty} & -X_{\infty} \\
-Y_{\infty} & Y_{\infty}^{2} & Y_{\infty} X_{\infty} \\
-X_{\infty} & Y_{\infty} X_{\infty} & X_{\infty}^{2}
\end{array}\right],
$$

where the random vector $\left(Y_{\infty}, X_{\infty}\right)$ is given by Theorem A.1, since, by Theorem B.2, the entries of $\mathbb{E}\left(\boldsymbol{G}_{\infty}\right)$ exist and finite.

The matrix $\mathbb{E}\left(\boldsymbol{G}_{\infty}^{(1)}\right)$ is strictly positive definite, since for all $\boldsymbol{x} \in \mathbb{R}^{2} \backslash\{\mathbf{0}\}$, we have $\boldsymbol{x}^{\top} \mathbb{E}\left(\boldsymbol{G}_{\infty}^{(1)}\right) \boldsymbol{x}>$ 0. Indeed, for all $\boldsymbol{x}=\left(x_{1}, x_{2}\right)^{\top} \in \mathbb{R}^{2} \backslash\{\mathbf{0}\}$,

$$
\left[\begin{array}{l}
x_{1} \\
x_{2}
\end{array}\right]^{\top} \mathbb{E}\left(\boldsymbol{G}_{\infty}^{(1)}\right)\left[\begin{array}{l}
x_{1} \\
x_{2}
\end{array}\right]=\mathbb{E}\left[\left(x_{1}-x_{2} Y_{\infty}\right)^{2}\right]>0,
$$

since, by Theorem A.2, the distribution of $Y_{\infty}$ is absolutely continuous, hence $x_{1}-x_{2} Y_{\infty} \neq 0$ with probability 1 . In a similar way, the matrix $\mathbb{E}\left(\boldsymbol{G}_{\infty}^{(2)}\right)$ is strictly positive definite, since for all $\boldsymbol{x} \in \mathbb{R}^{3} \backslash\{\mathbf{0}\}$, we have $\boldsymbol{x}^{\top} \mathbb{E}\left(\boldsymbol{G}_{\infty}^{(2)}\right) \boldsymbol{x}>0$. Indeed, for all $\boldsymbol{x}=\left(x_{1}, x_{2}, x_{3}\right)^{\top} \in \mathbb{R}^{3} \backslash\{\mathbf{0}\}$,

$$
\left[\begin{array}{l}
x_{1} \\
x_{2} \\
x_{3}
\end{array}\right]^{\top} \mathbb{E}\left(\boldsymbol{G}_{\infty}^{(2)}\right)\left[\begin{array}{l}
x_{1} \\
x_{2} \\
x_{3}
\end{array}\right]=\mathbb{E}\left[\left(x_{1}-x_{2} Y_{\infty}-x_{3} Z_{\infty}\right)^{2}\right]>0
$$

since, by Theorem A.2, the distribution of $\left(Y_{\infty}, X_{\infty}\right)$ is absolutely continuous, hence $x_{1}-x_{2} Y_{\infty}-$ $x_{3} X_{\infty} \neq 0$ with probability 1 . Thus the matrices $\mathbb{E}\left(\boldsymbol{G}_{\infty}^{(1)}\right)$ and $\mathbb{E}\left(\boldsymbol{G}_{\infty}^{(2)}\right)$ are invertible, whence we conclude

$$
\left(T^{-1} \boldsymbol{G}_{T}\right)^{-1} \stackrel{\text { a.s. }}{\longrightarrow}\left[\begin{array}{cc}
{\left[\mathbb{E}\left(\boldsymbol{G}_{\infty}^{(1)}\right)\right]^{-1}} & \mathbf{0} \\
\mathbf{0} & {\left[\mathbb{E}\left(\boldsymbol{G}_{\infty}^{(2)}\right)\right]^{-1}}
\end{array}\right]=\left[\mathbb{E}\left(\boldsymbol{G}_{\infty}\right)\right]^{-1} \quad \text { as } T \rightarrow \infty .
$$


The aim of the next discussion is to show convergence

$$
T^{-1} \boldsymbol{h}_{T} \stackrel{\text { a.s. }}{\longrightarrow} \mathbf{0} \quad \text { as } T \rightarrow \infty .
$$

We have

$$
\frac{1}{T} \int_{0}^{T} \sqrt{Y_{s}} \mathrm{~d} W_{s}=\frac{1}{T} \int_{0}^{T} Y_{s} \mathrm{~d} s \cdot \frac{\int_{0}^{T} \sqrt{Y_{s}} \mathrm{~d} W_{s}}{\int_{0}^{T} Y_{s} \mathrm{~d} s} \stackrel{\text { a.s. }}{\longrightarrow} 0 \quad \text { as } T \rightarrow \infty .
$$

Indeed, we have already proved

$$
\frac{1}{T} \int_{0}^{T} Y_{s} \mathrm{~d} s \stackrel{\text { a.s. }}{\longrightarrow} \mathbb{E}\left(Y_{\infty}\right)=\frac{a}{b} \in \mathbb{R}_{++} \quad \text { as } T \rightarrow \infty,
$$

and the strong law of large numbers for continuous local martingales (see, e.g., Theorem D.1) implies

$$
\frac{\int_{0}^{T} \sqrt{Y_{s}} \mathrm{~d} W_{s}}{\int_{0}^{T} Y_{s} \mathrm{~d} s} \stackrel{\text { a.s. }}{\longrightarrow} 0 \quad \text { as } T \rightarrow \infty
$$

since we have

$$
\int_{0}^{T} Y_{s} \mathrm{~d} s=T \cdot \frac{1}{T} \int_{0}^{T} Y_{s} \mathrm{~d} s \stackrel{\text { a.s. }}{\rightarrow} \infty \quad \text { as } T \rightarrow \infty .
$$

Further,

$$
\frac{1}{T} \int_{0}^{T}\left(\sigma_{2} \sqrt{Y_{s}} \mathrm{~d} \widetilde{W}_{s}+\sigma_{3} \mathrm{~d} L_{s}\right)=\frac{1}{T} \int_{0}^{T}\left(\sigma_{2}^{2} Y_{s}+\sigma_{3}^{2}\right) \mathrm{d} s \cdot \frac{\int_{0}^{T}\left(\sigma_{2} \sqrt{Y_{s}} \mathrm{~d} \widetilde{W}_{s}+\sigma_{3} \mathrm{~d} L_{s}\right)}{\int_{0}^{T}\left(\sigma_{2}^{2} Y_{s}+\sigma_{3}^{2}\right) \mathrm{d} s} \stackrel{\text { a.s. }}{\longrightarrow} 0
$$

as $T \rightarrow \infty$. Indeed, we have already proved

$$
\frac{1}{T} \int_{0}^{T}\left(\sigma_{2}^{2} Y_{s}+\sigma_{3}^{2}\right) \mathrm{d} s \stackrel{\text { a.s. }}{\longrightarrow} \mathbb{E}\left(\sigma_{2}^{2} Y_{\infty}+\sigma_{3}^{2}\right)=\sigma_{2}^{2} \frac{a}{b}+\sigma_{3}^{2} \in \mathbb{R}_{++} \quad \text { as } \quad T \rightarrow \infty,
$$

and the strong law of large numbers for continuous local martingales (see, e.g., Theorem D.1) implies

$$
\frac{\int_{0}^{T}\left(\sigma_{2} \sqrt{Y_{s}} \mathrm{~d} \widetilde{W}_{s}+\sigma_{3} \mathrm{~d} L_{s}\right)}{\int_{0}^{T}\left(\sigma_{2}^{2} Y_{s}+\sigma_{3}^{2}\right) \mathrm{d} s} \stackrel{\text { a.s. }}{\longrightarrow} 0 \quad \text { as } T \rightarrow \infty
$$

since we have

$$
\int_{0}^{T}\left(\sigma_{2}^{2} Y_{s}+\sigma_{3}^{2}\right) \mathrm{d} s=T \cdot \frac{1}{T} \int_{0}^{T}\left(\sigma_{2}^{2} Y_{s}+\sigma_{3}^{2}\right) \mathrm{d} s \stackrel{\text { a.s. }}{\longrightarrow} \infty \quad \text { as } \quad T \rightarrow \infty .
$$

One can check

$$
\begin{gathered}
\frac{1}{T} \int_{0}^{T} Y_{s} \sqrt{Y_{s}} \mathrm{~d} W_{s} \stackrel{\text { a.s. }}{\longrightarrow} 0 \\
\frac{1}{T} \int_{0}^{T} Y_{s}\left(\sigma_{2} \sqrt{Y_{s}} \mathrm{~d} \widetilde{W}_{s}+\sigma_{3} \mathrm{~d} L_{s}\right) \stackrel{\text { a.s. }}{\longrightarrow} 0, \quad \frac{1}{T} \int_{0}^{T} X_{s}\left(\sigma_{2} \sqrt{Y_{s}} \mathrm{~d} \widetilde{W}_{s}+\sigma_{3} \mathrm{~d} L_{s}\right) \stackrel{\text { a.s. }}{\longrightarrow} 0
\end{gathered}
$$


as $T \rightarrow \infty$ in the same way, since

$$
\begin{gathered}
\frac{1}{T} \int_{0}^{T} Y_{s}^{3} \mathrm{~d} s \stackrel{\text { a.s. }}{\longrightarrow} \mathbb{E}\left(Y_{\infty}^{3}\right) \in \mathbb{R}_{++}, \\
\frac{1}{T} \int_{0}^{T} Y_{s}^{2}\left(\sigma_{2}^{2} Y_{s}+\sigma_{3}^{2}\right) \mathrm{d} s \stackrel{\text { a.s. }}{\longrightarrow} \mathbb{E}\left[Y_{s}^{2}\left(\sigma_{2}^{2} Y_{s}+\sigma_{3}^{2}\right)\right] \in \mathbb{R}_{++}, \\
\frac{1}{T} \int_{0}^{T} X_{s}^{2}\left(\sigma_{2}^{2} Y_{s}+\sigma_{3}^{2}\right) \mathrm{d} s \stackrel{\text { a.s. }}{\longrightarrow} \mathbb{E}\left[X_{s}^{2}\left(\sigma_{2}^{2} Y_{s}+\sigma_{3}^{2}\right)\right] \in \mathbb{R}_{++}
\end{gathered}
$$

as $T \rightarrow \infty$. Consequently, we conclude (4.5). Finally, by (4.4) and (4.5), we obtain the statement.

In order to handle supercritical two-factor affine diffusion models when $b \in \mathbb{R}_{--}$, we need the following integral version of the Toeplitz Lemma, due to Dietz and Kutoyants [14].

4.2 Lemma. Let $\left\{\varphi_{T}: T \in \mathbb{R}_{+}\right\}$be a family of probability measures on $\mathbb{R}_{+}$such that $\varphi_{T}([0, T])=1$ for all $T \in \mathbb{R}_{+}$, and $\lim _{T \rightarrow \infty} \varphi_{T}([0, K])=0$ for all $K \in \mathbb{R}_{++}$. Then for every bounded and measurable function $f: \mathbb{R}_{+} \rightarrow \mathbb{R}$ for which the limit $f(\infty):=\lim _{t \rightarrow \infty} f(t)$ exists, we have

$$
\lim _{T \rightarrow \infty} \int_{0}^{\infty} f(t) \varphi_{T}(\mathrm{~d} t)=f(\infty)
$$

As a special case, we have the following integral version of the Kronecker Lemma, see Küchler and Sørensen [25, Lemma B.3.2].

4.3 Lemma. Let $a: \mathbb{R}_{+} \rightarrow \mathbb{R}_{+}$be a measurable function. Put $b(T):=\int_{0}^{T} a(t) \mathrm{d} t, \quad T \in \mathbb{R}_{+}$. Suppose that $\lim _{T \rightarrow \infty} b(T)=\infty$. Then for every bounded and measurable function $f: \mathbb{R}_{+} \rightarrow \mathbb{R}$ for which the limit $f(\infty):=\lim _{t \rightarrow \infty} f(t)$ exists, we have

$$
\lim _{T \rightarrow \infty} \frac{1}{b(T)} \int_{0}^{T} a(t) f(t) \mathrm{d} t=f(\infty) .
$$

Next we present an auxiliary lemma in the supercritical case about the asymptotic behavior of $Y_{t}$ as $t \rightarrow \infty$.

4.4 Lemma. Let us consider the two-factor affine diffusion model (1.1) with $a \in \mathbb{R}_{+}, \quad b \in \mathbb{R}_{--}$, $\alpha, \beta, \gamma \in \mathbb{R}, \quad \sigma_{1}, \sigma_{2}, \sigma_{3} \in \mathbb{R}_{+}$and $\varrho \in[-1,1]$ with a random initial value $\left(\eta_{0}, \zeta_{0}\right)$ independent of $\left(W_{t}, B_{t}, L_{t}\right)_{t \in \mathbb{R}_{+}}$satisfying $\mathbb{P}\left(\eta_{0} \in \mathbb{R}_{+}\right)=1$. Then there exists a random variable $V_{Y}$ such that

$$
\mathrm{e}^{b t} Y_{t} \stackrel{\text { a.s. }}{\rightarrow} V_{Y} \quad \text { as } t \rightarrow \infty
$$

with $\mathbb{P}\left(V_{Y} \neq 0\right)=1$, and, for each $k \in \mathbb{N}$,

$$
\mathrm{e}^{k b t} \int_{0}^{t} Y_{u}^{k} \mathrm{~d} u \stackrel{\text { a.s. }}{\longrightarrow}-\frac{V_{Y}^{k}}{k b} \quad \text { as } t \rightarrow \infty .
$$

Proof. By (2.1),

$$
\mathbb{E}\left(Y_{t} \mid \mathcal{F}_{s}\right)=\mathbb{E}\left(Y_{t} \mid Y_{s}\right)=\mathrm{e}^{-b(t-s)} Y_{s}+a \int_{s}^{t} \mathrm{e}^{-b(t-u)} \mathrm{d} u
$$


for all $s, t \in \mathbb{R}_{+}$with $0 \leqslant s \leqslant t$. Thus

$$
\mathbb{E}\left(\mathrm{e}^{b t} Y_{t} \mid \mathcal{F}_{s}^{Y}\right)=\mathrm{e}^{b s} Y_{s}+a \int_{s}^{t} \mathrm{e}^{b u} \mathrm{~d} u \geqslant \mathrm{e}^{b s} Y_{s}
$$

for all $s, t \in \mathbb{R}_{+}$with $0 \leqslant s \leqslant t$, consequently, the process $\left(\mathrm{e}^{b t} Y_{t}\right)_{t \in \mathbb{R}_{+}}$is a non-negative submartingale with respect to the filtration $\left(\mathcal{F}_{t}^{Y}\right)_{t \in \mathbb{R}_{+}}$. Moreover, $b \in \mathbb{R}_{--}$implies

$$
\mathbb{E}\left(\mathrm{e}^{b t} Y_{t}\right)=y_{0}+a \int_{0}^{t} \mathrm{e}^{b u} \mathrm{~d} u \leqslant y_{0}+a \int_{0}^{\infty} \mathrm{e}^{b u} \mathrm{~d} u=y_{0}-\frac{a}{b}<\infty, \quad t \in \mathbb{R}_{+},
$$

hence, by the submartingale convergence theorem, there exists a non-negative random variable $V_{Y}$ such that (4.6) holds.

The distribution of $V_{Y}$ coincides with the distribution of $\widetilde{\mathcal{Y}}_{-1 / b}$, where $\left(\widetilde{\mathcal{Y}}_{t}\right)_{t \in \mathbb{R}_{+}}$is a CIR process given by the SDE

$$
\mathrm{d} \widetilde{\mathcal{Y}}_{t}=a \mathrm{~d} t+\sigma_{1} \sqrt{\widetilde{\mathcal{Y}}_{t}} \mathrm{~d} \mathcal{W}_{t}, \quad t \in \mathbb{R}_{+},
$$

with initial value $\widetilde{\mathcal{Y}}_{0}=y_{0}$, where $\left(\mathcal{W}_{t}\right)_{t \in \mathbb{R}_{+}}$is a standard Wiener process, see Ben Alaya and Kebaier [9, Proposition 3]. Consequently, $\mathbb{P}\left(V_{Y} \in \mathbb{R}_{++}\right)=1$, since $\widetilde{\mathcal{Y}}_{t}, t \in \mathbb{R}_{++}$, are absolutely continuous random variables.

If $\omega \in \Omega$ such that $\mathbb{R}_{+} \ni t \mapsto Y_{t}(\omega)$ is continuous and $\mathrm{e}^{b t} Y_{t}(\omega) \rightarrow V_{Y}(\omega)$ as $t \rightarrow \infty$, then, by the integral Kronecker Lemma 4.3 with $f(t)=\mathrm{e}^{k b t} Y_{t}(\omega)^{k}$ and $a(t)=\mathrm{e}^{-k b t}, t \in \mathbb{R}_{+}$, we have

$$
\frac{1}{\int_{0}^{t} \mathrm{e}^{-k b u} \mathrm{~d} u} \int_{0}^{t} \mathrm{e}^{-k b u}\left(\mathrm{e}^{k b u} Y_{u}(\omega)^{k}\right) \mathrm{d} u \rightarrow V_{Y}(\omega)^{k} \quad \text { as } t \rightarrow \infty .
$$

Here $\int_{0}^{t} \mathrm{e}^{-k b u} \mathrm{~d} u=-\frac{\mathrm{e}^{-k b t}-1}{k b}, t \in \mathbb{R}_{+}$, thus we conclude the second convergence in (4.7).

The next theorem states strong consistency of the CLSE of $b$ in the supercritical case.

4.5 Theorem. Let us consider the two-factor affine diffusion model (1.1) with $a \in \mathbb{R}_{+}, \quad b \in \mathbb{R}_{--}$, $\alpha, \beta, \gamma \in \mathbb{R}, \sigma_{1} \in \mathbb{R}_{++}, \sigma_{2}, \sigma_{3} \in \mathbb{R}_{+}$and $\varrho \in[-1,1]$ with a random initial value $\left(\eta_{0}, \zeta_{0}\right)$ independent of $\left(W_{t}, B_{t}, L_{t}\right)_{t \in \mathbb{R}_{+}}$satisfying $\mathbb{P}\left(\eta_{0} \in \mathbb{R}_{+}\right)=1$. Then the CLSE of $b$ is strongly consistent, i.e., $\widehat{b}_{T} \stackrel{\text { a.s. }}{\longrightarrow} b$ as $T \rightarrow \infty$.

Proof. By Lemma 3.3, there exists a unique CLSE $\widehat{b}_{T}$ of $b$ for all $T \in \mathbb{R}_{++}$which has the form given in (3.6). By Ito's formula,

$$
\int_{0}^{T} Y_{s} \mathrm{~d} Y_{s}=\frac{1}{2}\left(Y_{T}^{2}-Y_{0}^{2}\right)-\frac{1}{2} \sigma_{1}^{2} \int_{0}^{T} Y_{s} \mathrm{~d} s, \quad T \in \mathbb{R}_{+},
$$

hence, by (4.6) and (4.7), we have

$$
\begin{aligned}
\widehat{b}_{T} & =\frac{\left(Y_{T}-Y_{0}\right) \int_{0}^{T} Y_{s} \mathrm{~d} s-T \int_{0}^{T} Y_{s} \mathrm{~d} Y_{s}}{T \int_{0}^{T} Y_{s}^{2} \mathrm{~d} s-\left(\int_{0}^{T} Y_{s} \mathrm{~d} s\right)^{2}}=\frac{\left(Y_{T}-Y_{0}\right) \int_{0}^{T} Y_{s} \mathrm{~d} s-\frac{T}{2}\left(Y_{T}^{2}-Y_{0}^{2}\right)+\frac{T}{2} \sigma_{1}^{2} \int_{0}^{T} Y_{s} \mathrm{~d} s}{T \int_{0}^{T} Y_{s}^{2} \mathrm{~d} s-\left(\int_{0}^{T} Y_{s} \mathrm{~d} s\right)^{2}} \\
& =\frac{\frac{1}{T}\left(\mathrm{e}^{b T} Y_{T}-\mathrm{e}^{b T} Y_{0}\right)\left(\mathrm{e}^{b T} \int_{0}^{T} Y_{s} \mathrm{~d} s\right)-\frac{1}{2}\left(\mathrm{e}^{2 b T} Y_{T}^{2}-\mathrm{e}^{2 b T} Y_{0}^{2}\right)+\frac{1}{2} \sigma_{1}^{2} \mathrm{e}^{b T}\left(\mathrm{e}^{b T} \int_{0}^{T} Y_{s} \mathrm{~d} s\right)}{\mathrm{e}^{2 b T} \int_{0}^{T} Y_{s}^{2} \mathrm{~d} s-\frac{1}{T}\left(\mathrm{e}^{b T} \int_{0}^{T} Y_{s} \mathrm{~d} s\right)^{2}} \\
& \stackrel{\text { a.s. }}{\longrightarrow} \frac{0\left(V_{Y}-0\right)\left(-\frac{V_{Y}}{b}\right)-\frac{1}{2}\left(V_{Y}^{2}-0\right)+\frac{1}{2} \sigma_{1}^{2} 0\left(-\frac{V_{Y}}{b}\right)}{-\frac{V_{Y}^{2}}{2 b}-0\left(-\frac{V_{Y}}{b}\right)^{2}}=b
\end{aligned}
$$

as $T \rightarrow \infty$. 
4.6 Remark. For critical two-factor affine diffusion models, it will turn out that the CLSE of $a$ and $\alpha$ are not even weakly consistent, but the CLSE of $b, \beta$ and $\gamma$ are weakly consistent, see Theorem 6.2 .

4.7 Remark. For supercritical two-factor affine diffusion models, it will turn out that the CLSE of $a$ and $\alpha$ are not even weakly consistent, but the CLSE of $\beta$ and $\gamma$ are weakly consistent, see Theorem 7.3 ,

\section{Asymptotic behavior of CLSE: subcritical case}

5.1 Theorem. Let us consider the two-factor affine diffusion model (1.1) with $a, b \in \mathbb{R}_{++}, \alpha, \beta \in \mathbb{R}$, $\gamma \in \mathbb{R}_{++}, \sigma_{1} \in \mathbb{R}_{++}, \sigma_{2}, \sigma_{3} \in \mathbb{R}_{+}$and $\varrho \in[-1,1]$ with a random initial value $\left(\eta_{0}, \zeta_{0}\right)$ independent of $\left(W_{t}, B_{t}, L_{t}\right)_{t \in \mathbb{R}_{+}}$satisfying $\mathbb{P}\left(\eta_{0} \in \mathbb{R}_{+}\right)=1$. Suppose that $\left(1-\varrho^{2}\right) \sigma_{2}^{2}+\sigma_{3}^{2}>0$. Then the CLSE of $\boldsymbol{\theta}=(a, b, \alpha, \beta, \gamma)^{\top}$ is asymptotically normal, namely,

$$
T^{\frac{1}{2}}\left(\widehat{\boldsymbol{\theta}}_{T}-\boldsymbol{\theta}\right) \stackrel{\mathcal{D}}{\longrightarrow} \mathcal{N}_{5}\left(\mathbf{0},\left[\mathbb{E}\left(\boldsymbol{G}_{\infty}\right)\right]^{-1} \mathbb{E}\left(\widetilde{\boldsymbol{G}}_{\infty}\right)\left[\mathbb{E}\left(\boldsymbol{G}_{\infty}\right)\right]^{-1}\right) \quad \text { as } T \rightarrow \infty
$$

where $\boldsymbol{G}_{\infty}$ is given in (4.3) and $\widetilde{\boldsymbol{G}}_{\infty}$ has the form

$$
\left[\begin{array}{ccccc}
\sigma_{1}^{2} Y_{\infty} & -\sigma_{1}^{2} Y_{\infty}^{2} & \varrho \sigma_{1} \sigma_{2} Y_{\infty} & -\varrho \sigma_{1} \sigma_{2} Y_{\infty}^{2} & -\varrho \sigma_{1} \sigma_{2} Y_{\infty} X_{\infty} \\
-\sigma_{1}^{2} Y_{\infty}^{2} & \sigma_{1}^{2} Y_{\infty}^{3} & -\varrho \sigma_{1} \sigma_{2} Y_{\infty}^{2} & \varrho \sigma_{1} \sigma_{2} Y_{\infty}^{3} & \varrho \sigma_{1} \sigma_{2} Y_{\infty}^{2} X_{\infty} \\
\varrho \sigma_{1} \sigma_{2} Y_{\infty} & -\varrho \sigma_{1} \sigma_{2} Y_{\infty}^{2} & \sigma_{2}^{2} Y_{\infty}+\sigma_{3}^{2} & -\left(\sigma_{2}^{2} Y_{\infty}+\sigma_{3}^{2}\right) Y_{\infty} & -\left(\sigma_{2}^{2} Y_{\infty}+\sigma_{3}^{2}\right) X_{\infty} \\
-\varrho \sigma_{1} \sigma_{2} Y_{\infty}^{2} & \varrho \sigma_{1} \sigma_{2} Y_{\infty}^{3} & -\left(\sigma_{2}^{2} Y_{\infty}+\sigma_{3}^{2}\right) Y_{\infty} & \left(\sigma_{2}^{2} Y_{\infty}+\sigma_{3}^{2}\right) Y_{\infty}^{2} & \left(\sigma_{2}^{2} Y_{\infty}+\sigma_{3}^{2}\right) Y_{\infty} X_{\infty} \\
-\varrho \sigma_{1} \sigma_{2} Y_{\infty} X_{\infty} & \varrho \sigma_{1} \sigma_{2} Y_{\infty}^{2} X_{\infty} & -\left(\sigma_{2}^{2} Y_{\infty}+\sigma_{3}^{2}\right) X_{\infty} & \left(\sigma_{2}^{2} Y_{\infty}+\sigma_{3}^{2}\right) Y_{\infty} X_{\infty} & \left(\sigma_{2}^{2} Y_{\infty}+\sigma_{3}^{2}\right) X_{\infty}^{2}
\end{array}\right]
$$

where the random vector $\left(Y_{\infty}, X_{\infty}\right)$ is given by Theorem $A .1$.

Proof. By (3.7), we have

$$
T^{\frac{1}{2}}\left(\widehat{\boldsymbol{\theta}}_{T}-\boldsymbol{\theta}\right)=\left(T^{-1} \boldsymbol{G}_{T}\right)^{-1}\left(T^{-\frac{1}{2}} \boldsymbol{h}_{T}\right)
$$

on the event where $\boldsymbol{G}_{T}$ is invertible, which holds almost surely, see Lemma 3.3. By (4.4), we have $\left(T^{-1} \boldsymbol{G}_{T}\right)^{-1} \stackrel{\text { a.s. }}{\longrightarrow}\left[\mathbb{E}\left(\boldsymbol{G}_{\infty}\right)\right]^{-1}$ as $T \rightarrow \infty$. The process $\left(\boldsymbol{h}_{t}\right)_{t \in \mathbb{R}_{+}}$is a 5 -dimensional continuous local martingale with quadratic variation process $\langle\boldsymbol{h}\rangle_{t}=\widetilde{\boldsymbol{G}}_{t}, \quad t \in \mathbb{R}_{+}$, where

$$
\widetilde{\boldsymbol{G}}_{t}:=\int_{0}^{t}\left[\begin{array}{ccccc}
\sigma_{1}^{2} Y_{s} & -\sigma_{1}^{2} Y_{s}^{2} & \varrho \sigma_{1} \sigma_{2} Y_{s} & -\varrho \sigma_{1} \sigma_{2} Y_{s}^{2} & -\varrho \sigma_{1} \sigma_{2} Y_{s} X_{s} \\
-\sigma_{1}^{2} Y_{s}^{2} & \sigma_{1}^{2} Y_{s}^{3} & -\varrho \sigma_{1} \sigma_{2} Y_{s}^{2} & \varrho \sigma_{1} \sigma_{2} Y_{s}^{3} & \varrho \sigma_{1} \sigma_{2} Y_{s}^{2} X_{s} \\
\varrho \sigma_{1} \sigma_{2} Y_{s} & -\varrho \sigma_{1} \sigma_{2} Y_{s}^{2} & \sigma_{2}^{2} Y_{s}+\sigma_{3}^{2} & -\left(\sigma_{2}^{2} Y_{s}+\sigma_{3}^{2}\right) Y_{s} & -\left(\sigma_{2}^{2} Y_{s}+\sigma_{3}^{2}\right) X_{s} \\
-\varrho \sigma_{1} \sigma_{2} Y_{s}^{2} & \varrho \sigma_{1} \sigma_{2} Y_{s}^{3} & -\left(\sigma_{2}^{2} Y_{s}+\sigma_{3}^{2}\right) Y_{s} & \left(\sigma_{2}^{2} Y_{s}+\sigma_{3}^{2}\right) Y_{s}^{2} & \left(\sigma_{2}^{2} Y_{s}+\sigma_{3}^{2}\right) Y_{s} X_{s} \\
-\varrho \sigma_{1} \sigma_{2} Y_{s} X_{s} & \varrho \sigma_{1} \sigma_{2} Y_{s}^{2} X_{s} & -\left(\sigma_{2}^{2} Y_{s}+\sigma_{3}^{2}\right) X_{s} & \left(\sigma_{2}^{2} Y_{s}+\sigma_{3}^{2}\right) Y_{s} X_{s} & \left(\sigma_{2}^{2} Y_{s}+\sigma_{3}^{2}\right) X_{s}^{2}
\end{array}\right]
$$

By Theorem A.2, we obtain

$$
T^{-1} \widetilde{\boldsymbol{G}}_{T} \stackrel{\text { a.s. }}{\longrightarrow} \mathbb{E}\left(\widetilde{\boldsymbol{G}}_{\infty}\right) \quad \text { as } T \rightarrow \infty
$$


since, by Theorem B.2, the entries of $\mathbb{E}\left(\widetilde{\boldsymbol{G}}_{\infty}\right)$ exist and finite. Using (15.3), Theorem D.2 yields $T^{-\frac{1}{2}} \boldsymbol{h}_{T} \stackrel{\mathcal{D}}{\longrightarrow} \mathcal{N}_{5}\left(\mathbf{0}, \mathbb{E}\left(\widetilde{\boldsymbol{G}}_{\infty}\right)\right)$ as $T \rightarrow \infty$. Hence, by (15.2) and by Slutsky's lemma,

$$
T^{\frac{1}{2}}\left(\widehat{\boldsymbol{\theta}}_{T}-\boldsymbol{\theta}\right) \stackrel{\mathcal{D}}{\longrightarrow}\left[\mathbb{E}\left(\boldsymbol{G}_{\infty}\right)\right]^{-1} \mathcal{N}_{5}\left(\mathbf{0}, \mathbb{E}\left(\widetilde{\boldsymbol{G}}_{\infty}\right)\right)=\mathcal{N}_{5}\left(\mathbf{0},\left[\mathbb{E}\left(\boldsymbol{G}_{\infty}\right)\right]^{-1} \mathbb{E}\left(\widetilde{\boldsymbol{G}}_{\infty}\right)\left(\left[\mathbb{E}\left(\boldsymbol{G}_{\infty}\right)\right]^{-1}\right)^{\top}\right)
$$

as $T \rightarrow \infty$.

\section{Asymptotic behavior of CLSE: critical case}

First we present an auxiliary lemma.

6.1 Lemma. If $\left(\mathcal{Y}_{t}, \mathcal{X}_{t}\right)_{t \in \mathbb{R}_{+}}$and $\left(\widetilde{\mathcal{Y}}_{t}, \widetilde{\mathcal{X}}_{t}\right)_{t \in \mathbb{R}_{+}}$are continuous semimartingales with $\left(\mathcal{Y}_{t}, \mathcal{X}_{t}\right)_{t \in \mathbb{R}_{+}} \stackrel{\mathcal{D}}{=}$ $\left(\widetilde{\mathcal{Y}}_{t}, \widetilde{\mathcal{X}}_{t}\right)_{t \in \mathbb{R}_{+}}$, then

$$
\begin{aligned}
& \left(\mathcal{Y}_{1}, \mathcal{X}_{1}, \int_{0}^{1} \mathcal{X}_{s} \mathrm{~d} \mathcal{Y}_{s}, \int_{0}^{1} \mathcal{Y}_{s}^{k} \mathcal{X}_{s}^{\ell} \mathrm{d} s: k, \ell \in \mathbb{Z}_{+}, k+\ell \leqslant n\right) \\
& \stackrel{\mathcal{D}}{=}\left(\widetilde{\mathcal{Y}}_{1}, \widetilde{\mathcal{X}}_{1}, \int_{0}^{1} \widetilde{\mathcal{X}}_{s} \mathrm{~d} \widetilde{\mathcal{Y}}_{s}, \int_{0}^{1} \widetilde{\mathcal{Y}}_{s}^{k} \widetilde{\mathcal{X}}_{s}^{\ell} \mathrm{d} s: k, \ell \in \mathbb{Z}_{+}, k+\ell \leqslant n\right)
\end{aligned}
$$

for each $n \in \mathbb{N}$.

Proof. By Proposition I.4.44 in Jacod and Shiryaev 23] with the Riemann sequence of deterministic subdivisions $\left(\frac{i}{n} \wedge T\right)_{i \in \mathbb{N}}, n \in \mathbb{N}$, we have

$$
\sum_{i=1}^{n} \mathcal{X}_{\frac{i-1}{n}}\left(\mathcal{Y}_{\frac{i}{n}}-\mathcal{Y}_{\frac{i-1}{n}}\right) \stackrel{\mathbb{P}}{\longrightarrow} \int_{0}^{1} \mathcal{X}_{s} \mathrm{~d} \mathcal{Y}_{s}, \quad \frac{1}{n} \sum_{i=1}^{n} \mathcal{Y}_{\frac{i}{n}}^{k} \mathcal{X}_{\frac{i}{n}}^{\ell} \stackrel{\mathbb{P}}{\longrightarrow} \int_{0}^{1} \mathcal{Y}_{s}^{k} \mathcal{X}_{s}^{\ell} \mathrm{d} s
$$

as $n \rightarrow \infty$ for each $k, \ell \in \mathbb{Z}_{+}$, and similar convergences hold for $\left(\widetilde{\mathcal{Y}}_{t}, \widetilde{\mathcal{X}}_{t}\right)_{t \in \mathbb{R}_{+}}$. The assumption implies

$$
\sum_{i=1}^{n} \mathcal{X}_{\frac{i-1}{n}}\left(\mathcal{Y}_{\frac{i}{n}}-\mathcal{Y}_{\frac{i-1}{n}}\right) \stackrel{\mathcal{D}}{=} \sum_{i=1}^{n} \widetilde{\mathcal{X}}_{\frac{i-1}{n}}\left(\widetilde{\mathcal{Y}}_{\frac{i}{n}}-\widetilde{\mathcal{Y}}_{\frac{i-1}{n}}\right), \quad \frac{1}{n} \sum_{i=1}^{n} \mathcal{Y}_{\frac{i}{n}}^{k} \mathcal{X}_{\frac{i}{n}}^{\ell} \stackrel{\mathcal{D}}{=} \frac{1}{n} \sum_{i=1}^{n} \widetilde{\mathcal{Y}}_{\frac{i}{n}}^{k} \widetilde{\mathcal{X}}_{\frac{i}{n}}^{\ell}
$$

for each $n \in \mathbb{N}$ and $k, \ell \in \mathbb{Z}_{+}$, hence we obtain the statement.

6.2 Theorem. Let us consider the two-factor affine diffusion model (1.1) with $a \in \mathbb{R}_{+}, \quad b=0$, $\alpha \in \mathbb{R}, \quad \beta=0, \quad \gamma=0, \quad \sigma_{1}, \sigma_{2}, \sigma_{3} \in \mathbb{R}_{+}$and $\varrho \in[-1,1]$ with a random initial value $\left(\eta_{0}, \zeta_{0}\right)$ independent of $\left(W_{t}, B_{t}, L_{t}\right)_{t \in \mathbb{R}_{+}}$satisfying $\mathbb{P}\left(\eta_{0} \in \mathbb{R}_{+}\right)=1$. Suppose that $\left(1-\varrho^{2}\right) \sigma_{2}^{2}+\sigma_{3}^{2}>0$. Then

$$
\left[\begin{array}{c}
\widehat{a}_{T}-a \\
T \widehat{b}_{T} \\
\widehat{\alpha}_{T}-\alpha \\
T \widehat{\beta}_{T} \\
T \widehat{\gamma}_{T}
\end{array}\right] \stackrel{\mathcal{D}}{\longrightarrow}\left[\left(\begin{array}{c}
\left.\int_{0}^{1}\left[\begin{array}{c}
1 \\
-\mathcal{Y}_{s}
\end{array}\right]\left[\begin{array}{c}
1 \\
-\mathcal{Y}_{s}
\end{array}\right]^{\top} \mathrm{d} s\right)^{-1}\left[\begin{array}{c}
\mathcal{Y}_{1}-a \\
-\frac{1}{2} \mathcal{Y}_{1}^{2}+\left(a+\frac{\sigma_{1}^{2}}{2}\right) \int_{0}^{1} \mathcal{Y}_{s} \mathrm{~d} s
\end{array}\right] \\
\left.\left.\int_{0}^{1}\left[\begin{array}{c}
1 \\
-\mathcal{Y}_{s} \\
-\mathcal{X}_{s}
\end{array}\right]\left[\begin{array}{c}
1 \\
-\mathcal{Y}_{s} \\
-\mathcal{X}_{s}
\end{array}\right]^{\top}\right)^{-1} \mathrm{~d} s\right)^{-\alpha}\left[\begin{array}{c}
\mathcal{Y}_{1} \mathcal{X}_{1}+\left(\alpha+\varrho \sigma_{1} \sigma_{2}\right) \int_{0}^{1} \mathcal{Y}_{s} \mathrm{~d} s+\int_{0}^{1} \mathcal{X}_{s} \mathrm{~d} \mathcal{Y}_{s} \\
-\frac{1}{2} \mathcal{X}_{1}^{2}+\alpha \int_{0}^{1} \mathcal{X}_{s} \mathrm{~d} s+\frac{\sigma_{2}^{2}}{2} \int_{0}^{1} \mathcal{Y}_{s} \mathrm{~d} s+\frac{\sigma_{3}^{2}}{2}
\end{array}\right]
\end{array}\right]\right.
$$


as $T \rightarrow \infty$, where $\left(\mathcal{Y}_{t}, \mathcal{X}_{t}\right)_{t \in \mathbb{R}_{+}}$is the unique strong solution of the SDE

$$
\left\{\begin{array}{l}
\mathrm{d} \mathcal{Y}_{t}=a \mathrm{~d} t+\sigma_{1} \sqrt{\mathcal{Y}_{t}} \mathrm{~d} W_{t}, \\
\mathrm{~d} \mathcal{X}_{t}=\alpha \mathrm{d} t+\sigma_{2} \sqrt{\mathcal{Y}_{t}}\left(\varrho \mathrm{d} W_{t}+\sqrt{1-\varrho^{2}} \mathrm{~d} B_{t}\right),
\end{array} \quad t \in[0, \infty),\right.
$$

with initial value $\left(\mathcal{Y}_{0}, \mathcal{X}_{0}\right)=(0,0)$.

Proof. By (3.7), we have

$$
\left[\begin{array}{c}
\widehat{a}_{T}-a \\
\widehat{b}_{T}
\end{array}\right]=\left[\begin{array}{c}
\widehat{a}_{T}-a \\
\widehat{b}_{T}-b
\end{array}\right]=\left[\begin{array}{cc}
T & -\int_{0}^{T} Y_{s} \mathrm{~d} s \\
-\int_{0}^{T} Y_{s} \mathrm{~d} s & \int_{0}^{T} Y_{s}^{2} \mathrm{~d} s
\end{array}\right]^{-1}\left[\begin{array}{c}
\sigma_{1} \int_{0}^{T} Y_{s}^{\frac{1}{2}} \mathrm{~d} W_{s} \\
-\sigma_{1} \int_{0}^{T} Y_{s}^{\frac{3}{2}} \mathrm{~d} W_{s}
\end{array}\right] .
$$

We can write

$$
\left[\begin{array}{cc}
T & -\int_{0}^{T} Y_{s} \mathrm{~d} s \\
-\int_{0}^{T} Y_{s} \mathrm{~d} s & \int_{0}^{T} Y_{s}^{2} \mathrm{~d} s
\end{array}\right]=\left[\begin{array}{cc}
T^{\frac{1}{2}} & 0 \\
0 & T^{\frac{3}{2}}
\end{array}\right]\left[\begin{array}{cc}
1 & -\frac{1}{T^{2}} \int_{0}^{T} Y_{s} \mathrm{~d} s \\
-\frac{1}{T^{2}} \int_{0}^{T} Y_{s} \mathrm{~d} s & \frac{1}{T^{3}} \int_{0}^{T} Y_{s}^{2} \mathrm{~d} s
\end{array}\right]\left[\begin{array}{cc}
T^{\frac{1}{2}} & 0 \\
0 & T^{\frac{3}{2}}
\end{array}\right]
$$

and

$$
\left[\begin{array}{c}
\sigma_{1} \int_{0}^{T} Y_{s}^{\frac{1}{2}} \mathrm{~d} W_{s} \\
-\sigma_{1} \int_{0}^{T} Y_{s}^{\frac{3}{2}} \mathrm{~d} W_{s}
\end{array}\right]=\left[\begin{array}{cc}
T & 0 \\
0 & T^{2}
\end{array}\right]\left[\begin{array}{c}
\frac{\sigma_{1}}{T} \int_{0}^{T} Y_{s}^{\frac{1}{2}} \mathrm{~d} W_{s} \\
-\frac{\sigma_{1}}{T^{2}} \int_{0}^{T} Y_{s}^{\frac{3}{2}} \mathrm{~d} W_{s}
\end{array}\right]
$$

Consequently,

$$
\left[\begin{array}{c}
\widehat{a}_{T}-a \\
T \widehat{b}_{T}
\end{array}\right]=\left[\begin{array}{cc}
1 & 0 \\
0 & T
\end{array}\right]\left[\begin{array}{c}
\widehat{a}_{T}-a \\
\widehat{b}_{T}
\end{array}\right]=\left[\begin{array}{cc}
1 & -\frac{1}{T^{2}} \int_{0}^{T} Y_{s} \mathrm{~d} s \\
-\frac{1}{T^{2}} \int_{0}^{T} Y_{s} \mathrm{~d} s & \frac{1}{T^{3}} \int_{0}^{T} Y_{s}^{2} \mathrm{~d} s
\end{array}\right]^{-1}\left[\begin{array}{c}
\frac{\sigma_{1}}{T} \int_{0}^{T} Y_{s}^{\frac{1}{2}} \mathrm{~d} W_{s} \\
-\frac{\sigma_{1}}{T^{2}} \int_{0}^{T} Y_{s}^{\frac{3}{2}} \mathrm{~d} W_{s}
\end{array}\right] .
$$

In a similar way,

$$
\begin{aligned}
{\left[\begin{array}{c}
\widehat{\alpha}_{T}-\alpha \\
T \widehat{\beta}_{T} \\
T \widehat{\gamma}_{T}
\end{array}\right]=} & {\left[\begin{array}{ccc}
1 & -\frac{1}{T^{2}} \int_{0}^{T} Y_{s} \mathrm{~d} s & -\frac{1}{T^{2}} \int_{0}^{T} X_{s} \mathrm{~d} s \\
-\frac{1}{T^{2}} \int_{0}^{T} Y_{s} \mathrm{~d} s & \frac{1}{T^{3}} \int_{0}^{T} Y_{s}^{2} \mathrm{~d} s & \frac{1}{T^{3}} \int_{0}^{T} Y_{s} X_{s} \mathrm{~d} s \\
-\frac{1}{T^{2}} \int_{0}^{T} X_{s} \mathrm{~d} s & \frac{1}{T^{3}} \int_{0}^{T} Y_{s} X_{s} \mathrm{~d} s & \frac{1}{T^{3}} \int_{0}^{T} X_{s}^{2} \mathrm{~d} s
\end{array}\right]^{-1} } \\
\times & {\left[\begin{array}{c}
\frac{\sigma_{2}}{T} \int_{0}^{T} Y_{s}^{\frac{1}{2}} \mathrm{~d} \widetilde{W}_{s}+\frac{\sigma_{3}}{T} L_{T} \\
-\frac{\sigma_{2}}{T^{2}} \int_{0}^{T} Y_{s}^{\frac{3}{2}} \mathrm{~d} \widetilde{W}_{s}-\frac{\sigma_{3}}{T^{2}} \int_{0}^{T} Y_{s} \mathrm{~d} L_{s} \\
-\frac{\sigma_{2}}{T^{2}} \int_{0}^{T} Y_{s}^{\frac{1}{2}} X_{s} \mathrm{~d} \widetilde{W}_{s}-\frac{\sigma_{3}}{T^{2}} \int_{0}^{T} X_{s} \mathrm{~d} L_{s}
\end{array}\right] }
\end{aligned}
$$

The aim of the following discussion is to prove

$$
\begin{aligned}
& \left(\frac{1}{T} Y_{T}, \frac{1}{T} X_{T}, \frac{1}{T^{2}} \int_{0}^{T} X_{s} \mathrm{~d} Y_{s}, \frac{1}{T^{k+\ell+1}} \int_{0}^{T} Y_{s}^{k} X_{s}^{\ell} \mathrm{d} s: k, \ell \in \mathbb{Z}_{+}, k+\ell \leqslant 2\right) \\
& \stackrel{\mathcal{D}}{\longrightarrow}\left(\mathcal{Y}_{1}, \mathcal{X}_{1}, \int_{0}^{1} \mathcal{X}_{s} \mathrm{~d} \mathcal{Y}_{s}, \int_{0}^{1} \mathcal{Y}_{s}^{k} \mathcal{X}_{s}^{\ell} \mathrm{d} s: k, \ell \in \mathbb{Z}_{+}, k+\ell \leqslant 2\right)
\end{aligned}
$$

as $T \rightarrow \infty$. By part (ii) of Remark 2.7 in Barczy et al. [5], we have

$$
\left(\widetilde{\mathcal{Y}}_{t}^{(T)}, \widetilde{\mathcal{X}}_{t}^{(T)}\right)_{t \in \mathbb{R}_{+}}:=\left(\frac{1}{T} \mathcal{Y}_{T t}, \frac{1}{T} \mathcal{X}_{T t}\right)_{t \in \mathbb{R}_{+}} \stackrel{\mathcal{D}}{=}\left(\mathcal{Y}_{t}, \mathcal{X}_{t}\right)_{t \in \mathbb{R}_{+}} \quad \text { for all } T \in \mathbb{R}_{++},
$$


since, by Proposition 2.1, $\left(\mathcal{Y}_{t}, \mathcal{X}_{t}\right)_{t \in \mathbb{R}_{+}}$is an affine process with infinitesimal generator

$$
\left(\mathcal{A}_{(\mathcal{Y}, \mathcal{X})} f\right)(y, x)=a f_{1}^{\prime}(y, x)+\alpha f_{2}^{\prime}(y, x)+\frac{1}{2} y\left[\sigma_{1}^{2} f_{1,1}^{\prime \prime}(y, x)+2 \varrho \sigma_{1} \sigma_{2} f_{1,2}^{\prime \prime}(y, x)+\sigma_{2}^{2} f_{2,2}^{\prime \prime}(y, x)\right] .
$$

Hence, by Lemma 6.1, we obtain

$$
\begin{aligned}
& \left(\mathcal{Y}_{1}, \mathcal{X}_{1}, \int_{0}^{1} \mathcal{X}_{s} \mathrm{~d} \mathcal{Y}_{s}, \int_{0}^{1} \mathcal{Y}_{s}^{k} \mathcal{X}_{s}^{\ell} \mathrm{d} s: k, \ell \in \mathbb{Z}_{+}, k+\ell \leqslant 2\right) \\
& \stackrel{\mathcal{D}}{=}\left(\widetilde{\mathcal{Y}}_{1}^{(T)}, \widetilde{\mathcal{X}}_{1}^{(T)}, \int_{0}^{1} \widetilde{\mathcal{X}}_{s}^{(T)} \mathrm{d} \widetilde{\mathcal{Y}}_{s}^{(T)}, \int_{0}^{1}\left(\widetilde{\mathcal{Y}}_{s}^{(T)}\right)^{k}\left(\widetilde{\mathcal{X}}_{s}^{(T)}\right)^{\ell} \mathrm{d} s: k, \ell \in \mathbb{Z}_{+}, k+\ell \leqslant 2\right) \\
& =\left(\frac{1}{T} \mathcal{Y}_{T}, \frac{1}{T} \mathcal{X}_{T}, \frac{1}{T^{2}} \int_{0}^{T} \mathcal{X}_{s} \mathrm{~d} \mathcal{Y}_{s}, \frac{1}{T^{k+\ell+1}} \int_{0}^{T} \mathcal{Y}_{s}^{k} \mathcal{X}_{s}^{\ell} \mathrm{d} s: k, \ell \in \mathbb{Z}_{+}, k+\ell \leqslant 2\right)
\end{aligned}
$$

for all $T \in \mathbb{R}_{++}$. Then, by Slutsky's lemma, in order to prove (6.3), it suffices to show the convergences

$$
\begin{aligned}
& \frac{1}{T}\left(Y_{T}-\mathcal{Y}_{T}\right) \stackrel{\mathbb{P}}{\longrightarrow} 0, \quad \frac{1}{T}\left(X_{T}-\mathcal{X}_{T}\right) \stackrel{\mathbb{P}}{\longrightarrow} 0, \\
& \frac{1}{T^{2}}\left(\int_{0}^{T} X_{s} \mathrm{~d} Y_{s}-\int_{0}^{T} \mathcal{X}_{s} \mathrm{~d} \mathcal{Y}_{s}\right) \stackrel{\mathbb{P}}{\longrightarrow} 0, \quad \frac{1}{T^{k+\ell+1}} \int_{0}^{T}\left(Y_{s}^{k} X_{s}^{\ell}-\mathcal{Y}_{s}^{k} \mathcal{X}_{s}^{\ell}\right) \mathrm{d} s \stackrel{\mathbb{P}}{\longrightarrow} 0
\end{aligned}
$$

as $T \rightarrow \infty$ for all $k, \ell \in \mathbb{Z}_{+}$with $k+\ell \leqslant 2$. By (3.21) in Barczy et al. [5], we have

$$
\mathbb{E}\left(\left|Y_{s}-\mathcal{Y}_{s}\right|\right) \leqslant \mathbb{E}\left(Y_{0}\right), \quad s \in \mathbb{R}_{+},
$$

hence

$$
\begin{aligned}
& \mathbb{E}\left(\left|\frac{1}{T}\left(Y_{T}-\mathcal{Y}_{T}\right)\right|\right) \leqslant \frac{1}{T} \mathbb{E}\left(Y_{0}\right) \rightarrow 0 \\
& \mathbb{E}\left(\left|\frac{1}{T^{2}} \int_{0}^{T}\left(Y_{s}-\mathcal{Y}_{s}\right) \mathrm{d} s\right|\right) \leqslant \frac{1}{T^{2}} \int_{0}^{T} \mathbb{E}\left(\left|Y_{s}-\mathcal{Y}_{s}\right|\right) \mathrm{d} s \leqslant \frac{1}{T} \mathbb{E}\left(Y_{0}\right) \rightarrow 0,
\end{aligned}
$$

as $T \rightarrow \infty$, implying $\frac{1}{T}\left(Y_{T}-\mathcal{Y}_{T}\right) \stackrel{\mathbb{P}}{\longrightarrow} 0$ and $\frac{1}{T^{2}} \int_{0}^{T}\left(Y_{s}-\mathcal{Y}_{s}\right) \mathrm{d} s \stackrel{\mathbb{P}}{\longrightarrow} 0$ as $T \rightarrow \infty$, i.e., the first convergence in (6.4) and the second convergence in (6.5) for $(k, \ell)=(1,0)$.

As in (3.23) in Barczy et al. [5], we have $\mathbb{E}\left(\left|X_{s}-\mathcal{X}_{s}\right|\right) \leqslant \mathbb{E}\left(\left|X_{0}\right|\right)+\sqrt{\left(\sigma_{2}^{2} \mathbb{E}\left(Y_{0}\right)+\sigma_{3}^{2}\right) s}$ for all $s \in \mathbb{R}_{+}$, hence

$$
\sup _{s \in[0, T]} \mathbb{E}\left(\left|X_{s}-\mathcal{X}_{s}\right|\right)=\mathrm{O}\left(T^{\frac{1}{2}}\right) \quad \text { as } T \rightarrow \infty
$$

thus

$$
\begin{aligned}
& \mathbb{E}\left(\left|\frac{1}{T}\left(X_{T}-\mathcal{X}_{T}\right)\right|\right)=\frac{1}{T} \mathrm{O}\left(T^{\frac{1}{2}}\right) \rightarrow 0 \\
& \mathbb{E}\left(\left|\frac{1}{T^{2}} \int_{0}^{T}\left(X_{s}-\mathcal{X}_{s}\right) \mathrm{d} s\right|\right) \leqslant \frac{1}{T^{2}} \int_{0}^{T} \mathbb{E}\left(\left|X_{s}-\mathcal{X}_{s}\right|\right) \mathrm{d} s=\frac{1}{T^{2}} \int_{0}^{T} \mathrm{O}\left(T^{\frac{1}{2}}\right) \mathrm{d} s=\frac{1}{T^{2}} \mathrm{O}\left(T^{\frac{3}{2}}\right) \rightarrow 0,
\end{aligned}
$$

as $T \rightarrow \infty$, implying $\frac{1}{T}\left(X_{T}-\mathcal{X}_{T}\right) \stackrel{\mathbb{P}}{\longrightarrow} 0$ and $\frac{1}{T^{2}} \int_{0}^{T}\left(X_{s}-\mathcal{X}_{s}\right) \mathrm{d} s \stackrel{\mathbb{P}}{\longrightarrow} 0$ as $T \rightarrow \infty$, i.e., the second convergence in (6.4) and the second convergence in (6.5) for $(k, \ell)=(0,1)$. 
As in (3.25) in Barczy et al. [5], we have $\mathbb{E}\left[\left(Y_{s}-\mathcal{Y}_{s}\right)^{2}\right] \leqslant 2 \mathbb{E}\left(Y_{0}^{2}\right)+2 s \sigma_{1}^{2} \mathbb{E}\left(Y_{0}\right)$ for all $s \in \mathbb{R}_{+}$, hence

$$
\sup _{s \in[0, T]} \mathbb{E}\left[\left(Y_{s}-\mathcal{Y}_{s}\right)^{2}\right]=\mathrm{O}(T) \quad \text { as } T \rightarrow \infty
$$

By Proposition B.1, $\mathbb{E}\left(Y_{s}^{2}\right)=\mathbb{E}\left(Y_{0}^{2}\right)+\left(2 a+\sigma_{1}^{2}\right)\left(\mathbb{E}\left(Y_{0}\right) s+a \frac{s^{2}}{2}\right)$ for all $s \in \mathbb{R}_{+}$, hence

$$
\sup _{s \in[0, T]} \mathbb{E}\left(Y_{s}^{2}\right)=\mathrm{O}\left(T^{2}\right) \quad \text { as } T \rightarrow \infty
$$

and $\sup _{s \in[0, T]} \mathbb{E}\left(\mathcal{Y}_{s}^{2}\right)=\mathrm{O}\left(T^{2}\right)$ as $T \rightarrow \infty$. We have

$$
\begin{aligned}
\mathbb{E}\left(\left|Y_{s}^{2}-\mathcal{Y}_{s}^{2}\right|\right)=\mathbb{E}\left(\left|\left(Y_{s}-\mathcal{Y}_{s}\right)\left(Y_{s}+\mathcal{Y}_{s}\right)\right|\right) & \leqslant \sqrt{\mathbb{E}\left[\left(Y_{s}-\mathcal{Y}_{s}\right)^{2}\right] \mathbb{E}\left[\left(Y_{s}+\mathcal{Y}_{s}\right)^{2}\right]} \\
& \leqslant \sqrt{2 \mathbb{E}\left[\left(Y_{s}-\mathcal{Y}_{s}\right)^{2}\right]\left(\mathbb{E}\left(Y_{s}^{2}\right)+\mathbb{E}_{\left.\left(\mathcal{Y}_{s}^{2}\right)\right)}\right.}
\end{aligned}
$$

yielding

$$
\sup _{s \in[0, T]} \mathbb{E}\left(\left|Y_{s}^{2}-\mathcal{Y}_{s}^{2}\right|\right)=\sqrt{2 \mathrm{O}(T)\left(\mathrm{O}\left(T^{2}\right)+\mathrm{O}\left(T^{2}\right)\right)}=\mathrm{O}\left(T^{\frac{3}{2}}\right) \quad \text { as } \quad T \rightarrow \infty
$$

thus

$$
\mathbb{E}\left(\left|\frac{1}{T^{3}} \int_{0}^{T}\left(Y_{s}^{2}-\mathcal{Y}_{s}^{2}\right) \mathrm{d} s\right|\right) \leqslant \frac{1}{T^{3}} \int_{0}^{T} \mathbb{E}\left(\left|Y_{s}^{2}-\mathcal{Y}_{s}^{2}\right|\right) \mathrm{d} s=\frac{1}{T^{3}} \int_{0}^{T} \mathrm{O}\left(T^{\frac{3}{2}}\right) \mathrm{d} s=\frac{1}{T^{3}} \mathrm{O}\left(T^{\frac{5}{2}}\right) \rightarrow 0,
$$

as $T \rightarrow \infty$, implying $\frac{1}{T^{3}} \int_{0}^{T}\left(Y_{s}^{2}-\mathcal{Y}_{s}^{2}\right) \mathrm{d} s \stackrel{\mathbb{P}}{\longrightarrow} 0$ as $T \rightarrow \infty$., i.e., the second convergence in (6.5) for $(k, \ell)=(2,0)$.

In a similar way, $\mathbb{E}\left[\left(X_{s}-\mathcal{X}_{s}\right)^{2}\right] \leqslant 2 \mathbb{E}\left(X_{0}^{2}\right)+2 s\left(\sigma_{2}^{2} \mathbb{E}\left(Y_{0}\right)+\sigma_{3}^{2}\right)$ for all $s \in \mathbb{R}_{+}$, hence

$$
\sup _{s \in[0, T]} \mathbb{E}\left[\left(X_{s}-\mathcal{X}_{s}\right)^{2}\right]=\mathrm{O}(T) \quad \text { as } T \rightarrow \infty .
$$

By Proposition B.1, $\mathbb{E}\left(X_{s}^{2}\right)=\mathbb{E}\left(X_{0}^{2}\right)+\alpha\left(s \mathbb{E}\left(X_{0}\right)+\alpha \frac{s^{2}}{2}\right)+\sigma_{2}^{2}\left(s \mathbb{E}\left(Y_{0}\right)+a \frac{s^{2}}{2}\right)+\sigma_{3}^{2} s$, thus $\sup _{s \in[0, T]} \mathbb{E}\left(X_{s}^{2}\right)=\mathrm{O}\left(T^{2}\right)$ and $\sup _{s \in[0, T]} \mathbb{E}\left(\mathcal{X}_{s}^{2}\right)=\mathrm{O}\left(T^{2}\right)$ as $T \rightarrow \infty$. We have

$$
\mathbb{E}\left(\left|X_{s}^{2}-\mathcal{X}_{s}^{2}\right|\right) \leqslant \sqrt{2 \mathbb{E}\left[\left(X_{s}-\mathcal{X}_{s}\right)^{2}\right]\left(\mathbb{E}\left(X_{s}^{2}\right)+\mathbb{E}\left(\mathcal{X}_{s}^{2}\right)\right)},
$$

yielding

$$
\sup _{s \in[0, T]} \mathbb{E}\left(\left|X_{s}^{2}-\mathcal{X}_{s}^{2}\right|\right)=\sqrt{2 \mathrm{O}(T)\left(\mathrm{O}\left(T^{2}\right)+\mathrm{O}\left(T^{2}\right)\right)}=\mathrm{O}\left(T^{\frac{3}{2}}\right) \quad \text { as } \quad T \rightarrow \infty
$$

thus

$$
\mathbb{E}\left(\left|\frac{1}{T^{3}} \int_{0}^{T}\left(X_{s}^{2}-\mathcal{X}_{s}^{2}\right) \mathrm{d} s\right|\right) \leqslant \frac{1}{T^{3}} \int_{0}^{T} \mathbb{E}\left(\left|X_{s}^{2}-\mathcal{X}_{s}^{2}\right|\right) \mathrm{d} s=\frac{1}{T^{3}} \int_{0}^{T} \mathrm{O}\left(T^{\frac{3}{2}}\right) \mathrm{d} s=\frac{1}{T^{3}} \mathrm{O}\left(T^{\frac{5}{2}}\right) \rightarrow 0,
$$

as $T \rightarrow \infty$, implying $\frac{1}{T^{3}} \int_{0}^{T}\left(X_{s}^{2}-\mathcal{X}_{s}^{2}\right) \mathrm{d} s \stackrel{\mathbb{P}}{\longrightarrow} 0$ as $T \rightarrow \infty$, i.e., the second convergence in (6.5) for $(k, \ell)=(0,2)$.

Further,

$$
\begin{aligned}
\mathbb{E}\left(\left|Y_{s} X_{s}-\mathcal{Y}_{s} \mathcal{X}_{s}\right|\right) & \leqslant \mathbb{E}\left(\left|Y_{s}-\mathcal{Y}_{s}\right|\left|X_{s}\right|\right)+\mathbb{E}\left(\mathcal{Y}_{s}\left|X_{s}-\mathcal{X}_{s}\right|\right) \\
& \leqslant \sqrt{\mathbb{E}\left[\left(Y_{s}-\mathcal{Y}_{s}\right)^{2}\right] \mathbb{E}\left(X_{s}^{2}\right)}+\sqrt{\mathbb{E}\left(\mathcal{Y}_{s}^{2}\right) \mathbb{E}\left[\left(X_{s}-\mathcal{X}_{s}\right)^{2}\right]}
\end{aligned}
$$


yields

$$
\sup _{s \in[0, T]} \mathbb{E}\left(\left|Y_{s} X_{s}-\mathcal{Y}_{s} \mathcal{X}_{s}\right|\right)=\sqrt{\mathrm{O}(T) \mathrm{O}\left(T^{2}\right)}+\sqrt{\left.\mathrm{O}\left(T^{2}\right) \mathrm{O}(T)\right)}=\mathrm{O}\left(T^{\frac{3}{2}}\right) \quad \text { as } \quad T \rightarrow \infty,
$$

thus

$$
\mathbb{E}\left(\left|\frac{1}{T^{3}} \int_{0}^{T}\left(Y_{s} X_{s}-\mathcal{Y}_{s} \mathcal{X}_{s}\right) \mathrm{d} s\right|\right) \leqslant \frac{1}{T^{3}} \int_{0}^{T} \mathbb{E}\left(\left|Y_{s} X_{s}-\mathcal{Y}_{s} \mathcal{X}_{s}\right|\right) \mathrm{d} s=\frac{1}{T^{3}} \int_{0}^{T} \mathrm{O}\left(T^{\frac{3}{2}}\right) \mathrm{d} s=\frac{1}{T^{3}} \mathrm{O}\left(T^{\frac{5}{2}}\right) \rightarrow 0,
$$

as $T \rightarrow \infty$, implying $\frac{1}{T^{3}} \int_{0}^{T}\left(Y_{s} X_{s}-\mathcal{Y}_{s} \mathcal{X}_{s}\right) \mathrm{d} s \stackrel{\mathbb{P}}{\longrightarrow} 0$ as $T \rightarrow \infty$, i.e., the second convergence in (6.5) for $(k, \ell)=(1,1)$.

Using the Cauchy-Schwarz inequality, we obtain

$$
\begin{aligned}
\mathbb{E}\left(\left|\int_{0}^{T} X_{s} \mathrm{~d} Y_{s}-\int_{0}^{T} \mathcal{X}_{s} \mathrm{~d} \mathcal{Y}_{s}\right|\right) & \leqslant \mathbb{E}\left(\left|\int_{0}^{T}\left(X_{s}-\mathcal{X}_{s}\right) \mathrm{d} Y_{s}\right|\right)+\mathbb{E}\left(\left|\int_{0}^{T} \mathcal{X}_{s} \mathrm{~d}\left(Y_{s}-\mathcal{Y}_{s}\right)\right|\right) \\
& \leqslant \sqrt{E_{1}(T)}+\sqrt{E_{2}(T)}
\end{aligned}
$$

with

$$
E_{1}(T):=\mathbb{E}\left(\left|\int_{0}^{T}\left(X_{s}-\mathcal{X}_{s}\right) \mathrm{d} Y_{s}\right|^{2}\right), \quad E_{2}(T):=\mathbb{E}\left(\left|\int_{0}^{T} \mathcal{X}_{s} \mathrm{~d}\left(Y_{s}-\mathcal{Y}_{s}\right)\right|^{2}\right) .
$$

Using $\mathrm{d} Y_{s}=a \mathrm{~d} s+\sigma_{1} \sqrt{Y_{s}} \mathrm{~d} W_{s}$, we have

$$
E_{1}(T)=\mathbb{E}\left(\left|a \int_{0}^{T}\left(X_{s}-\mathcal{X}_{s}\right) \mathrm{d} s+\sigma_{1} \int_{0}^{T}\left(X_{s}-\mathcal{X}_{s}\right) \sqrt{Y_{s}} \mathrm{~d} W_{s}\right|^{2}\right) \leqslant 2 a^{2} E_{1,1}(T)+2 \sigma_{1}^{2} E_{1,2}(T)
$$

with

$$
E_{1,1}(T):=\mathbb{E}\left(\left|\int_{0}^{T}\left(X_{s}-\mathcal{X}_{s}\right) \mathrm{d} s\right|^{2}\right), \quad E_{1,2}(T):=\mathbb{E}\left(\left|\int_{0}^{T}\left(X_{s}-\mathcal{X}_{s}\right) \sqrt{Y_{s}} \mathrm{~d} W_{s}\right|^{2}\right) .
$$

Applying (6.10), we obtain

$$
\begin{aligned}
E_{1,1}(T) & =\mathbb{E}\left(\int_{0}^{T} \int_{0}^{T}\left(X_{s}-\mathcal{X}_{s}\right)\left(X_{u}-\mathcal{X}_{u}\right) \mathrm{d} s \mathrm{~d} u\right)=\int_{0}^{T} \int_{0}^{T} \mathbb{E}\left[\left(X_{s}-\mathcal{X}_{s}\right)\left(X_{u}-\mathcal{X}_{u}\right)\right] \mathrm{d} s \mathrm{~d} u \\
& \leqslant \int_{0}^{T} \int_{0}^{T} \sqrt{\mathbb{E}\left[\left(X_{s}-\mathcal{X}_{s}\right)^{2}\right] \mathbb{E}\left[\left(X_{u}-\mathcal{X}_{u}\right)^{2}\right]} \mathrm{d} s \mathrm{~d} u=\int_{0}^{T} \int_{0}^{T} \sqrt{\mathrm{O}(T) \mathrm{O}(T)} \mathrm{d} s \mathrm{~d} u=\mathrm{O}\left(T^{3}\right) .
\end{aligned}
$$

Again by the Cauchy-Schwarz inequality, we obtain

$$
E_{1,2}(T)=\mathbb{E}\left(\int_{0}^{T}\left(X_{s}-\mathcal{X}_{s}\right)^{2} Y_{s} \mathrm{~d} s\right)=\int_{0}^{T} \mathbb{E}\left[\left(X_{s}-\mathcal{X}_{s}\right)^{2} Y_{s}\right] \mathrm{d} s \leqslant \int_{0}^{T} \sqrt{\mathbb{E}\left[\left(X_{s}-\mathcal{X}_{s}\right)^{4}\right] \mathbb{E}\left(Y_{s}^{2}\right)} \mathrm{d} s .
$$

Using $X_{t}=X_{0}+\sigma_{2} \int_{0}^{t} \sqrt{Y_{s}} \mathrm{~d} \widetilde{W}_{s}+\sigma_{3} L_{t}$ and $\mathcal{X}_{t}=\sigma_{2} \int_{0}^{t} \sqrt{\mathcal{Y}_{s}} \mathrm{~d} \widetilde{W}_{s}$, we get $X_{t}-\mathcal{X}_{t}=X_{0}+\sigma_{2} \int_{0}^{t}\left(\sqrt{Y_{s}}-\right.$ $\left.\sqrt{\mathcal{Y}_{s}}\right) \mathrm{d} \widetilde{W}_{s}+\sigma_{3} L_{t}$, and, applying Minkowski inequality and a martingale moment inequality in Karatzas 
and Shreve [24, 3.3.25], we obtain

$$
\begin{aligned}
\left(\mathbb{E}\left[\left(X_{t}-\mathcal{X}_{t}\right)^{4}\right]\right)^{\frac{1}{4}} & \leqslant\left[\mathbb{E}\left(X_{0}^{4}\right)\right]^{\frac{1}{4}}+\sigma_{2}\left(\mathbb{E}\left[\left(\int_{0}^{t}\left(\sqrt{Y_{s}}-\sqrt{\mathcal{Y}_{s}}\right) \mathrm{d} \widetilde{W}_{s}\right)^{4}\right]\right)^{\frac{1}{4}}+\sigma_{3}\left[\mathbb{E}\left(L_{t}^{4}\right)\right]^{\frac{1}{4}} \\
& \leqslant\left[\mathbb{E}\left(X_{0}^{4}\right)\right]^{\frac{1}{4}}+\sigma_{2}\left((2 \cdot 3)^{2} t \mathbb{E}\left(\int_{0}^{t}\left(\sqrt{Y_{s}}-\sqrt{\mathcal{Y}_{s}}\right)^{4} \mathrm{~d} s\right)\right)^{\frac{1}{4}}+\sigma_{3} \sqrt[4]{3} \sqrt{t} \\
& \leqslant\left[\mathbb{E}\left(X_{0}^{4}\right)\right]^{\frac{1}{4}}+\sigma_{2}\left(36 t \int_{0}^{t} \mathbb{E}\left[\left(Y_{s}-\mathcal{Y}_{s}\right)^{2}\right] \mathrm{d} s\right)^{\frac{1}{4}}+\sigma_{3} \sqrt[4]{3} \sqrt{t} .
\end{aligned}
$$

Applying (6.8), we get

$$
\sup _{t \in[0, T]} \mathbb{E}\left[\left(X_{t}-\mathcal{X}_{t}\right)^{4}\right]=\mathrm{O}\left(T^{3}\right) \quad \text { as } T \rightarrow \infty
$$

which, by (6.9), implies $E_{1,2}(T)=\int_{0}^{T} \sqrt{\mathrm{O}\left(T^{3}\right) \mathrm{O}\left(T^{2}\right)} \mathrm{d} s=\mathrm{O}\left(T^{\frac{7}{2}}\right)$ as $T \rightarrow \infty$. Using $E_{1,1}(T)=$ $\mathrm{O}\left(T^{3}\right)$ as $T \rightarrow \infty$, we conclude $E_{1}(T)=\mathrm{O}\left(T^{3}\right)+\mathrm{O}\left(T^{\frac{7}{2}}\right)=\mathrm{O}\left(T^{\frac{7}{2}}\right)$ as $T \rightarrow \infty$.

Using $\mathrm{d} Y_{s}=a \mathrm{~d} s+\sigma_{1} \sqrt{Y_{s}} \mathrm{~d} W_{s} \quad$ and $\quad \mathrm{d} \mathcal{Y}_{s}=a \mathrm{~d} s+\sigma_{1} \sqrt{\mathcal{Y}_{s}} \mathrm{~d} W_{s}, \quad$ we obtain $\mathrm{d}\left(Y_{t}-\mathcal{Y}_{t}\right)=$ $\sigma_{1}\left(\sqrt{Y_{t}}-\sqrt{\mathcal{Y}_{t}}\right) \mathrm{d} W_{t}$, thus

$$
\begin{aligned}
E_{2}(T)=\sigma_{1}^{2} \mathbb{E}\left(\int_{0}^{T} \mathcal{X}_{s}^{2}\left(\sqrt{Y_{s}}-\sqrt{\mathcal{Y}_{s}}\right)^{2} \mathrm{~d} s\right) & \leqslant \sigma_{1}^{2} \int_{0}^{T} \mathbb{E}\left[\mathcal{X}_{s}^{2}\left|Y_{s}-\mathcal{Y}_{s}\right|\right] \mathrm{d} s \\
& \leqslant \sigma_{1}^{2} \int_{0}^{T} \sqrt{\mathbb{E}\left(\mathcal{X}_{s}^{4}\right) \mathbb{E}\left[\left(Y_{s}-\mathcal{Y}_{s}\right)^{2}\right]} \mathrm{d} s .
\end{aligned}
$$

Using $\mathcal{X}_{t}=\alpha t+\sigma_{2} \int_{0}^{t} \sqrt{\mathcal{Y}_{s}} \mathrm{~d} \widetilde{W}_{s}$, we obtain

$$
\begin{aligned}
{\left[\mathbb{E}\left(\mathcal{X}_{t}^{4}\right)\right]^{\frac{1}{4}} } & \leqslant|\alpha| t+\sigma_{2}\left(\mathbb{E}\left[\left(\int_{0}^{t} \sqrt{\mathcal{Y}_{s}} \mathrm{~d} \widetilde{W}_{s}\right)^{4}\right]\right)^{\frac{1}{4}} \leqslant|\alpha| t+\sigma_{2}\left((2 \cdot 3)^{2} t \mathbb{E}\left(\int_{0}^{t} \mathcal{Y}_{s}^{2} \mathrm{~d} s\right)\right)^{\frac{1}{4}} \\
& =|\alpha| t+\sigma_{2}\left(36 t \int_{0}^{t} a\left(a+\frac{\sigma_{1}^{2}}{2}\right) s^{2} \mathrm{~d} s\right)^{\frac{1}{4}}=\left(|\alpha|+\sigma_{2} \sqrt[4]{6 a\left(2 a+\sigma_{1}^{2}\right)}\right) t,
\end{aligned}
$$

hence we conclude

$$
\sup _{s \in[0, T]} \mathbb{E}\left(\mathcal{X}_{s}^{4}\right)=\mathrm{O}\left(T^{4}\right) \quad \text { as } T \rightarrow \infty
$$

Using (6.8), we obtain $E_{2}(T)=\int_{0}^{T} \sqrt{\mathrm{O}\left(T^{4}\right) \mathrm{O}(T)} \mathrm{d} s=\mathrm{O}\left(T^{\frac{7}{2}}\right)$ as $T \rightarrow \infty$. Hence

$$
\mathbb{E}\left(\left|\frac{1}{T^{2}}\left(\int_{0}^{T} X_{s} \mathrm{~d} Y_{s}-\int_{0}^{T} \mathcal{X}_{s} \mathrm{~d} \mathcal{Y}_{s}\right)\right|\right) \leqslant \frac{1}{T^{2}}\left(\sqrt{E_{1}(T)}+\sqrt{E_{2}(T)}\right)=\frac{1}{T^{2}} \mathrm{O}\left(T^{\frac{7}{4}}\right) \rightarrow 0
$$

as $T \rightarrow \infty$, implying $\frac{1}{T^{2}}\left(\int_{0}^{T} X_{s} \mathrm{~d} Y_{s}-\int_{0}^{T} X_{s} \mathrm{~d} Y_{s}\right) \stackrel{\mathbb{P}}{\longrightarrow} 0$ as $T \rightarrow \infty$, i.e., the first convergence in (6.5). Thus we conclude convergence (6.3).

Applying the first equation of (1.1) and using $b=0$, we obtain

$$
\frac{\sigma_{1}}{T} \int_{0}^{T} Y_{s}^{\frac{1}{2}} \mathrm{~d} W_{s}=\frac{Y_{T}-Y_{0}-a T}{T} \stackrel{\mathcal{D}}{\longrightarrow} \mathcal{Y}_{1}-a, \quad \text { as } T \rightarrow \infty .
$$


By Itô's formula and using $b=0$,

$$
\mathrm{d}\left(Y_{t}^{2}\right)=2 Y_{t} \mathrm{~d} Y_{t}+\sigma_{1}^{2} Y_{t} \mathrm{~d} t=2 Y_{t}\left(a \mathrm{~d} t+\sigma_{1} Y_{t}^{\frac{1}{2}} \mathrm{~d} W_{t}\right)+\sigma_{1}^{2} Y_{t} \mathrm{~d} t=\left(2 a+\sigma_{1}^{2}\right) Y_{t} \mathrm{~d} t+2 \sigma_{1} Y_{t}^{\frac{3}{2}} \mathrm{~d} W_{t}
$$

hence

$$
Y_{T}^{2}=Y_{0}^{2}+\left(2 a+\sigma_{1}^{2}\right) \int_{0}^{T} Y_{s} \mathrm{~d} s+2 \sigma_{1} \int_{0}^{T} Y_{s}^{\frac{3}{2}} \mathrm{~d} W_{s}
$$

Consequently,

$$
-\frac{\sigma_{1}}{T^{2}} \int_{0}^{T} Y_{s}^{\frac{3}{2}} \mathrm{~d} W_{s}=-\frac{Y_{T}^{2}-Y_{0}^{2}-\left(2 a+\sigma_{1}^{2}\right) \int_{0}^{T} Y_{s} \mathrm{~d} s}{2 T^{2}} \stackrel{\mathcal{D}}{\longrightarrow}-\frac{\mathcal{Y}_{1}^{2}-\left(2 a+\sigma_{1}^{2}\right) \int_{0}^{1} \mathcal{Y}_{s} \mathrm{~d} s}{2}
$$

as $T \rightarrow \infty$. In a similar way, applying the second equation of (1.1) and using $\beta=0$ and $\gamma=0$, we obtain

$$
\frac{\sigma_{2}}{T} \int_{0}^{T} Y_{s}^{\frac{1}{2}} \mathrm{~d} \widetilde{W}_{s}+\frac{\sigma_{3}}{T} L_{T}=\frac{X_{T}-X_{0}-\alpha T}{T} \stackrel{\mathcal{D}}{\longrightarrow} \mathcal{X}_{1}-\alpha, \quad \text { as } T \rightarrow \infty .
$$

By Itô's formula and using $\beta=0$ and $\gamma=0$,

$$
\begin{aligned}
\mathrm{d}\left(Y_{t} X_{t}\right) & =Y_{t} \mathrm{~d} X_{t}+X_{t} \mathrm{~d} Y_{t}+\varrho \sigma_{1} \sigma_{2} Y_{t} \mathrm{~d} t=Y_{t}\left(\alpha \mathrm{d} t+\sigma_{2} Y_{t}^{\frac{1}{2}} \mathrm{~d} \widetilde{W}_{t}+\sigma_{3} \mathrm{~d} L_{t}\right)+X_{t} \mathrm{~d} Y_{t}+\varrho \sigma_{1} \sigma_{2} Y_{t} \mathrm{~d} t \\
& =\left(\alpha+\varrho \sigma_{1} \sigma_{2}\right) Y_{t} \mathrm{~d} t+\sigma_{2} Y_{t}^{\frac{3}{2}} \mathrm{~d} \widetilde{W}_{t}+X_{t} \mathrm{~d} Y_{t}+\sigma_{3} Y_{t} \mathrm{~d} L_{t},
\end{aligned}
$$

hence

$$
Y_{T} X_{T}=Y_{0} X_{0}+\left(\alpha+\varrho \sigma_{1} \sigma_{2}\right) \int_{0}^{T} Y_{s} \mathrm{~d} s+\sigma_{2} \int_{0}^{T} Y_{s}^{\frac{3}{2}} \mathrm{~d} \widetilde{W}_{s}+\int_{0}^{T} X_{s} \mathrm{~d} Y_{s}+\sigma_{3} \int_{0}^{T} Y_{s} \mathrm{~d} L_{s} .
$$

Consequently,

$$
\begin{aligned}
-\frac{\sigma_{2}}{T^{2}} \int_{0}^{T} Y_{s}^{\frac{3}{2}} \mathrm{~d} \widetilde{W}_{s}-\frac{\sigma_{3}}{T^{2}} \int_{0}^{T} Y_{s} \mathrm{~d} L_{s} & =-\frac{Y_{T} X_{T}-Y_{0} X_{0}-\left(\alpha+\varrho \sigma_{1} \sigma_{2}\right) \int_{0}^{T} Y_{s} \mathrm{~d} s-\int_{0}^{T} X_{s} \mathrm{~d} Y_{s}}{T^{2}} \\
& \stackrel{\mathcal{D}}{\longrightarrow}-\mathcal{Y}_{1} \mathcal{X}_{1}+\left(\alpha+\varrho \sigma_{1} \sigma_{2}\right) \int_{0}^{1} \mathcal{Y}_{s} \mathrm{~d} s+\int_{0}^{1} \mathcal{X}_{s} \mathrm{~d} \mathcal{Y}_{s}
\end{aligned}
$$

as $T \rightarrow \infty$. Again by Itô's formula and using $\beta=0$ and $\gamma=0$,

$$
\mathrm{d}\left(X_{t}^{2}\right)=2 X_{t} \mathrm{~d} X_{t}+\left(\sigma_{2}^{2} Y_{t}+\sigma_{3}^{2}\right) \mathrm{d} t=2 X_{t}\left(\alpha \mathrm{d} t+\sigma_{2} Y_{t}^{\frac{1}{2}} \mathrm{~d} \widetilde{W}_{t}+\sigma_{3} \mathrm{~d} L_{t}\right)+\left(\sigma_{2}^{2} Y_{t}+\sigma_{3}^{2}\right) \mathrm{d} t
$$

hence

$$
X_{T}^{2}=X_{0}^{2}+\int_{0}^{T}\left(2 \alpha X_{s}+\sigma_{2}^{2} Y_{s}+\sigma_{3}^{2}\right) \mathrm{d} s+2 \sigma_{2} \int_{0}^{T} Y_{s}^{\frac{1}{2}} X_{s} \mathrm{~d} \widetilde{W}_{s}+2 \sigma_{3} \int_{0}^{T} X_{s} \mathrm{~d} L_{s} .
$$

Consequently,

$$
\begin{aligned}
-\frac{\sigma_{2}}{T^{2}} \int_{0}^{T} Y_{s}^{\frac{1}{2}} X_{s} \mathrm{~d} \widetilde{W}_{s}-\frac{\sigma_{3}}{T^{2}} \int_{0}^{T} X_{s} \mathrm{~d} L_{s} & =-\frac{X_{T}^{2}-X_{0}^{2}-\int_{0}^{T}\left(2 \alpha X_{s}+\sigma_{2}^{2} Y_{s}+\sigma_{3}^{2}\right) \mathrm{d} s}{2 T^{2}} \\
& \stackrel{\mathcal{D}}{\longrightarrow}-\frac{\mathcal{X}_{1}^{2}-\int_{0}^{1}\left(2 \alpha \mathcal{X}_{s}+\sigma_{2}^{2} \mathcal{Y}_{s}+\sigma_{3}^{2}\right) \mathrm{d} s}{2}
\end{aligned}
$$

as $T \rightarrow \infty$, and we conclude (6.1). 


\section{Asymptotic behavior of CLSE: supercritical case}

First we present an auxiliary lemma about the asymptotic behavior of $\mathbb{E}\left(X_{t}^{2}\right)$ as $t \rightarrow \infty$.

7.1 Lemma. Let us consider the two-factor affine diffusion model (1.1) with $a \in \mathbb{R}_{+}, \quad b \in \mathbb{R}_{--}$, $\alpha, \beta \in \mathbb{R}, \quad \gamma \in(-\infty, b), \sigma_{1} \in \mathbb{R}_{++}, \sigma_{2}, \sigma_{3} \in \mathbb{R}_{+}$and $\varrho \in[-1,1]$ with a random initial value $\left(\eta_{0}, \zeta_{0}\right)$ independent of $\left(W_{t}, B_{t}, L_{t}\right)_{t \in \mathbb{R}_{+}}$satisfying $\mathbb{P}\left(\eta_{0} \in \mathbb{R}_{+}\right)=1$. Then $\sup _{t \in \mathbb{R}_{+}} \mathrm{e}^{2 \gamma t} \mathbb{E}\left(X_{t}^{2}\right)<\infty$.

Proof. By Proposition B.1,

$$
\sup _{t \in \mathbb{R}_{+}} e^{b t} \mathbb{E}\left(Y_{t}\right)=\sup _{t \in \mathbb{R}_{+}}\left(\mathbb{E}\left(Y_{0}\right)+a \int_{0}^{t} \mathrm{e}^{b u} \mathrm{~d} u\right)=\mathbb{E}\left(Y_{0}\right)+a \int_{0}^{\infty} \mathrm{e}^{b u} \mathrm{~d} u<\infty,
$$

since $b<0$. Moreover,

$$
\begin{aligned}
\sup _{t \in \mathbb{R}_{+}} \mathrm{e}^{\gamma t}\left|\mathbb{E}\left(X_{t}\right)\right| & =\sup _{t \in \mathbb{R}_{+}}\left|\mathbb{E}\left(X_{0}\right)+\alpha \int_{0}^{t} \mathrm{e}^{\gamma u} \mathrm{~d} u-\beta \int_{0}^{t} \mathrm{e}^{\gamma u} \mathbb{E}\left(Y_{u}\right) \mathrm{d} u\right| \\
& \leqslant\left|\mathbb{E}\left(X_{0}\right)\right|+|\alpha| \int_{0}^{\infty} \mathrm{e}^{\gamma u} \mathrm{~d} u+|\beta|\left(\sup _{u \in \mathbb{R}_{+}} \mathrm{e}^{b u} \mathbb{E}\left(Y_{u}\right)\right) \int_{0}^{\infty} \mathrm{e}^{(\gamma-b) u} \mathrm{~d} u<\infty,
\end{aligned}
$$

using $\gamma<0$ and $\gamma-b<0$. Again by Proposition B.1,

$$
\begin{aligned}
\sup _{t \in \mathbb{R}_{+}} \mathrm{e}^{2 b t} \mathbb{E}\left(Y_{t}^{2}\right) & =\sup _{t \in \mathbb{R}_{+}}\left(\mathbb{E}\left(Y_{0}^{2}\right)+\left(2 a+\sigma_{1}^{2}\right) \int_{0}^{t} \mathrm{e}^{2 b u} \mathbb{E}\left(Y_{u}\right) \mathrm{d} u\right) \\
& \leqslant \mathbb{E}\left(Y_{0}^{2}\right)+\left(2 a+\sigma_{1}^{2}\right)\left(\sup _{u \in \mathbb{R}_{+}} \mathrm{e}^{b u} \mathbb{E}\left(Y_{u}\right)\right) \int_{0}^{\infty} \mathrm{e}^{b u} \mathrm{~d} u<\infty,
\end{aligned}
$$

using $b<0$. Hence

$$
\begin{aligned}
& \sup _{t \in \mathbb{R}_{+}} \mathrm{e}^{(b+\gamma) t}\left|\mathbb{E}\left(Y_{t} X_{t}\right)\right|=\sup _{t \in \mathbb{R}_{+}} \mid \mathbb{E}\left(Y_{0} X_{0}\right)+a \int_{0}^{t} \mathrm{e}^{(b+\gamma) u} \mathbb{E}\left(X_{u}\right) \mathrm{d} u \\
& \quad+\left(\alpha+\varrho \sigma_{1} \sigma_{2}\right) \int_{0}^{t} \mathrm{e}^{(b+\gamma) u} \mathbb{E}\left(Y_{u}\right) \mathrm{d} u-\beta \int_{0}^{t} \mathrm{e}^{(b+\gamma) u} \mathbb{E}\left(Y_{u}^{2}\right) \mathrm{d} u \mid \\
& \leqslant\left|\mathbb{E}\left(Y_{0} X_{0}\right)\right|+a\left(\sup _{u \in \mathbb{R}_{+}} \mathrm{e}^{\gamma u}\left|\mathbb{E}\left(X_{u}\right)\right|\right) \int_{0}^{\infty} \mathrm{e}^{b u} \mathrm{~d} u+\left(|\alpha|+|\varrho| \sigma_{1} \sigma_{2}\right)\left(\sup _{u \in \mathbb{R}_{+}} \mathrm{e}^{b u} \mathbb{E}\left(Y_{u}\right)\right) \int_{0}^{\infty} \mathrm{e}^{\gamma u} \mathrm{~d} u \\
& +|\beta|\left(\sup _{u \in \mathbb{R}_{+}} \mathrm{e}^{2 b u} \mathbb{E}\left(Y_{u}^{2}\right)\right) \int_{0}^{\infty} \mathrm{e}^{(\gamma-b) u} \mathrm{~d} u<\infty,
\end{aligned}
$$


using $b<0, \gamma<0$ and $\gamma-b<0$. Consequently,

$$
\begin{aligned}
& \sup _{t \in \mathbb{R}_{+}} \mathrm{e}^{2 \gamma t} \mathbb{E}\left(X_{t}^{2}\right)=\sup _{t \in \mathbb{R}_{+}}\left(\mathbb{E}\left(X_{0}^{2}\right)+\alpha \int_{0}^{t} \mathrm{e}^{2 \gamma u} X_{u} \mathrm{~d} u-2 \beta \int_{0}^{t} \mathrm{e}^{2 \gamma u} Y_{u} X_{u} \mathrm{~d} u\right. \\
& \left.+\sigma_{2}^{2} \int_{0}^{t} \mathrm{e}^{2 \gamma u} Y_{u} \mathrm{~d} u+\sigma_{3}^{2} \int_{0}^{t} \mathrm{e}^{2 \gamma u} \mathrm{~d} u\right) \\
& \leqslant \mathbb{E}\left(X_{0}^{2}\right)+|\alpha|\left(\sup _{u \in \mathbb{R}_{+}} \mathrm{e}^{\gamma u}\left|\mathbb{E}\left(X_{u}\right)\right|\right) \int_{0}^{\infty} \mathrm{e}^{\gamma u} \mathrm{~d} u+2|\beta|\left(\sup _{u \in \mathbb{R}_{+}} \mathrm{e}^{(b+\gamma) u}\left|\mathbb{E}\left(Y_{u} X_{u}\right)\right|\right) \int_{0}^{\infty} \mathrm{e}^{(\gamma-b) u} \mathrm{~d} u \\
& +\sigma_{2}^{2}\left(\sup _{u \in \mathbb{R}_{+}} \mathrm{e}^{b u} \mathbb{E}\left(Y_{u}\right)\right) \int_{0}^{\infty} \mathrm{e}^{(2 \gamma-b) u} \mathrm{~d} u+\sigma_{3}^{2} \int_{0}^{\infty} \mathrm{e}^{2 \gamma u} \mathrm{~d} u<\infty
\end{aligned}
$$

using $\gamma<0, \gamma-b<0$ and $2 \gamma-b<0$.

Next we present an auxiliary lemma about the asymptotic behavior of $X_{t}$ as $t \rightarrow \infty$.

7.2 Lemma. Let us consider the two-factor affine diffusion model (1.1) with $a \in \mathbb{R}_{+}, \quad b \in \mathbb{R}_{--}$, $\alpha, \beta \in \mathbb{R}, \quad \gamma \in(-\infty, b), \sigma_{1} \in \mathbb{R}_{++}, \sigma_{2}, \sigma_{3} \in \mathbb{R}_{+}$and $\varrho \in[-1,1]$ with a random initial value $\left(\eta_{0}, \zeta_{0}\right)$ independent of $\left(W_{t}, B_{t}, L_{t}\right)_{t \in \mathbb{R}_{+}}$satisfying $\mathbb{P}\left(\eta_{0} \in \mathbb{R}_{+}\right)=1$. Suppose that $\alpha \beta \in \mathbb{R}_{-}$. Then there exists a random variable $V_{X}$ such that

$$
\mathrm{e}^{\gamma t} X_{t} \stackrel{\text { a.s. }}{\longrightarrow} V_{X} \quad \text { as } t \rightarrow \infty
$$

and, for each $k, \ell \in \mathbb{Z}_{+}$with $k+\ell>0$,

$$
\mathrm{e}^{(k b+\ell \gamma) t} \int_{0}^{t} Y_{u}^{k} X_{u}^{\ell} \mathrm{d} u \stackrel{\text { a.s. }}{\longrightarrow}-\frac{V_{Y}^{k} V_{X}^{\ell}}{k b+\ell \gamma} \quad \text { as } t \rightarrow \infty
$$

where $V_{Y}$ is given in (4.6). If, in addition, $\sigma_{3} \in \mathbb{R}_{++}$or $\left(a-\frac{\sigma_{1}^{2}}{2}\right)\left(1-\varrho^{2}\right) \sigma_{2}^{2} \in \mathbb{R}_{++}$, then the distribution of the random variable $V_{X}$ is absolutely continuous. Particularly, $\mathbb{P}\left(V_{X} \neq 0\right)=1$.

Proof. By (2.2),

$$
\mathbb{E}\left(X_{t} \mid \mathcal{F}_{s}\right)=\mathbb{E}\left(X_{t} \mid Y_{s}, X_{s}\right)=\mathrm{e}^{-\gamma(t-s)} X_{s}+\int_{s}^{t} \mathrm{e}^{-\gamma(t-u)}\left(\alpha-\beta Y_{u}\right) \mathrm{d} u
$$

for all $s, t \in \mathbb{R}_{+}$with $0 \leqslant s \leqslant t$. If $\alpha \in \mathbb{R}_{+}$and $\beta \in \mathbb{R}_{-}$, then

$$
\mathbb{E}\left(\mathrm{e}^{\gamma t} X_{t} \mid \mathcal{F}_{s}^{Y, X}\right)=\mathrm{e}^{\gamma s} X_{s}+\int_{s}^{t} \mathrm{e}^{\gamma u}\left(\alpha-\beta Y_{u}\right) \mathrm{d} u \geqslant \mathrm{e}^{\gamma s} X_{s}
$$

for all $s, t \in \mathbb{R}_{+}$with $0 \leqslant s \leqslant t$, consequently, the process $\left(\mathrm{e}^{\gamma t} X_{t}\right)_{t \in \mathbb{R}_{+}}$is a submartingale with respect to the filtration $\left(\mathcal{F}_{t}^{Y, X}\right)_{t \in \mathbb{R}_{+}}$. If $\alpha \in \mathbb{R}_{-}$and $\beta \in \mathbb{R}_{+}$, then

$$
\mathbb{E}\left(\mathrm{e}^{\gamma t} X_{t} \mid \mathcal{F}_{s}^{Y, X}\right)=\mathrm{e}^{\gamma s} X_{s}+\int_{s}^{t} \mathrm{e}^{\gamma u}\left(\alpha-\beta Y_{u}\right) \mathrm{d} u \leqslant \mathrm{e}^{\gamma s} X_{s}
$$

for all $s, t \in \mathbb{R}_{+}$with $0 \leqslant s \leqslant t$, consequently, the process $\left(\mathrm{e}^{\gamma t} X_{t}\right)_{t \in \mathbb{R}_{+}}$is a supermartingale with respect to the filtration $\left(\mathcal{F}_{t}^{Y, X}\right)_{t \in \mathbb{R}_{+}}$, hence the process $\left(-\mathrm{e}^{\gamma t} X_{t}\right)_{t \in \mathbb{R}_{+}}$is a submartingale with respect 
to the filtration $\left(\mathcal{F}_{t}^{Y, X}\right)_{t \in \mathbb{R}_{+}}$. In both cases, $\sup _{t \in \mathbb{R}_{+}} \mathbb{E}\left(\left|\mathrm{e}^{\gamma t} X_{t}\right|^{2}\right)<\infty$, see Lemma 7.1. Hence, by the submartingale convergence theorem, there exists a random variable $V_{X}$ such that (7.1) holds.

If $\omega \in \Omega$ such that $\mathbb{R}_{+} \ni t \mapsto\left(Y_{t}(\omega), X_{t}(\omega)\right)$ is continuous and $\left(\mathrm{e}^{b t} Y_{t}(\omega), \mathrm{e}^{\gamma t} X_{t}(\omega)\right) \rightarrow$ $\left(V_{Y}(\omega), V_{X}(\omega)\right)$ as $t \rightarrow \infty$, then, by the integral Kronecker Lemma 4.3 with $f(t)=$ $\mathrm{e}^{(k b+\ell \gamma) t} Y_{t}(\omega)^{k} X_{t}(\omega)^{\ell}$ and $a(t)=\mathrm{e}^{-(k b+\ell \gamma) t}, t \in \mathbb{R}_{+}$, we have

$$
\frac{1}{\int_{0}^{t} \mathrm{e}^{-(k b+\ell \gamma) u} \mathrm{~d} u} \int_{0}^{t} \mathrm{e}^{-(k b+\ell \gamma) u}\left(\mathrm{e}^{(k b+\ell \gamma) u} Y_{u}(\omega)^{k} X_{u}(\omega)^{\ell}\right) \mathrm{d} u \rightarrow V_{Y}(\omega)^{k} V_{X}(\omega)^{\ell} \quad \text { as } t \rightarrow \infty .
$$

Here $\int_{0}^{t} \mathrm{e}^{-(k b+\ell \gamma) u} \mathrm{~d} u=-\frac{\mathrm{e}^{-(k b+\ell \gamma) t}-1}{k b+\ell \gamma}, t \in \mathbb{R}_{+}$, thus we conclude (17.2).

Now suppose that $\sigma_{3} \in \mathbb{R}_{++}$or $\left(a-\frac{\sigma_{1}^{2}}{2}\right)\left(1-\varrho^{2}\right) \sigma_{2}^{2} \in \mathbb{R}_{++}$. We are going to show that the random variable $V_{X}$ is absolutely continuous. Put $Z_{t}:=X_{t}-r Y_{t}, t \in \mathbb{R}_{+}$with $r:=\frac{\sigma_{2} \varrho}{\sigma_{1}}$. Then the process $\left(Y_{t}, Z_{t}\right)_{t \in \mathbb{R}_{+}}$is an affine process satisfying

$$
\left\{\begin{array}{l}
\mathrm{d} Y_{t}=\left(a-b Y_{t}\right) \mathrm{d} t+\sigma_{1} \sqrt{Y_{t}} \mathrm{~d} W_{t}, \\
\mathrm{~d} Z_{t}=\left(A-B Y_{t}-\gamma Z_{t}\right) \mathrm{d} t+\Sigma_{2} \sqrt{Y_{t}} \mathrm{~d} B_{t}+\sigma_{3} \mathrm{~d} L_{t} .
\end{array} \quad t \in \mathbb{R}_{+},\right.
$$

where $A:=\alpha-r a, B:=\beta-r(b-\gamma)$ and $\Sigma_{2}:=\sigma_{2} \sqrt{1-\varrho^{2}}$, see Bolyog and Pap [11, Proposition 2.5]. We have

$$
\mathrm{e}^{\gamma t} X_{t}=r \mathrm{e}^{\gamma t} Y_{t}+\mathrm{e}^{\gamma t} Z_{t}=r \mathrm{e}^{\gamma t} Y_{t}+Z_{0}+\int_{0}^{t} \mathrm{e}^{\gamma u}\left(A-B Y_{u}\right) \mathrm{d} u+\Sigma_{2} \int_{0}^{t} \mathrm{e}^{\gamma u} \sqrt{Y_{u}} \mathrm{~d} B_{u}+\sigma_{3} \int_{0}^{t} \mathrm{e}^{\gamma u} \mathrm{~d} L_{u}
$$

where we used (2.2) with $s=0$ multiplied both sides by $\mathrm{e}^{\gamma t}$. Thus the conditional distribution of $\mathrm{e}^{\gamma t} X_{t}$ given $\left(Y_{u}\right)_{u \in[0, t]}$ and $X_{0}$ is a normal distribution with mean $r \mathrm{e}^{\gamma t} Y_{t}+Z_{0}+\int_{0}^{t} \mathrm{e}^{\gamma u}\left(A-B Y_{u}\right) \mathrm{d} u$ and with variance $\Sigma_{2}^{2} \int_{0}^{t} \mathrm{e}^{2 \gamma u} Y_{u} \mathrm{~d} u+\sigma_{3}^{2} \int_{0}^{t} \mathrm{e}^{2 \gamma u} \mathrm{~d} u$. Hence

$$
\begin{aligned}
& \mathbb{E}\left(\mathrm{e}^{\mathrm{i} \lambda \mathrm{e}^{\gamma t} X_{t}} \mid\left(Y_{u}\right)_{u \in[0, t]}, X_{0}\right) \\
& =\exp \left\{\mathrm{i} \lambda\left(r \mathrm{e}^{\gamma t} Y_{t}+Z_{0}+\int_{0}^{t} \mathrm{e}^{\gamma u}\left(A-B Y_{u}\right) \mathrm{d} u\right)-\frac{\lambda^{2}}{2}\left(\Sigma_{2}^{2} \int_{0}^{t} \mathrm{e}^{2 \gamma u} Y_{u} \mathrm{~d} u+\sigma_{3}^{2} \int_{0}^{t} \mathrm{e}^{2 \gamma u} \mathrm{~d} u\right)\right\} .
\end{aligned}
$$

Consequently,

$$
\begin{aligned}
& \left|\mathbb{E}\left(\mathrm{e}^{\mathrm{i} \lambda \mathrm{e}^{\gamma t} X_{t}}\right)\right|=\left|\mathbb{E}\left(\mathbb{E}\left(\mathrm{e}^{\mathrm{i} \lambda \mathrm{e}^{\gamma t} X_{t}} \mid\left(Y_{u}\right)_{u \in[0, t]}, X_{0}\right)\right)\right| \\
& =\left|\mathbb{E}\left(\exp \left\{\mathrm{i} \lambda\left(r \mathrm{e}^{\gamma t} Y_{t}+Z_{0}+\int_{0}^{t} \mathrm{e}^{\gamma u}\left(A-B Y_{u}\right) \mathrm{d} u\right)-\frac{\lambda^{2}}{2}\left(\Sigma_{2}^{2} \int_{0}^{t} \mathrm{e}^{2 \gamma u} Y_{u} \mathrm{~d} u+\sigma_{3}^{2} \int_{0}^{t} \mathrm{e}^{2 \gamma u} \mathrm{~d} u\right)\right\}\right)\right| \\
& \leqslant \mathbb{E}\left(\left|\exp \left\{\mathrm{i} \lambda\left(r \mathrm{e}^{\gamma t} Y_{t}+Z_{0}+\int_{0}^{t} \mathrm{e}^{\gamma u}\left(A-B Y_{u}\right) \mathrm{d} u\right)-\frac{\lambda^{2}}{2}\left(\Sigma_{2}^{2} \int_{0}^{t} \mathrm{e}^{2 \gamma u} Y_{u} \mathrm{~d} u+\sigma_{3}^{2} \int_{0}^{t} \mathrm{e}^{2 \gamma u} \mathrm{~d} u\right)\right\}\right|\right) \\
& =\mathbb{E}\left(\exp \left\{-\frac{\lambda^{2}}{2}\left(\Sigma_{2}^{2} \int_{0}^{t} \mathrm{e}^{2 \gamma u} Y_{u} \mathrm{~d} u+\sigma_{3}^{2} \int_{0}^{t} \mathrm{e}^{2 \gamma u} \mathrm{~d} u\right)\right\}\right) .
\end{aligned}
$$

Convergence (7.1) implies $\mathrm{e}^{\gamma t} X_{t} \stackrel{\mathcal{D}}{\longrightarrow} V_{X}$ as $t \rightarrow \infty$, hence, by the continuity theorem and by the monotone convergence theorem,

$$
\begin{aligned}
\left|\mathbb{E}\left(\mathrm{e}^{\mathrm{i} \lambda V_{X}}\right)\right|=\lim _{t \rightarrow \infty}\left|\mathbb{E}\left(\mathrm{e}^{\mathrm{i} \lambda \mathrm{e}^{\gamma t} X_{t}}\right)\right| & \leqslant \lim _{t \rightarrow \infty} \mathbb{E}\left(\exp \left\{-\frac{\lambda^{2}}{2}\left(\Sigma_{2}^{2} \int_{0}^{t} \mathrm{e}^{2 \gamma u} Y_{u} \mathrm{~d} u+\sigma_{3}^{2} \int_{0}^{t} \mathrm{e}^{2 \gamma u} \mathrm{~d} u\right)\right\}\right) \\
& =\mathbb{E}\left(\exp \left\{-\frac{\lambda^{2}}{2}\left(\Sigma_{2}^{2} \int_{0}^{\infty} \mathrm{e}^{2 \gamma u} Y_{u} \mathrm{~d} u+\sigma_{3}^{2} \int_{0}^{\infty} \mathrm{e}^{2 \gamma u} \mathrm{~d} u\right)\right\}\right) .
\end{aligned}
$$


for all $\lambda \in \mathbb{R}$. If $\sigma_{3} \in \mathbb{R}_{++}$, then we have

$$
\left|\mathbb{E}\left(\mathrm{e}^{\mathrm{i} \lambda V_{X}}\right)\right| \leqslant \exp \left\{-\frac{\sigma_{3}^{2}}{4(-\gamma)} \lambda^{2}\right\}
$$

for all $\lambda \in \mathbb{R}$, hence $\int_{-\infty}^{\infty}\left|\mathbb{E}\left(\mathrm{e}^{\mathrm{i} \lambda V_{X}}\right)\right| \mathrm{d} \lambda<\infty$, implying absolute continuity of the distribution of $V_{X}$.

If $\left(a-\frac{\sigma_{1}^{2}}{2}\right)\left(1-\varrho^{2}\right) \sigma_{2}^{2} \in \mathbb{R}_{++}$, then we have

$$
\left|\mathbb{E}\left(\mathrm{e}^{\mathrm{i} \lambda V_{X}}\right)\right| \leqslant \mathbb{E}\left(\exp \left\{-\frac{\Sigma_{2}^{2}}{2} \lambda^{2} \int_{0}^{\infty} \mathrm{e}^{2 \gamma u} Y_{u} \mathrm{~d} u\right\}\right) \leqslant \mathbb{E}\left(\exp \left\{-\frac{\Sigma_{2}^{2} \mathrm{e}^{4 \gamma}}{2} \lambda^{2} \int_{1}^{2} Y_{u} \mathrm{~d} u\right\}\right)
$$

for all $\lambda \in \mathbb{R}$. Applying the comparison theorem (see, e.g., Karatzas and Shreve [24, 5.2.18]), we obtain $\mathbb{P}\left(\mathcal{Y}_{t} \leqslant Y_{t}\right.$ for all $\left.t \in \mathbb{R}_{+}\right)=1$, where $\left(\mathcal{Y}_{t}\right)_{t \in \mathbb{R}_{+}}$is the unique strong solution of the SDE

$$
\mathrm{d} \mathcal{Y}_{t}=\left(a-b \mathcal{Y}_{t}\right) \mathrm{d} t+\sigma_{1} \sqrt{\mathcal{Y}_{t}} \mathrm{~d} W_{t}, \quad t \in[0, \infty),
$$

with initial value $\mathcal{Y}_{0}=0$. Consequently, taking into account $\Sigma_{2}=\sigma_{2} \sqrt{1-\varrho^{2}}>0$, we obtain

$$
\begin{aligned}
& \int_{-\infty}^{\infty}\left|\mathbb{E}\left(\mathrm{e}^{\mathrm{i} \lambda V_{X}}\right)\right| \mathrm{d} \lambda \leqslant \int_{-\infty}^{\infty} \mathbb{E}\left(\exp \left\{-\frac{\Sigma_{2}^{2} \mathrm{e}^{4 \gamma}}{2} \lambda^{2} \int_{1}^{2} \mathcal{Y}_{u} \mathrm{~d} u\right\}\right) \mathrm{d} \lambda \\
& =\mathbb{E}\left(\int_{-\infty}^{\infty} \exp \left\{-\frac{\Sigma_{2}^{2} \mathrm{e}^{4 \gamma}}{2} \lambda^{2} \int_{1}^{2} \mathcal{Y}_{u} \mathrm{~d} u\right\} \mathrm{d} \lambda\right)=\mathbb{E}\left(\frac{\sqrt{2 \pi}}{\Sigma_{2} \mathrm{e}^{2 \gamma} \sqrt{\int_{1}^{2} \mathcal{Y}_{u} \mathrm{~d} u}}\right)=\frac{\sqrt{2 \pi}}{\Sigma_{2} \mathrm{e}^{2 \gamma}} \mathbb{E}\left(\frac{1}{\sqrt{\int_{1}^{2} \mathcal{Y}_{u} \mathrm{~d} u}}\right)<\infty
\end{aligned}
$$

whenever

$$
\mathbb{E}\left(\frac{1}{\sqrt{\int_{1}^{2} \mathcal{Y}_{u} \mathrm{~d} u}}\right)<\infty
$$

By the Cauchy-Schwarz inequality, we have

$$
1=\left(\int_{1}^{2} \sqrt{\mathcal{Y}_{u}} \cdot \frac{1}{\sqrt{\mathcal{Y}_{s}}} \mathrm{~d} u\right)^{2} \leqslant \int_{1}^{2} \mathcal{Y}_{u} \mathrm{~d} u \int_{1}^{2} \frac{1}{\mathcal{Y}_{u}} \mathrm{~d} u
$$

hence

$$
\mathbb{E}\left(\frac{1}{\sqrt{\int_{1}^{2} \mathcal{Y}_{u} \mathrm{~d} u}}\right) \leqslant \mathbb{E}\left(\sqrt{\int_{1}^{2} \frac{1}{\mathcal{Y}_{u}} \mathrm{~d} u}\right) \leqslant \sqrt{\mathbb{E}\left(\int_{1}^{2} \frac{1}{\mathcal{Y}_{u}} \mathrm{~d} u\right)}=\sqrt{\int_{1}^{2} \mathbb{E}\left(\frac{1}{\mathcal{Y}_{u}}\right) \mathrm{d} u}
$$

For each $u \in \mathbb{R}_{++}$, we have $\mathcal{Y}_{u} \stackrel{\mathcal{D}}{=} c(u) \xi$, where the distribution of $\xi$ has a chi-square distribution with degrees of freedom $\frac{4 a}{\sigma_{1}^{2}}$ and $c(u):=\frac{\sigma_{1}^{2}}{4} \int_{0}^{u} \mathrm{e}^{-b v} \mathrm{~d} v=\frac{\sigma_{1}^{2}\left(\mathrm{e}^{-b u}-1\right)}{4(-b)}$, see Proposition B.1. Hence

$$
\mathbb{E}\left(\frac{1}{\mathcal{Y}_{u}}\right)=\frac{1}{c(u)} \mathbb{E}\left(\frac{1}{\xi}\right)
$$

where $\mathbb{E}\left(\frac{1}{\xi}\right)<\infty$, since the density of $\xi$ has the form

$$
\mathbb{R} \ni x \mapsto \frac{1}{2^{\frac{2 a}{\sigma_{1}^{2}}} \Gamma\left(\frac{2 a}{\sigma_{1}^{2}}\right)} x^{\frac{2 a}{\sigma_{1}^{2}}-1} \mathrm{e}^{-\frac{x}{2}} \mathbb{1}_{\mathbb{R}_{++}}(x)
$$


and the assumption $a-\frac{\sigma_{1}^{2}}{2}>0$ yields $\frac{2 a}{\sigma_{1}^{2}}-1>0$. Consequently,

$$
\int_{1}^{2} \mathbb{E}\left(\frac{1}{\mathcal{Y}_{u}}\right) \mathrm{d} u=\mathbb{E}\left(\frac{1}{\xi}\right) \int_{1}^{2} \frac{1}{c(u)} \mathrm{d} u=\mathbb{E}\left(\frac{1}{\xi}\right) \int_{1}^{2} \frac{4(-b)}{\sigma_{1}^{2}\left(\mathrm{e}^{-b u}-1\right)} \mathrm{d} u<\infty,
$$

thus we obtain (7.3) , and hence $\int_{-\infty}^{\infty}\left|\mathbb{E}\left(\mathrm{e}^{\mathrm{i} \lambda V_{X}}\right)\right| \mathrm{d} \lambda<\infty$, and we conclude absolute continuity of the distribution of $V_{X}$.

7.3 Theorem. Let us consider the two-factor affine diffusion model (1.1) with $a \in \mathbb{R}_{+}, \quad b \in \mathbb{R}_{--}$, $\alpha, \beta \in \mathbb{R}, \gamma \in(-\infty, b), \sigma_{1} \in \mathbb{R}_{++}, \sigma_{2}, \sigma_{3} \in \mathbb{R}_{+}$and $\varrho \in[-1,1]$ with a random initial value $\left(\eta_{0}, \zeta_{0}\right)$ independent of $\left(W_{t}, B_{t}, L_{t}\right)_{t \in \mathbb{R}_{+}}$satisfying $\mathbb{P}\left(\eta_{0} \in \mathbb{R}_{+}\right)=1$. Suppose that $\alpha \beta \in \mathbb{R}_{-}$. Suppose that $\sigma_{3} \in \mathbb{R}_{++}$or $\left(a-\frac{\sigma_{1}^{2}}{2}\right)\left(1-\varrho^{2}\right) \sigma_{2}^{2} \in \mathbb{R}_{++}$. Then

$$
\left[\begin{array}{c}
T \mathrm{e}^{\frac{b T}{2}}\left(\widehat{a}_{T}-a\right) \\
\mathrm{e}^{-\frac{b T}{2}}\left(\widehat{b}_{T}-b\right) \\
T \mathrm{e}^{\frac{b T}{2}}\left(\widehat{\alpha}_{T}-\alpha\right) \\
\mathrm{e}^{-b T / 2}\left(\widehat{\beta}_{T}-\beta\right) \\
\mathrm{e}^{\frac{(b-2 \gamma) T}{2}}\left(\widehat{\gamma}_{T}-\gamma\right)
\end{array}\right] \stackrel{\mathcal{D}}{\longrightarrow} \boldsymbol{V}^{-1} \boldsymbol{\eta} \boldsymbol{\xi}
$$

as $T \rightarrow \infty$ with

$$
\boldsymbol{V}:=\left[\begin{array}{ccccc}
1 & \frac{V_{Y}}{b} & 0 & 0 & 0 \\
0 & -\frac{V_{Y}^{2}}{2 b} & 0 & 0 & 0 \\
0 & 0 & 1 & \frac{V_{Y}}{b} & \frac{V_{X}}{\gamma} \\
0 & 0 & 0 & -\frac{V_{Y}^{2}}{2 b} & -\frac{V_{Y} V_{X}}{b+\gamma} \\
0 & 0 & 0 & -\frac{V_{Y} V_{X}}{b+\gamma} & -\frac{V_{X}^{2}}{2 \gamma}
\end{array}\right]
$$

where $V_{Y}$ and $V_{X}$ are given in (4.6) and (17.1), respectively, $\boldsymbol{\eta}$ is a $5 \times 5$ random matrix such that

$$
\boldsymbol{\eta} \boldsymbol{\eta}^{\top}=\left[\begin{array}{ccccc}
-\frac{\sigma_{1}^{2} V_{Y}}{b} & \frac{\sigma_{1}^{2} V_{Y}^{2}}{2 b} & -\frac{\varrho \sigma_{1} \sigma_{2} V_{Y}}{b} & \frac{\varrho \sigma_{1} \sigma_{2} V_{Y}^{2}}{2 b} & \frac{\varrho \sigma_{1} \sigma_{2} V_{Y} V_{X}}{b+\gamma} \\
\frac{\sigma_{1}^{2} V_{Y}^{2}}{2 b} & -\frac{\sigma_{1}^{2} V_{Y}^{3}}{3 b} & \frac{\varrho \sigma_{1} \sigma_{2} V_{Y}^{2}}{2 b} & -\frac{\varrho \sigma_{1} \sigma_{2} V_{Y}^{3}}{3 b} & -\frac{\varrho \sigma_{1} \sigma_{2} V_{Y}^{2} V_{X}}{2 b+\gamma} \\
-\frac{\varrho \sigma_{1} \sigma_{2} V_{Y}}{b} & \frac{\varrho \sigma_{1} \sigma_{2} V_{Y}^{2}}{2 b} & -\frac{\sigma_{2}^{2} V_{Y}}{b} & \frac{\sigma_{2}^{2} V_{Y}^{2}}{2 b} & \frac{\sigma_{2}^{2} V_{Y} V_{X}}{b+\gamma} \\
\frac{\varrho \sigma_{1} \sigma_{2} V_{Y}^{2}}{2 b} & -\frac{\varrho \sigma_{1} \sigma_{2} V_{Y}^{3}}{3 b} & \frac{\sigma_{2}^{2} V_{Y}^{2}}{2 b} & -\frac{\sigma_{2}^{2} V_{Y}^{3}}{3 b} & -\frac{\sigma_{2}^{2} V_{Y}^{2} V_{X}}{2 b+\gamma} \\
\frac{\varrho \sigma_{1} \sigma_{2} V_{Y} V_{X}}{b+\gamma} & -\frac{\varrho \sigma_{1} \sigma_{2} V_{Y}^{2} V_{X}}{2 b+\gamma} & \frac{\sigma_{2}^{2} V_{Y} V_{X}}{b+\gamma} & -\frac{\sigma_{2}^{2} V_{Y}^{2} V_{X}}{2 b+\gamma} & -\frac{\sigma_{2}^{2} V_{Y} V_{X}^{2}}{b+2 \gamma}
\end{array}\right],
$$

and $\boldsymbol{\xi}$ is a 5-dimensional standard normally distributed random vector independent of $\left(V_{Y}, V_{X}\right)$.

Proof. We have

$$
\left[\begin{array}{c}
T \mathrm{e}^{\frac{b T}{2}}\left(\widehat{a}_{T}-a\right) \\
\mathrm{e}^{-\frac{b T}{2}}\left(\widehat{b}_{T}-b\right) \\
T \mathrm{e}^{\frac{b T}{2}}\left(\widehat{\alpha}_{T}-\alpha\right) \\
\mathrm{e}^{-\frac{b T}{2}}\left(\widehat{\beta}_{T}-\beta\right) \\
\mathrm{e}^{\frac{(b-2 \gamma) T}{2}}\left(\widehat{\gamma}_{T}-\gamma\right)
\end{array}\right]=\operatorname{diag}\left(T \mathrm{e}^{\frac{b T}{2}}, \mathrm{e}^{-\frac{b T}{2}}, T \mathrm{e}^{\frac{b T}{2}}, \mathrm{e}^{-\frac{b T}{2}}, \mathrm{e}^{\frac{(b-2 \gamma) T}{2}}\right)\left(\widehat{\boldsymbol{\theta}}_{T}-\boldsymbol{\theta}\right)
$$


where, by (3.7),

$$
\widehat{\boldsymbol{\theta}}_{T}-\boldsymbol{\theta}=\boldsymbol{G}_{T}^{-1} \boldsymbol{h}_{T}=\left[\begin{array}{cc}
\boldsymbol{G}_{T}^{(1)} & \mathbf{0} \\
\mathbf{0} & \boldsymbol{G}_{T}^{(2)}
\end{array}\right]^{-1}\left[\begin{array}{l}
\boldsymbol{h}_{T}^{(1)} \\
\boldsymbol{h}_{T}^{(2)}
\end{array}\right] .
$$

We are going to apply Theorem D.2 for the continuous local martingale $\left(\boldsymbol{h}_{T}\right)_{T \in \mathbb{R}_{+}}$with quadratic variation process $\langle\boldsymbol{h}\rangle_{T}=\widetilde{\boldsymbol{G}}_{T}, T \in \mathbb{R}_{+}$(introduced in the proof of Theorem 5.1). With scaling matrices

$$
\boldsymbol{Q}(T):=\operatorname{diag}\left(\mathrm{e}^{\frac{b T}{2}}, \mathrm{e}^{\frac{3 b T}{2}}, \mathrm{e}^{\frac{b T}{2}}, \mathrm{e}^{\frac{3 b T}{2}}, \mathrm{e}^{\frac{(b+2 \gamma) T}{2}}\right), \quad T \in \mathbb{R}_{++},
$$

by (17.2), we have

$$
\boldsymbol{Q}(T)\langle\boldsymbol{h}\rangle_{T} \boldsymbol{Q}(T)^{\top} \stackrel{\text { a.s. }}{\longrightarrow}\left[\begin{array}{ccccc}
-\frac{\sigma_{1}^{2} V_{Y}}{b} & \frac{\sigma_{1}^{2} V_{Y}^{2}}{2 b} & -\frac{\varrho \sigma_{1} \sigma_{2} V_{Y}}{b} & \frac{\varrho \sigma_{1} \sigma_{2} V_{Y}^{2}}{2 b} & \frac{\varrho \sigma_{1} \sigma_{2} V_{Y} V_{X}}{b+\gamma} \\
\frac{\sigma_{1}^{2} V_{Y}^{2}}{2 b} & -\frac{\sigma_{1}^{2} V_{Y}^{3}}{3 b} & \frac{\varrho \sigma_{1} \sigma_{2} V_{Y}^{2}}{2 b} & -\frac{\varrho \sigma_{1} \sigma_{2} V_{Y}^{3}}{3 b} & -\frac{\varrho \sigma_{1} \sigma_{2} V_{Y}^{2} V_{X}}{2 b+\gamma} \\
-\frac{\varrho \sigma_{1} \sigma_{2} V_{Y}}{b} & \frac{\varrho \sigma_{1} \sigma_{2} V_{Y}^{2}}{2 b} & -\frac{\sigma_{2}^{2} V_{Y}}{b} & \frac{\sigma_{2}^{2} V_{Y}^{2}}{2 b} & \frac{\sigma_{2}^{2} V_{Y} V_{X}}{b+\gamma} \\
\frac{\varrho \sigma_{1} \sigma_{2} V_{Y}^{2}}{2 b} & -\frac{\varrho \sigma_{1} \sigma_{2} V_{Y}^{3}}{3 b} & \frac{\sigma_{2}^{2} V_{Y}^{2}}{2 b} & -\frac{\sigma_{2}^{2} V_{Y}^{3}}{3 b} & -\frac{\sigma_{2}^{2} V_{Y}^{2} V_{X}}{2 b+\gamma} \\
\frac{\varrho \sigma_{1} \sigma_{2} V_{Y} V_{X}}{b+\gamma} & -\frac{\varrho \sigma_{1} \sigma_{2} V_{Y}^{2} V_{X}}{2 b+\gamma} & \frac{\sigma_{2}^{2} V_{Y} V_{X}}{b+\gamma} & -\frac{\sigma_{2}^{2} V_{Y}^{2} V_{X}}{2 b+\gamma} & -\frac{\sigma_{2}^{2} V_{Y} V_{X}^{2}}{b+2 \gamma}
\end{array}\right]=\boldsymbol{\eta} \boldsymbol{\eta}^{\top}
$$

as $T \rightarrow \infty$. Hence by Theorem D.2, for each random matrix $\boldsymbol{A}$ defined on $(\Omega, \mathcal{F}, \mathbb{P})$, we obtain

$$
\left(\boldsymbol{Q}(T) \boldsymbol{h}_{T}, \boldsymbol{A}\right) \stackrel{\mathcal{D}}{\longrightarrow}(\boldsymbol{\eta} \boldsymbol{\xi}, \boldsymbol{A}) \quad \text { as } T \rightarrow \infty,
$$

where $\boldsymbol{\xi}$ is a 5-dimensional standard normally distributed random vector independent of $(\boldsymbol{\eta}, \boldsymbol{A})$. The aim of the following discussion is to include appropriate scaling matrices for $\boldsymbol{G}_{T}$. The matrices $\boldsymbol{G}_{T}^{(1)}$ and $\boldsymbol{G}_{T}^{(2)}$ can be written in the form

$$
\boldsymbol{G}_{T}^{(1)}=\operatorname{diag}\left(T^{\frac{1}{2}}, \mathrm{e}^{-b T}\right)\left[\begin{array}{cc}
1 & -\frac{\mathrm{e}^{b T}}{\sqrt{T}} \int_{0}^{T} Y_{s} \mathrm{~d} s \\
-\frac{\mathrm{e}^{b T}}{\sqrt{T}} \int_{0}^{T} Y_{s} \mathrm{~d} s & \mathrm{e}^{2 b T} \int_{0}^{T} Y_{s}^{2} \mathrm{~d} s
\end{array}\right] \operatorname{diag}\left(T^{\frac{1}{2}}, \mathrm{e}^{-b T}\right)
$$

and

$$
\begin{aligned}
\boldsymbol{G}_{T}^{(2)}= & \operatorname{diag}\left(T^{\frac{1}{2}}, \mathrm{e}^{-b T}, \mathrm{e}^{-\gamma T}\right)\left[\begin{array}{ccc}
1 & -\frac{\mathrm{e}^{b T}}{\sqrt{T}} \int_{0}^{T} Y_{s} \mathrm{~d} s & -\frac{\mathrm{e}^{\gamma T}}{\sqrt{T}} \int_{0}^{T} X_{s} \mathrm{~d} s \\
-\frac{\mathrm{e}^{b T}}{\sqrt{T}} \int_{0}^{T} Y_{s} \mathrm{~d} s & \mathrm{e}^{2 b T} \int_{0}^{T} Y_{s}^{2} \mathrm{~d} s & \mathrm{e}^{(b+\gamma) T} \int_{0}^{T} Y_{s} X_{s} \mathrm{~d} s \\
-\frac{\mathrm{e}^{\gamma T}}{\sqrt{T}} \int_{0}^{T} X_{s} \mathrm{~d} s & \mathrm{e}^{(b+\gamma) T} \int_{0}^{T} Y_{s} X_{s} \mathrm{~d} s & \mathrm{e}^{2 \gamma T} \int_{0}^{T} X_{s}^{2} \mathrm{~d} s
\end{array}\right] \\
& \times \operatorname{diag}\left(T^{\frac{1}{2}}, \mathrm{e}^{-b T}, \mathrm{e}^{-\gamma T}\right),
\end{aligned}
$$

hence the matrices $\left(\boldsymbol{G}_{T}^{(1)}\right)^{-1}$ and $\left(\boldsymbol{G}_{T}^{(2)}\right)^{-1}$ can be written in the form

$$
\left(\boldsymbol{G}_{T}^{(1)}\right)^{-1}=\operatorname{diag}\left(T^{-\frac{1}{2}}, \mathrm{e}^{b T}\right)\left[\begin{array}{cc}
1 & -\frac{\mathrm{e}^{b T}}{\sqrt{T}} \int_{0}^{T} Y_{s} \mathrm{~d} s \\
-\frac{\mathrm{e}^{b T}}{\sqrt{T}} \int_{0}^{T} Y_{s} \mathrm{~d} s & \mathrm{e}^{2 b T} \int_{0}^{T} Y_{s}^{2} \mathrm{~d} s
\end{array}\right]^{-1} \operatorname{diag}\left(T^{-\frac{1}{2}}, \mathrm{e}^{b T}\right)
$$

and

$$
\begin{aligned}
\left(\boldsymbol{G}_{T}^{(2)}\right)^{-1}= & \operatorname{diag}\left(T^{-\frac{1}{2}}, \mathrm{e}^{b T}, \mathrm{e}^{\gamma T}\right)\left[\begin{array}{ccc}
1 & -\frac{\mathrm{e}^{b T}}{\sqrt{T}} \int_{0}^{T} Y_{s} \mathrm{~d} s & -\frac{\mathrm{e}^{\gamma T}}{\sqrt{T}} \int_{0}^{T} X_{s} \mathrm{~d} s \\
-\frac{\mathrm{e}^{b T}}{\sqrt{T}} \int_{0}^{T} Y_{s} \mathrm{~d} s & \mathrm{e}^{2 b T} \int_{0}^{T} Y_{s}^{2} \mathrm{~d} s & \mathrm{e}^{(b+\gamma) T} \int_{0}^{T} Y_{s} X_{s} \mathrm{~d} s \\
-\frac{\mathrm{e}^{\gamma T}}{\sqrt{T}} \int_{0}^{T} X_{s} \mathrm{~d} s & \mathrm{e}^{(b+\gamma) T} \int_{0}^{T} Y_{s} X_{s} \mathrm{~d} s & \mathrm{e}^{2 \gamma T} \int_{0}^{T} X_{s}^{2} \mathrm{~d} s
\end{array}\right]^{-1} \\
& \times \operatorname{diag}\left(T^{-\frac{1}{2}}, \mathrm{e}^{b T}, \mathrm{e}^{\gamma T}\right) .
\end{aligned}
$$


We have

$$
\begin{aligned}
& \operatorname{diag}\left(T \mathrm{e}^{\frac{b T}{2}}, \mathrm{e}^{-\frac{b T}{2}}, T \mathrm{e}^{\frac{b T}{2}}, \mathrm{e}^{-\frac{b T}{2}}, \mathrm{e}^{\frac{(b-2 \gamma) T}{2}}\right) \operatorname{diag}\left(T^{-\frac{1}{2}}, \mathrm{e}^{b T}, T^{-\frac{1}{2}}, \mathrm{e}^{b T}, \mathrm{e}^{\gamma T}\right) \\
& =\operatorname{diag}\left(T^{\frac{1}{2}} \mathrm{e}^{\frac{b T}{2}}, \mathrm{e}^{\frac{b T}{2}}, T^{\frac{1}{2}} \mathrm{e}^{\frac{b T}{2}}, \mathrm{e}^{\frac{b T}{2}}, \mathrm{e}^{\frac{b T}{2}}\right)
\end{aligned}
$$

and

$$
\begin{aligned}
& \operatorname{diag}\left(T^{-\frac{1}{2}}, \mathrm{e}^{b T}, T^{-\frac{1}{2}}, \mathrm{e}^{b T}, \mathrm{e}^{\gamma T}\right) \boldsymbol{Q}(T)^{-1} \\
& =\operatorname{diag}\left(T^{-\frac{1}{2}}, \mathrm{e}^{b T}, T^{-\frac{1}{2}}, \mathrm{e}^{b T}, \mathrm{e}^{\gamma T}\right) \operatorname{diag}\left(\mathrm{e}^{-\frac{b T}{2}}, \mathrm{e}^{-\frac{3 b T}{2}}, \mathrm{e}^{-\frac{b T}{2}}, \mathrm{e}^{-\frac{3 b T}{2}}, \mathrm{e}^{-\frac{(b+2 \gamma) T}{2}}\right) \\
& =\operatorname{diag}\left(T^{-\frac{1}{2}} \mathrm{e}^{-\frac{b T}{2}}, \mathrm{e}^{-\frac{b T}{2}}, T^{-\frac{1}{2}} \mathrm{e}^{-\frac{b T}{2}}, \mathrm{e}^{-\frac{b T}{2}}, \mathrm{e}^{-\frac{b T}{2}}\right) .
\end{aligned}
$$

Moreover,

$$
\begin{aligned}
& \operatorname{diag}\left(T^{\frac{1}{2}} \mathrm{e}^{\frac{b T}{2}}, \mathrm{e}^{\frac{b T}{2}}\right)\left[\begin{array}{cc}
1 & -\frac{\mathrm{e}^{b T}}{\sqrt{T}} \int_{0}^{T} Y_{s} \mathrm{~d} s \\
-\frac{\mathrm{e}^{b T}}{\sqrt{T}} \int_{0}^{T} Y_{s} \mathrm{~d} s & \mathrm{e}^{2 b T} \int_{0}^{T} Y_{s}^{2} \mathrm{~d} s
\end{array}\right] \operatorname{diag}\left(T^{-\frac{1}{2}} \mathrm{e}^{-\frac{b T}{2}}, \mathrm{e}^{-\frac{b T}{2}}\right) \\
& =\left[\begin{array}{cc}
1 & -\mathrm{e}^{b T} \int_{0}^{T} Y_{s} \mathrm{~d} s \\
-\frac{\mathrm{e}^{b T}}{T} \int_{0}^{T} Y_{s} \mathrm{~d} s & \mathrm{e}^{2 b T} \int_{0}^{T} Y_{s}^{2} \mathrm{~d} s
\end{array}\right]=: \boldsymbol{J}_{T}^{(1)}
\end{aligned}
$$

and

$$
\begin{aligned}
& \operatorname{diag}\left(T^{\frac{1}{2}} \mathrm{e}^{\frac{b T}{2}}, \mathrm{e}^{\frac{b T}{2}}, \mathrm{e}^{\frac{b T}{2}}\right)\left[\begin{array}{ccc}
1 & -\frac{\mathrm{e}^{b T}}{\sqrt{T}} \int_{0}^{T} Y_{s} \mathrm{~d} s & -\frac{\mathrm{e}^{\gamma T}}{\sqrt{T}} \int_{0}^{T} X_{s} \mathrm{~d} s \\
-\frac{\mathrm{e}^{b T}}{\sqrt{T}} \int_{0}^{T} Y_{s} \mathrm{~d} s & \mathrm{e}^{2 b T} \int_{0}^{T} Y_{s}^{2} \mathrm{~d} s & \mathrm{e}^{(b+\gamma) T} \int_{0}^{T} Y_{s} X_{s} \mathrm{~d} s \\
-\frac{\mathrm{e}^{\gamma T}}{\sqrt{T}} \int_{0}^{T} X_{s} \mathrm{~d} s & \mathrm{e}^{(b+\gamma) T} \int_{0}^{T} Y_{s} X_{s} \mathrm{~d} s & \mathrm{e}^{2 \gamma T} \int_{0}^{T} X_{s}^{2} \mathrm{~d} s
\end{array}\right] \\
& \times \operatorname{diag}\left(T^{-\frac{1}{2}} \mathrm{e}^{-\frac{b T}{2}}, \mathrm{e}^{-\frac{b T}{2}}, \mathrm{e}^{-\frac{b T}{2}}\right) \\
& =\left[\begin{array}{ccc}
1 & -\mathrm{e}^{b T} \int_{0}^{T} Y_{s} \mathrm{~d} s & -\mathrm{e}^{\gamma T} \int_{0}^{T} X_{s} \mathrm{~d} s \\
-\frac{\mathrm{e}^{b T}}{T} \int_{0}^{T} Y_{s} \mathrm{~d} s & \mathrm{e}^{2 b T} \int_{0}^{T} Y_{s}^{2} \mathrm{~d} s & \mathrm{e}^{(b+\gamma) T} \int_{0}^{T} Y_{s} X_{s} \mathrm{~d} s \\
-\frac{\mathrm{e}^{\gamma T}}{T} \int_{0}^{T} X_{s} \mathrm{~d} s & \mathrm{e}^{(b+\gamma) T} \int_{0}^{T} Y_{s} X_{s} \mathrm{~d} s & \mathrm{e}^{2 \gamma T} \int_{0}^{T} X_{s}^{2} \mathrm{~d} s
\end{array}\right]=: \boldsymbol{J}_{T}^{(2)}
\end{aligned}
$$

Consequently,

$$
\left[\begin{array}{c}
T \mathrm{e}^{\frac{b T}{2}}\left(\widehat{a}_{T}-a\right) \\
\mathrm{e}^{-\frac{b T}{2}}\left(\widehat{b}_{T}-b\right) \\
T \mathrm{e}^{\frac{b T}{2}}\left(\widehat{\alpha}_{T}-\alpha\right) \\
\mathrm{e}^{-\frac{b T}{2}}\left(\widehat{\beta}_{T}-\beta\right) \\
\mathrm{e}^{\frac{(b-2 \gamma) T}{2}}\left(\widehat{\gamma}_{T}-\gamma\right)
\end{array}\right]=\operatorname{diag}\left(\boldsymbol{J}_{T}^{(1)}, \boldsymbol{J}_{T}^{(2)}\right)^{-1} \boldsymbol{Q}(T) \boldsymbol{h}_{T}
$$

where, by Lemma 7.2 ,

$$
\operatorname{diag}\left(\boldsymbol{J}_{T}^{(1)}, \boldsymbol{J}_{T}^{(2)}\right) \stackrel{\mathbb{P}}{\longrightarrow} \boldsymbol{V} \quad \text { as } T \rightarrow \infty .
$$

By (7.5) with $\boldsymbol{A}=\boldsymbol{V}$, by (7.6) and by Theorem 2.7 (iv) of van der Vaart [28, we obtain

$$
\left(\boldsymbol{Q}(T) \boldsymbol{h}_{T}, \operatorname{diag}\left(\boldsymbol{J}_{T}^{(1)}, \boldsymbol{J}_{T}^{(2)}\right)\right) \stackrel{\mathcal{D}}{\longrightarrow}(\boldsymbol{\eta} \boldsymbol{\xi}, \boldsymbol{V}) \quad \text { as } T \rightarrow \infty .
$$


The random matrix $\boldsymbol{V}$ is invertible almost surely, since

$$
\operatorname{det}(\boldsymbol{V})=-\frac{(b-\gamma)^{2} V_{Y}^{4} V_{X}^{2}}{8(b+\gamma)^{2} b^{2} \gamma}>0
$$

almost surely by Lemma 7.2. Consequently, $\operatorname{diag}\left(\boldsymbol{J}_{T}^{(1)}, \boldsymbol{J}_{T}^{(2)}\right)^{-1} \boldsymbol{Q}(T) \boldsymbol{h}_{T} \stackrel{\mathcal{D}}{\longrightarrow} \boldsymbol{V}^{-1} \boldsymbol{\eta} \boldsymbol{\xi}$ as $T \rightarrow \infty$.

\section{Appendix}

\section{A Stationarity and exponential ergodicity}

The following result states the existence of a unique stationary distribution of the affine diffusion process given by the SDE (1.1), see Bolyog and Pap [11, Theorem 3.1]. Let $\mathbb{C}_{-}:=\{z \in \mathbb{C}: \operatorname{Re}(z) \leqslant 0\}$.

A.1 Theorem. Let us consider the two-factor affine diffusion model (1.1) with $a \in \mathbb{R}_{+}, \quad b \in \mathbb{R}_{++}$, $\alpha, \beta \in \mathbb{R}, \quad \gamma \in \mathbb{R}_{++}, \quad \sigma_{1}, \sigma_{2}, \sigma_{3} \in \mathbb{R}_{+}, \varrho \in[-1,1]$, and with a random initial value $\left(\eta_{0}, \zeta_{0}\right)$ independent of $\left(W_{t}, B_{t}, L_{t}\right)_{t \in \mathbb{R}_{+}}$satisfying $\mathbb{P}\left(\eta_{0} \in \mathbb{R}_{+}\right)=1$. Then

(i) $\left(Y_{t}, X_{t}\right) \stackrel{\mathcal{D}}{\longrightarrow}\left(Y_{\infty}, X_{\infty}\right)$ as $t \rightarrow \infty$, and we have

$$
\mathbb{E}\left(\mathrm{e}^{u_{1} Y_{\infty}+\mathrm{i} \lambda_{2} X_{\infty}}\right)=\exp \left\{a \int_{0}^{\infty} \kappa_{s}\left(u_{1}, \lambda_{2}\right) \mathrm{d} s+\mathrm{i} \frac{\alpha}{\gamma} \lambda_{2}-\frac{\sigma_{3}^{2}}{4 \gamma} \lambda_{2}^{2}\right\}
$$

for $\left(u_{1}, \lambda_{2}\right) \in \mathbb{C}_{-} \times \mathbb{R}$, where $\kappa_{t}\left(u_{1}, \lambda_{2}\right), t \in \mathbb{R}_{+}$, is the unique solution of the (deterministic) differential equation

$$
\left\{\begin{aligned}
\frac{\partial \kappa_{t}}{\partial t}\left(u_{1}, \lambda_{2}\right)= & -b \kappa_{t}\left(u_{1}, \lambda_{2}\right)-\mathrm{i} \beta \mathrm{e}^{-\gamma t} \lambda_{2}+\frac{1}{2} \sigma_{1}^{2} \kappa_{t}\left(u_{1}, \lambda_{2}\right)^{2} \\
& +\mathrm{i} \varrho \sigma_{1} \sigma_{2} \mathrm{e}^{-\gamma t} \lambda_{2} \kappa_{t}\left(u_{1}, \lambda_{2}\right)-\frac{1}{2} \sigma_{2}^{2} \mathrm{e}^{-2 \gamma t} \lambda_{2}^{2} \\
\kappa_{0}\left(u_{1}, \lambda_{2}\right)= & u_{1}
\end{aligned}\right.
$$

(ii) supposing that the random initial value $\left(\eta_{0}, \zeta_{0}\right)$ has the same distribution as $\left(Y_{\infty}, X_{\infty}\right)$ given in part $(\mathrm{i}),\left(Y_{t}, X_{t}\right)_{t \in \mathbb{R}_{+}}$is strictly stationary.

In the subcritical case, the following result states the exponential ergodicity and a strong law of large numbers for the process $\left(Y_{t}, X_{t}\right)_{t \in \mathbb{R}_{+}}$, see Bolyog and Pap [11, Theorem 4.1].

A.2 Theorem. Let us consider the two-factor affine diffusion model (1.1) with $a, b \in \mathbb{R}_{++}, \alpha, \beta \in \mathbb{R}$, $\gamma \in \mathbb{R}_{++}, \sigma_{1} \in \mathbb{R}_{++}, \sigma_{2}, \sigma_{3} \in \mathbb{R}_{+}$and $\varrho \in[-1,1]$ with a random initial value $\left(\eta_{0}, \zeta_{0}\right)$ independent of $\left(W_{t}, B_{t}, L_{t}\right)_{t \in \mathbb{R}_{+}}$satisfying $\mathbb{P}\left(\eta_{0} \in \mathbb{R}_{+}\right)=1$. Suppose that $\left(1-\varrho^{2}\right) \sigma_{2}^{2}+\sigma_{3}^{2}>0$. Then the process $\left(Y_{t}, X_{t}\right)_{t \in \mathbb{R}_{+}}$is exponentially ergodic, namely, there exist $\delta \in \mathbb{R}_{++}, B \in \mathbb{R}_{++}$and $\kappa \in \mathbb{R}_{++}$, such that

$$
\sup _{|g| \leqslant V+1}\left|\mathbb{E}\left(g\left(Y_{t}, X_{t}\right) \mid\left(Y_{0}, X_{0}\right)=\left(y_{0}, x_{0}\right)\right)-\mathbb{E}\left(g\left(Y_{\infty}, X_{\infty}\right)\right)\right| \leqslant B\left(V\left(y_{0}, x_{0}\right)+1\right) \mathrm{e}^{-\delta t}
$$


for all $t \in \mathbb{R}_{+}$and $\left(y_{0}, x_{0}\right) \in \mathbb{R}_{+} \times \mathbb{R}$, where the supremum is running for Borel measurable functions $g: \mathbb{R}_{+} \times \mathbb{R} \rightarrow \mathbb{R}$,

$$
V(y, x):=y^{2}+\kappa x^{2}, \quad(y, x) \in \mathbb{R}_{+} \times \mathbb{R},
$$

and the distribution of $\left(Y_{\infty}, X_{\infty}\right)$ is given by (A.1) and (A.2). Moreover, for all Borel measurable functions $f: \mathbb{R}^{2} \rightarrow \mathbb{R}$ with $\mathbb{E}\left(\left|f\left(Y_{\infty}, X_{\infty}\right)\right|\right)<\infty$, we have

$$
\mathbb{P}\left(\lim _{T \rightarrow \infty} \frac{1}{T} \int_{0}^{T} f\left(Y_{s}, X_{s}\right) \mathrm{d} s=\mathbb{E}\left(f\left(Y_{\infty}, X_{\infty}\right)\right)\right)=1 .
$$

\section{B Moments}

The next proposition gives a recursive formula for the moments of the process $\left(Y_{t}, X_{t}\right)_{t \in \mathbb{R}_{+}}$.

B.1 Proposition. Let us consider the two-factor affine diffusion model (1.1) with $a \in \mathbb{R}_{+}$, $b, \alpha, \beta, \gamma \in \mathbb{R}, \quad \sigma_{1}, \sigma_{2}, \sigma_{3} \in \mathbb{R}_{+}, \varrho \in[-1,1]$. Suppose that $\mathbb{E}\left(Y_{0}^{n}\left|X_{0}\right|^{p}\right)<\infty$ for some $n, p \in \mathbb{Z}_{+}$. Then for each $t \in \mathbb{R}_{+}$, we have $\mathbb{E}\left(Y_{t}^{k}\left|X_{t}\right|^{\ell}\right)<\infty$ for all $k \in\{0, \ldots, n\}$ and $\ell \in\{0, \ldots, p\}$, and the recursion

$$
\begin{aligned}
\mathbb{E}\left(Y_{t}^{k} X_{t}^{\ell}\right)= & \mathrm{e}^{-(k b+\ell \gamma) t} \mathbb{E}\left(Y_{0}^{k} X_{0}^{\ell}\right)+\left(k a+\frac{1}{2} k(k-1) \sigma_{1}^{2}\right) \int_{0}^{t} \mathrm{e}^{-(k b+\ell \gamma)(t-u)} \mathbb{E}\left(Y_{u}^{k-1} X_{u}^{\ell}\right) \mathrm{d} u \\
& +\left(\alpha+k \varrho \sigma_{1} \sigma_{2}\right) \int_{0}^{t} \mathrm{e}^{-(k b+\ell \gamma)(t-u)} \mathbb{E}\left(Y_{u}^{k} X_{u}^{\ell-1}\right) \mathrm{d} u-\ell \beta \int_{0}^{t} \mathrm{e}^{-(k b+\ell \gamma)(t-u)} \mathbb{E}\left(Y_{u}^{k+1} X_{u}^{\ell-1}\right) \mathrm{d} u \\
& +\frac{1}{2} \ell(\ell-1) \sigma_{2}^{2} \int_{0}^{t} \mathrm{e}^{-(k b+\ell \gamma)(t-u)} \mathbb{E}\left(Y_{u}^{k+1} X_{u}^{\ell-2}\right) \mathrm{d} u \\
& +\frac{1}{2} \ell(\ell-1) \sigma_{3}^{2} \int_{0}^{t} \mathrm{e}^{-(k b+\ell \gamma)(t-u)} \mathbb{E}\left(Y_{u}^{k} X_{u}^{\ell-2}\right) \mathrm{d} u
\end{aligned}
$$

for all $t \in \mathbb{R}_{+}$, where $\mathbb{E}\left(Y_{t}^{i} X_{t}^{j}\right):=0$ if $i, j \in \mathbb{Z}$ with $i<0$ or $j<0$. Especially,

$$
\begin{aligned}
\mathbb{E}\left(Y_{t}\right)= & \mathrm{e}^{-b t} \mathbb{E}\left(Y_{0}\right)+a \int_{0}^{t} \mathrm{e}^{-b(t-u)} \mathrm{d} u, \\
\mathbb{E}\left(X_{t}\right)= & \mathrm{e}^{-\gamma t} \mathbb{E}\left(X_{0}\right)+\alpha \int_{0}^{t} \mathrm{e}^{-\gamma(t-u)} \mathrm{d} u-\beta \int_{0}^{t} \mathrm{e}^{-\gamma(t-u)} \mathbb{E}\left(Y_{u}\right) \mathrm{d} u, \\
\mathbb{E}\left(Y_{t}^{2}\right)= & \mathrm{e}^{-2 b t} \mathbb{E}\left(Y_{0}^{2}\right)+\left(2 a+\sigma_{1}^{2}\right) \int_{0}^{t} \mathrm{e}^{-2 b(t-u)} \mathbb{E}\left(Y_{u}\right) \mathrm{d} u, \\
\mathbb{E}\left(Y_{t} X_{t}\right)= & \mathrm{e}^{-(b+\gamma) t} \mathbb{E}\left(Y_{0} X_{0}\right)+a \int_{0}^{t} \mathrm{e}^{-(b+\gamma)(t-u)} \mathbb{E}\left(X_{u}\right) \mathrm{d} u \\
& +\left(\alpha+\varrho \sigma_{1} \sigma_{2}\right) \int_{0}^{t} \mathrm{e}^{-(b+\gamma)(t-u)} \mathbb{E}\left(Y_{u}\right) \mathrm{d} u-\beta \int_{0}^{t} \mathrm{e}^{-(b+\gamma)(t-u)} \mathbb{E}\left(Y_{u}^{2}\right) \mathrm{d} u, \\
\mathbb{E}\left(X_{t}^{2}\right)= & \mathrm{e}^{-2 \gamma t} \mathbb{E}\left(X_{0}^{2}\right)+\alpha \int_{0}^{t} \mathrm{e}^{-2 \gamma(t-u)} \mathbb{E}\left(X_{u}\right) \mathrm{d} u-2 \beta \int_{0}^{t} \mathrm{e}^{-2 \gamma(t-u)} \mathbb{E}\left(Y_{u} X_{u}\right) \mathrm{d} u \\
& +\sigma_{2}^{2} \int_{0}^{t} \mathrm{e}^{-2 \gamma(t-u)} \mathbb{E}\left(Y_{u}\right) \mathrm{d} u+\sigma_{3}^{2} \int_{0}^{t} \mathrm{e}^{-2 \gamma(t-u)} \mathrm{d} u .
\end{aligned}
$$


If $\sigma_{1}>0$ and $Y_{0}=y_{0}$, then the Laplace transform of $Y_{t}, t \in \mathbb{R}_{++}$, takes the form

$$
\mathbb{E}\left(\mathrm{e}^{-\lambda Y_{t}}\right)=\left(1+\frac{\sigma_{1}^{2}}{2} \lambda \int_{0}^{t} \mathrm{e}^{-b u} \mathrm{~d} u\right)^{-\frac{2 a}{\sigma_{1}^{2}}} \exp \left\{-\frac{\lambda \mathrm{e}^{-b t} y_{0}}{1+\frac{\sigma_{1}^{2}}{2} \lambda \int_{0}^{t} \mathrm{e}^{-b u} \mathrm{~d} u}\right\}, \quad \lambda \in \mathbb{R}_{+},
$$

i.e., $Y_{t}$ has a non-centered chi-square distribution up to a multiplicative constant $\frac{\sigma_{1}^{2}}{4} \int_{0}^{t} \mathrm{e}^{-b u} \mathrm{~d} u$, with degrees of freedom $\frac{4 a}{\sigma_{1}^{2}}$ and with non-centrality parameter $\frac{4 \mathrm{e}^{-b t} y_{0}}{\sigma_{1}^{2} \int_{0}^{t} \mathrm{e}^{-b u} \mathrm{~d} u}$.

If $\sigma_{1}>0$ and $\left(1-\varrho^{2}\right) \sigma_{2}^{2}+\sigma_{3}^{2}>0$, then for each $t \in \mathbb{R}_{++}$, the distribution of $\left(Y_{t}, X_{t}\right)$ is absolutely continuous.

Proof. It is sufficient to prove the recursion in the case when $\left(Y_{0}, X_{0}\right)=\left(y_{0}, x_{0}\right)$ with an arbitrary $\left(y_{0}, x_{0}\right) \in \mathbb{R}_{++} \times \mathbb{R}$, since then, for arbitrary initial values with $\mathbb{E}\left(Y_{0}^{n}\left|X_{0}\right|^{p}\right)<\infty$, the recursion follows by the law of total expectation. One can show that

$$
\int_{0}^{t} \mathbb{E}\left(Y_{u}^{k} X_{u}^{2 \ell}\right) \mathrm{d} u<\infty \quad \text { for all } t \in \mathbb{R}_{+} \text {and } k, \ell \in \mathbb{Z}_{+},
$$

see Bolyog and Pap [11, proof of Theorem 5.1]. For all $k, \ell \in \mathbb{Z}_{+}$, using the independence of $W, B$ and $L$, by Itô's formula, we have

$$
\begin{aligned}
\mathrm{d}\left(\mathrm{e}^{(k b+\ell \gamma) t} Y_{t}^{k} X_{t}^{\ell}\right) \\
=(k b+\ell \gamma) \mathrm{e}^{(k b+\ell \gamma) t} Y_{t}^{k} X_{t}^{\ell} \mathrm{d} t+k \mathrm{e}^{(k b+\ell \gamma) t} Y_{t}^{k-1} X_{t}^{\ell}\left[\left(a-b Y_{t}\right) \mathrm{d} t+\sigma_{1} \sqrt{Y_{t}} \mathrm{~d} W_{t}\right] \\
\quad+\ell \mathrm{e}^{(k b+\ell \gamma) t} Y_{t}^{k} X_{t}^{\ell-1}\left[\left(\alpha-\beta Y_{t}-\gamma X_{t}\right) \mathrm{d} t+\sigma_{2} \sqrt{Y_{t}}\left(\varrho \mathrm{d} W_{t}+\sqrt{1-\varrho^{2}} \mathrm{~d} B_{t}\right)+\sigma_{3} \mathrm{~d} L_{t}\right] \\
\quad+\frac{1}{2} k(k-1) \mathrm{e}^{(k b+\ell \gamma) t} Y_{t}^{k-2} X_{t}^{\ell} \sigma_{1}^{2} Y_{t} \mathrm{~d} t+k \ell \mathrm{e}^{-(k b+\ell \gamma) t} Y_{t}^{k-1} X_{t}^{\ell-1} \varrho \sigma_{1} \sigma_{2} Y_{t} \mathrm{~d} t \\
+\frac{1}{2} \ell(\ell-1) \mathrm{e}^{(k b+\ell \gamma) t} Y_{t}^{k} X_{t}^{\ell-2}\left(\sigma_{2}^{2} Y_{t}+\sigma_{3}^{2}\right) \mathrm{d} t \\
=k \mathrm{e}^{(k b+\ell \gamma) t} Y_{t}^{k-1} X_{t}^{\ell}\left(a \mathrm{~d} t+\sigma_{1} \sqrt{Y_{t}} \mathrm{~d} W_{t}\right) \\
+\ell \mathrm{e}^{(k b+\ell \gamma) t} Y_{t}^{k} X_{t}^{\ell-1}\left[\left(\alpha-\beta Y_{t}\right) \mathrm{d} t+\sigma_{2} \sqrt{Y_{t}}\left(\varrho \mathrm{d} W_{t}+\sqrt{1-\varrho^{2}} \mathrm{~d} B_{t}\right)+\sigma_{3} \mathrm{~d} L_{t}\right] \\
+\frac{1}{2} k(k-1) \sigma_{1}^{2} \mathrm{e}^{(k b+\ell \gamma) t} Y_{t}^{k-1} X_{t}^{\ell} \mathrm{d} t+k \ell \varrho \sigma_{1} \sigma_{2} \mathrm{e}^{(k b+\ell \gamma) t} Y_{t}^{k} X_{t}^{\ell-1} \mathrm{~d} t \\
+\frac{1}{2} \ell(\ell-1) \sigma_{2}^{2} \mathrm{e}^{(k b+\ell \gamma) t} Y_{t}^{k+1} X_{t}^{\ell-2} \mathrm{~d} t+\frac{1}{2} \ell(\ell-1) \sigma_{3}^{2} \mathrm{e}^{(k b+\ell \gamma) t} Y_{t}^{k} X_{t}^{\ell-2} \mathrm{~d} t .
\end{aligned}
$$

Writing this in an integrated form and taking the expectation of both sides, we obtain the recursive formulas for $\mathbb{E}\left(Y_{t}^{k}\left|X_{t}\right|^{\ell}\right)<\infty$ for all $k \in\{0, \ldots, n\}$ and $\ell \in\{0, \ldots, p\}$.

Formula (B.1) can be found in Ikeda and Watanabe [22, Example 8.2].

If $\sigma_{1}>0$ and $\left(1-\varrho^{2}\right) \sigma_{2}^{2}+\sigma_{3}^{2}>0$, then for each $t \in \mathbb{R}_{++}$, the conditional distribution of $\left(Y_{t}, X_{t}\right)$ given $\left(Y_{0}, X_{0}\right)$ is absolutely continuous, see the proof of part (b) in the proof of Theorem 3.1 in Bolyog and Pap [1]. This clearly implies that the (unconditional) distribution of $\left(Y_{t}, X_{t}\right)$ is absolutely continuous.

The next theorem gives a recursive formula for the moments of the stationary distribution of the process $\left(Y_{t}, X_{t}\right)_{t \in \mathbb{R}_{+}}$in the subcritical case, see Bolyog and Pap [11, Theorem 5.1]. 
B.2 Theorem. Let us consider the two-factor affine diffusion model (1.1) with $a \in \mathbb{R}_{+}, \quad b \in \mathbb{R}_{++}$, $\alpha, \beta \in \mathbb{R}, \quad \gamma \in \mathbb{R}_{++}, \quad \sigma_{1}, \sigma_{2}, \sigma_{3} \in \mathbb{R}_{+}, \varrho \in[-1,1]$, and the random vector $\left(Y_{\infty}, X_{\infty}\right)$ given by Theorem A.1. Then all the (mixed) moments of $\left(Y_{\infty}, X_{\infty}\right)$ of any order are finite, i.e., we have $\mathbb{E}\left(Y_{\infty}^{n}\left|X_{\infty}\right|^{p}\right)<\infty$ for all $n, p \in \mathbb{Z}_{+}$, and the recursion

$$
\begin{aligned}
\mathbb{E}\left(Y_{\infty}^{n} X_{\infty}^{p}\right)=\frac{1}{n b+p \gamma}[ & \left(n a+\frac{1}{2} n(n-1) \sigma_{1}^{2}\right) \mathbb{E}\left(Y_{\infty}^{n-1} X_{\infty}^{p}\right)-p \beta \mathbb{E}\left(Y_{\infty}^{n+1} X_{\infty}^{p-1}\right) \\
& +p\left(\alpha+n \varrho \sigma_{1} \sigma_{2}\right) \mathbb{E}\left(Y_{\infty}^{n} X_{\infty}^{p-1}\right)+\frac{1}{2} p(p-1) \sigma_{2}^{2} \mathbb{E}\left(Y_{\infty}^{n+1} X_{\infty}^{p-2}\right) \\
& \left.+\frac{1}{2} p(p-1) \sigma_{3}^{2} \mathbb{E}\left(Y_{\infty}^{n} X_{\infty}^{p-2}\right)\right],
\end{aligned}
$$

holds for all $n, p \in \mathbb{Z}_{+}$with $n+p \geqslant 1$, where $\mathbb{E}\left(Y_{\infty}^{k} X_{\infty}^{\ell}\right):=0$ for $k, \ell \in \mathbb{Z}$ with $k<0$ or $\ell<0$. Especially,

$$
\begin{gathered}
\mathbb{E}\left(Y_{\infty}\right)=\frac{a}{b}, \quad \mathbb{E}\left(Y_{\infty}^{2}\right)=\frac{a\left(2 a+\sigma_{1}^{2}\right)}{2 b^{2}}, \quad \mathbb{E}\left(Y_{\infty}^{3}\right)=\frac{a\left(a+\sigma_{1}^{2}\right)\left(2 a+\sigma_{1}^{2}\right)}{2 b^{3}}, \\
\mathbb{E}\left(X_{\infty}\right)=\frac{b \alpha-a \beta}{b \gamma}, \quad \mathbb{E}\left(Y_{\infty} X_{\infty}\right)=\frac{a \mathbb{E}\left(X_{\infty}\right)-\beta \mathbb{E}\left(Y_{\infty}^{2}\right)+\left(\alpha+\varrho \sigma_{1} \sigma_{2}\right) \mathbb{E}\left(Y_{\infty}\right)}{b+\gamma}, \\
\mathbb{E}\left(X_{\infty}^{2}\right)=\frac{-2 \beta \mathbb{E}\left(Y_{\infty} X_{\infty}\right)+2 \alpha \mathbb{E}\left(X_{\infty}\right)+\sigma_{2}^{2} \mathbb{E}\left(Y_{\infty}\right)+\sigma_{3}^{2}}{2 \gamma}, \\
\mathbb{E}\left(Y_{\infty}^{2} X_{\infty}\right)=\frac{\left(2 a+\sigma_{1}^{2}\right) \mathbb{E}\left(Y_{\infty} X_{\infty}\right)-\beta \mathbb{E}\left(Y_{\infty}^{3}\right)+\left(\alpha+2 \varrho \sigma_{1} \sigma_{2}\right) \mathbb{E}\left(Y_{\infty}^{2}\right)}{2 b+\gamma}, \\
\mathbb{E}\left(Y_{\infty} X_{\infty}^{2}\right)=\frac{a \mathbb{E}\left(X_{\infty}^{2}\right)-2 \beta \mathbb{E}\left(Y_{\infty}^{2} X_{\infty}\right)+2\left(\alpha+\varrho \sigma_{1} \sigma_{2}\right) \mathbb{E}\left(Y_{\infty} X_{\infty}\right)+\sigma_{2}^{2} \mathbb{E}\left(Y_{\infty}^{2}\right)+\sigma_{3}^{2} \mathbb{E}\left(Y_{\infty}\right)}{b+2 \gamma} .
\end{gathered}
$$

If $\sigma_{1}>0$, then the Laplace transform of $Y_{\infty}$ takes the form

$$
\mathbb{E}\left(\mathrm{e}^{-\lambda Y_{\infty}}\right)=\left(1+\frac{\sigma_{1}^{2}}{2 b} \lambda\right)^{-2 a / \sigma_{1}^{2}}, \quad \lambda \in \mathbb{R}_{+},
$$

i.e., $Y_{\infty}$ has gamma distribution with parameters $2 a / \sigma_{1}^{2}$ and $2 b / \sigma_{1}^{2}$, hence

$$
\mathbb{E}\left(Y_{\infty}^{\kappa}\right)=\frac{\Gamma\left(\frac{2 a}{\sigma_{1}^{2}}+\kappa\right)}{\left(\frac{2 b}{\sigma_{1}^{2}}\right)^{\kappa} \Gamma\left(\frac{2 a}{\sigma_{1}^{2}}\right)}, \quad \kappa \in\left(-\frac{2 a}{\sigma_{1}^{2}}, \infty\right) .
$$

If $\sigma_{1}>0$ and $\left(1-\varrho^{2}\right) \sigma_{2}^{2}+\sigma_{3}^{2}>0$, then the distribution of $\left(Y_{\infty}, X_{\infty}\right)$ is absolutely continuous.

\section{Statistics for diffusion coefficients}

Next, for any $T>0$, we give a statistic for $\sigma_{1}^{2}, \sigma_{2}^{2}, \sigma_{3}^{2}$ and $\varrho$ using continuous time observations $\left(Y_{t}, X_{t}\right)_{t \in[0, T]}$. Due to this result, we do not consider the estimation of the parameters $\sigma_{1}, \sigma_{2}, \sigma_{3}$ and $\varrho$, they are supposed to be known. 
Let us consider the two-factor affine diffusion model (1.1) with $a \in \mathbb{R}_{+}, b, \alpha, \beta, \gamma \in \mathbb{R}, \sigma_{1} \in \mathbb{R}_{++}$, $\sigma_{2}, \sigma_{3} \in \mathbb{R}_{+}, \varrho \in[-1,1]$. Suppose that we have $\mathbb{P}\left(Y_{0} \in \mathbb{R}_{++}\right)$or $a \in \mathbb{R}_{++}$. Then for all $T \in \mathbb{R}_{++}$, we have

$$
\mathbb{P}\left(\int_{0}^{T} Y_{u} \mathrm{~d} u \in \mathbb{R}_{++}\right)=1 .
$$

Indeed, if $\omega \in \Omega$ is such that $[0, t] \ni u \mapsto Y_{u}(\omega)$ is continuous and $Y_{v}(\omega) \in \mathbb{R}_{+}$for all $v \in \mathbb{R}_{+}$, then we have $\int_{0}^{t} Y_{s}(\omega) \mathrm{d} s=0$ if and only if $Y_{s}(\omega)=0$ for all $s \in[0, t]$. Using the method of the proof of Theorem 3.1 in Barczy et. al [5], we get (C.1). The (predictable) quadratic variation process of $Y, X$, and the (predictable) quadratic covariation process of $Y$ and $X$ are

$$
\langle Y\rangle_{t}=\sigma_{1}^{2} \int_{0}^{t} Y_{u} \mathrm{~d} u, \quad\langle X\rangle_{t}=\sigma_{2}^{2} \int_{0}^{t} Y_{u} \mathrm{~d} u+\sigma_{3}^{2} t, \quad\langle Y, X\rangle_{t}=\varrho \sigma_{1} \sigma_{2} \int_{0}^{t} Y_{u} \mathrm{~d} u, \quad t \in \mathbb{R}_{+} .
$$

If, in addition, $a \in\left(\sigma_{1}^{2}, \infty\right)$, then for each $T \in \mathbb{R}_{++}$, we have

$$
\begin{gathered}
{\left[\begin{array}{l}
\sigma_{2}^{2} \\
\sigma_{3}^{2}
\end{array}\right]=\left[\begin{array}{cc}
\int_{0}^{T} Y_{u} \mathrm{~d} u & T \\
\int_{0}^{T / 2} Y_{u} \mathrm{~d} u & T / 2
\end{array}\right]^{-1}\left[\begin{array}{c}
\langle X\rangle_{T} \\
\langle X\rangle_{T / 2}
\end{array}\right]=:\left[\begin{array}{l}
\widehat{\sigma}_{2}^{2}(T) \\
\widehat{\sigma}_{3}^{2}(T)
\end{array}\right],} \\
\sigma_{1}^{2}=\frac{\langle Y\rangle_{T}}{\int_{0}^{T} Y_{u} \mathrm{~d} u}=: \widehat{\sigma}_{1}^{2}(T), \quad \varrho=\frac{\langle Y, X\rangle_{T}}{\sigma_{1} \sigma_{2} \int_{0}^{T} Y_{u} \mathrm{~d} u}=\frac{\langle Y, X\rangle_{T}}{\left(\widehat{\sigma}_{1}^{2}(T) \widehat{\sigma}_{2}^{2}(T)\right)^{\frac{1}{2}} \int_{0}^{T} Y_{u} \mathrm{~d} u}=: \widehat{\varrho}(T),
\end{gathered}
$$

since the matrix

$$
\left[\begin{array}{cc}
\int_{0}^{T} Y_{u} \mathrm{~d} u & T \\
\int_{0}^{T / 2} Y_{u} \mathrm{~d} u & T / 2
\end{array}\right]
$$

is invertible almost surely. Indeed,

$$
\begin{aligned}
& \mathbb{P}\left(\frac{T}{2} \int_{0}^{T} Y_{u} \mathrm{~d} u-T \int_{0}^{T / 2} Y_{u} \mathrm{~d} u=0\right)=\mathbb{P}\left(\int_{T / 2}^{T} Y_{u} \mathrm{~d} u=\int_{0}^{T / 2} Y_{u} \mathrm{~d} u\right) \\
& =\mathbb{E}\left(\mathbb{P}\left(\int_{T / 2}^{T} Y_{u} \mathrm{~d} u=\int_{0}^{T / 2} Y_{u} \mathrm{~d} u \mid\left(Y_{u}\right)_{u \in[0, T / 2]}\right)\right) \\
& =\mathbb{E}\left(\left.\mathbb{P}\left(\int_{T / 2}^{T} Y_{u} \mathrm{~d} u=I \mid\left(Y_{u}\right)_{u \in[0, T / 2]}\right)\right|_{I=\int_{0}^{T / 2} Y_{u} \mathrm{~d} u}\right) \\
& =\mathbb{E}\left(\left.\mathbb{P}\left(\int_{T / 2}^{T} Y_{u} \mathrm{~d} u=I \mid Y_{T / 2}=y\right)\right|_{I=\int_{0}^{T / 2} Y_{u} \mathrm{~d} u, y=Y_{T / 2}}\right) \\
& =\mathbb{E}\left(\left.\mathbb{P}\left(\int_{0}^{T / 2} Y_{u} \mathrm{~d} u=I \mid Y_{0}=y\right)\right|_{I=\int_{0}^{T / 2} Y_{u} \mathrm{~d} u, y=Y_{T / 2}}\right)=0,
\end{aligned}
$$

since

$$
\mathbb{P}\left(\int_{0}^{T / 2} Y_{u} \mathrm{~d} u=I \mid Y_{0}=y\right)=0
$$

for each $I \in \mathbb{R}_{+}$and $y \in \mathbb{R}_{+}$, because the additional condition $a \in\left(\sigma_{1}^{2}, \infty\right)$ yields that the distribution of $\int_{0}^{T / 2} Y_{u} \mathrm{~d} u$ is absolutely continuous, see Filipović et al. [18, Theorem 4.3]. (The absolute continuity of $\int_{0}^{T / 2} Y_{u} \mathrm{~d} u$ might hold without the additional condition $a \in\left(\sigma_{1}^{2}, \infty\right)$.) 
We note that $\left(\widehat{\sigma}_{1}^{2}(T), \widehat{\sigma}_{2}^{2}(T), \widehat{\sigma}_{3}^{2}(T), \widehat{\varrho}(T)\right)$ is a statistic, i.e., there exists a measurable function $\Xi: D([0, T], \mathbb{R}) \rightarrow \mathbb{R}^{4}$ such that $\left(\widehat{\sigma}_{1}^{2}(T), \widehat{\sigma}_{2}^{2}(T), \widehat{\sigma}_{3}^{2}(T), \widehat{\varrho}(T)\right)=\Xi\left(\left(Y_{u}\right)_{u \in[0, T]}\right)$, where $D([0, T], \mathbb{R})$ denotes the space of real-valued càdlàg functions defined on $[0, T]$, since

$$
\begin{gathered}
{\left[\begin{array}{cc}
\frac{1}{n} \sum_{i=1}^{\lfloor n T\rfloor} Y_{\frac{i-1}{n}} & T \\
\frac{1}{n} \sum_{i=1}^{\lfloor n T\rfloor / 2} Y_{\frac{i-1}{n}} & T / 2
\end{array}\right]^{-1}\left[\begin{array}{c}
\sum_{i=1}^{\lfloor n T\rfloor}\left(X_{\frac{i}{n}}-X_{\frac{i-1}{n}}\right)^{2} \\
\sum_{i=1}^{\lfloor n T\rfloor / 2}\left(X_{\frac{i}{n}}-X_{\frac{i-1}{n}}\right)^{2}
\end{array}\right] \stackrel{\mathbb{P}}{\longrightarrow}\left[\begin{array}{c}
\widehat{\sigma}_{2}^{2}(T) \\
\widehat{\sigma}_{3}^{2}(T)
\end{array}\right],} \\
\frac{\sum_{i=1}^{\lfloor n T\rfloor}\left(Y_{\frac{i}{n}}-Y_{\frac{i-1}{n}}\right)^{2}}{\frac{1}{n} \sum_{i=1}^{\lfloor n T\rfloor} Y_{\frac{i-1}{n}}} \stackrel{\mathbb{P}}{\longrightarrow} \widehat{\sigma}_{1}^{2}(T), \quad \frac{\sum_{i=1}^{\lfloor n T\rfloor}\left(Y_{\frac{i}{n}}-Y_{\frac{i-1}{n}}\right)\left(X_{\frac{i}{n}}-X_{\frac{i-1}{n}}\right)}{\frac{1}{n} \sum_{i=1}^{\lfloor n T\rfloor} Y_{\frac{i-1}{n}}} \stackrel{\mathbb{P}}{\longrightarrow} \widehat{\varrho}(T)
\end{gathered}
$$

as $n \rightarrow \infty$, where the convergences in (C.2) and (C.3) hold almost surely along a suitable subsequence, the members of the sequences in (C.2) and (C.3) are measurable functions of $\left(Y_{u}, X_{u}\right)_{u \in[0, T]}$, and one can use Theorems 4.2.2 and 4.2.8 in Dudley [15]. Next we prove (C.2) and (C.3). By Theorems I.4.47 a) and I.4.52 in Jacod and Shiryaev [23],

$$
\begin{gathered}
\sum_{i=1}^{\lfloor n T\rfloor}\left(X_{\frac{i}{n}}-X_{\frac{i-1}{n}}\right)^{2} \stackrel{\mathbb{P}}{\longrightarrow}[X]_{T}=\langle X\rangle_{T}, \quad \sum_{i=1}^{\lfloor n T\rfloor / 2}\left(X_{\frac{i}{n}}-X_{\frac{i-1}{n}}\right)^{2} \stackrel{\mathbb{P}}{\longrightarrow}[X]_{T / 2}=\langle X\rangle_{T / 2}, \\
\sum_{i=1}^{\lfloor n T\rfloor}\left(Y_{\frac{i}{n}}-Y_{\frac{i-1}{n}}\right)^{2} \stackrel{\mathbb{P}}{\longrightarrow}[Y]_{T}=\langle Y\rangle_{T}, \quad \sum_{i=1}^{\lfloor n T\rfloor}\left(Y_{\frac{i}{n}}-Y_{\frac{i-1}{n}}\right)\left(X_{\frac{i}{n}}-X_{\frac{i-1}{n}}\right) \stackrel{\mathbb{P}}{\longrightarrow}[Y, X]_{T}=\langle Y, X\rangle_{T}
\end{gathered}
$$

as $n \rightarrow \infty$. Moreover, for all $T \in \mathbb{R}_{+}$, we have

$$
\frac{1}{n} \sum_{i=1}^{\lfloor n T\rfloor} Y_{\frac{i-1}{n}} \stackrel{\mathbb{P}}{\longrightarrow} \int_{0}^{T} Y_{u} \mathrm{~d} u, \quad \frac{1}{n} \sum_{i=1}^{\lfloor n T\rfloor / 2} Y_{\frac{i-1}{n}} \stackrel{\mathbb{P}}{\longrightarrow} \int_{0}^{T / 2} Y_{u} \mathrm{~d} u
$$

as $n \rightarrow \infty$, see Proposition I.4.44 in Jacod and Shiryaev [23]. Hence (C.2) and (C.3) follow by Slutsky's lemma.

\section{Limit theorems for continuous local martingales}

In what follows we recall some limit theorems for continuous local martingales. We use these limit theorems for studying the asymptotic behaviour of the MLE of $\boldsymbol{\theta}=(a, b, \alpha, \beta, \gamma)^{\top}$. First we recall a strong law of large numbers for continuous local martingales.

D.1 Theorem. (Liptser and Shiryaev [26, Lemma 17.4]) Let $\left(\Omega, \mathcal{F},\left(\mathcal{F}_{t}\right)_{t \in \mathbb{R}_{+}}, \mathbb{P}\right)$ be a filtered probability space satisfying the usual conditions. Let $\left(M_{t}\right)_{t \in \mathbb{R}_{+}}$be a square-integrable continuous local martingale with respect to the filtration $\left(\mathcal{F}_{t}\right)_{t \in \mathbb{R}_{+}}$such that $\mathbb{P}\left(M_{0}=0\right)=1$. Let $\left(\xi_{t}\right)_{t \in \mathbb{R}_{+}}$be a progressively measurable process such that

$$
\mathbb{P}\left(\int_{0}^{t} \xi_{u}^{2} \mathrm{~d}\langle M\rangle_{u}<\infty\right)=1, \quad t \in \mathbb{R}_{+},
$$

and

$$
\int_{0}^{t} \xi_{u}^{2} \mathrm{~d}\langle M\rangle_{u} \stackrel{\text { a.s. }}{\longrightarrow} \infty \quad \text { as } t \rightarrow \infty
$$


where $\left(\langle M\rangle_{t}\right)_{t \in \mathbb{R}_{+}}$denotes the quadratic variation process of $M$. Then

$$
\frac{\int_{0}^{t} \xi_{u} \mathrm{~d} M_{u}}{\int_{0}^{t} \xi_{u}^{2} \mathrm{~d}\langle M\rangle_{u}} \stackrel{\text { a.s. }}{\longrightarrow} 0 \quad \text { as } t \rightarrow \infty \text {. }
$$

If $\left(M_{t}\right)_{t \in \mathbb{R}_{+}}$is a standard Wiener process, the progressive measurability of $\left(\xi_{t}\right)_{t \in \mathbb{R}_{+}}$can be relaxed to measurability and adaptedness to the filtration $\left(\mathcal{F}_{t}\right)_{t \in \mathbb{R}_{+}}$.

The next theorem is about the asymptotic behaviour of continuous multivariate local martingales, see van Zanten [29, Theorem 4.1].

D.2 Theorem. (van Zanten [29, Theorem 4.1]) Let $\left(\Omega, \mathcal{F},\left(\mathcal{F}_{t}\right)_{t \in \mathbb{R}_{+}}, \mathbb{P}\right)$ be a filtered probability space satisfying the usual conditions. Let $\left(\boldsymbol{M}_{t}\right)_{t \in \mathbb{R}_{+}}$be a d-dimensional square-integrable continuous local martingale with respect to the filtration $\left(\mathcal{F}_{t}\right)_{t \in \mathbb{R}_{+}}$such that $\mathbb{P}\left(\boldsymbol{M}_{0}=\mathbf{0}\right)=1$. Suppose that there exists a function $\boldsymbol{Q}:\left[t_{0}, \infty\right) \rightarrow \mathbb{R}^{d \times d}$ with some $t_{0} \in \mathbb{R}_{+}$such that $\boldsymbol{Q}(t)$ is an invertible (non-random) matrix for all $t \in \mathbb{R}_{+}, \lim _{t \rightarrow \infty}\|\boldsymbol{Q}(t)\|=0$ and

$$
\boldsymbol{Q}(t)\langle\boldsymbol{M}\rangle_{t} \boldsymbol{Q}(t)^{\top} \stackrel{\mathbb{P}}{\longrightarrow} \boldsymbol{\eta} \boldsymbol{\eta}^{\top} \quad \text { as } t \rightarrow \infty,
$$

where $\boldsymbol{\eta}$ is a $d \times d$ random matrix. Then, for each $\mathbb{R}^{k \times \ell}$-valued random matrix $\boldsymbol{A}$ defined on $(\Omega, \mathcal{F}, \mathbb{P})$, we have

$$
\left(\boldsymbol{Q}(t) \boldsymbol{M}_{t}, \boldsymbol{A}\right) \stackrel{\mathcal{D}}{\longrightarrow}(\boldsymbol{\eta} \boldsymbol{Z}, \boldsymbol{A}) \quad \text { as } t \rightarrow \infty,
$$

where $\boldsymbol{Z}$ is a d-dimensional standard normally distributed random vector independent of $(\boldsymbol{\eta}, \boldsymbol{A})$.

\section{References}

[1] Alfonsi, A. (2015). Affine Diffusions and Related Processes: Simulation, Theory and Applications. Springer, Cham, Bocconi University Press, Milan.

[2] Baldeaux, J. and Platen, E. (2013). Functionals of Multidimensional Diffusions with Applications to Finance. Springer, Cham; Bocconi University Press, Milan.

[3] Barczy, M., Ben Alaya, M., Kebaier, A. and Pap, G. (2016). Asymptotic behavior of maximum likelihood estimators for a jump-type Heston model. Available on ArXiv: http://arxiv.org/abs/1509.08869

[4] Barczy, M., Ben Alaya, M., Kebaier, A. and Pap, G. (2017+). Asymptotic properties of maximum likelihood estimator for the growth rate for a jump-type CIR process based on continuous time observations. To appear in Stochastic Processes and their Applications. Available on ArXiv: http://arxiv.org/abs/1609.05865

[5] Barczy, M., Döring, L., Li, Z. and PAP, G. (2013). On parameter estimation for critical affine processes. Electronic Journal of Statistics 7 647-696.

[6] Barczy, M., Döring, L., Li, Z. and Pap, G. (2014). Parameter estimation for a subcritical affine two factor model. Journal of Statistical Planning and Inference 151-152 37-59. 
[7] Barczy, M., Nyul, B. and PAP, G. (2016). Least squares estimation for the subcritical Heston model based on continuous time observations. Available at arXiv http://arxiv.org/abs/1511.05948

[8] Barczy, M. and PAP, G. (2016). Asymptotic properties of maximum-likelihood estimators for Heston models based on continuous time observations. Statistics 50(2) 389-417.

[9] Ben Alaya, M. and Kebaier, A. (2012). Parameter estimation for the square root diffusions: ergodic and nonergodic cases. Stochastic Models 28(4) 609-634.

[10] Ben Alaya, M. and Kebaier, A. (2013). Asymptotic behavior of the maximum likelihood estimator for ergodic and nonergodic square-root diffusions. Stochastic Analysis and Applications 31(4) $552-573$.

[11] Bolyog, B. and PAP, G. (2016). Conditions for stationarity and ergodicity of two-factor affine diffusions. Communications on Stochastic Analysis 10(4) 587-610.

[12] Cox, J. C., Ingersoll, J. E. and Ross, S. A. (1985). A theory of the term structure of interest rates. Econometrica 53(2) 385-407.

[13] Dawson, D. A. and Li, Z.: Skew convolution semigroups and affine Markov processes, Ann. Probab. 34(3) (2006) 1103-1142.

[14] Dietz, H. M. and Kutoyants, Yu. A. (1997). A class of minimum-distance estimators for diffusion processes with ergodic properties. Statistics and Decisions 15 211-227.

[15] Dudley, R. M. (1989). Real Analysis and Probability. Wadsworth \& Brooks/Cole Advanced Books \& Software, Pacific Grove, California.

[16] Duffie, D., Filipović, D. and Schachermayer, W. (2003). Affine processes and applications in finance. Annals of Applied Probability 13 984-1053.

[17] Filipović, D. (2009). Term-Structure Models. Springer-Verlag, Berlin.

[18] Filipović, D., Mayerhofer, E. and Schneider, P. (2013). Density approximations for multivariate affine jump-diffusion processes. Journal of Econometrics 176 93-111.

[19] Hu, Y. and Long, H. (2007). Parameter estimation for Ornstein-Uhlenbeck processes driven by $\alpha$-stable Lévy motions. Communications on Stochastic Analysis 1(2) 175-192.

[20] Hu, Y. and Long, H. (2009). Least squares estimator for Ornstein-Uhlenbeck processes driven by $\alpha$-stable motions. Stochastic Processes and their Applications 119(8) 2465-2480.

[21] Hu, Y. and Long, H. (2009). On the singularity of least squares estimator for mean-reverting $\alpha$-stable motions. Acta Mathematica Scientia 29B(3) 599-608.

[22] Ikeda, N. and Watanabe, S. (1981). Stochastic Differential Equations and Diffusion Processes. North-Holland Publishing Company.

[23] Jacod, J. and Shiryaev, A. N. (2003). Limit Theorems for Stochastic Processes, 2nd ed. Springer-Verlag, Berlin. 
[24] Karatzas, I. and Shreve, S. E. (1991). Brownian Motion and Stochastic Calculus, 2nd ed. Springer-Verlag, New York.

[25] KüChler, U. and Sørensen, M. (1997). Exponential families of stochastic processes, SpringerVerlag, New York.

[26] Liptser, R. S. and Shiryaev, A. N. (2001). Statistics of Random Processes II. Applications, 2nd edition. Springer-Verlag, Berlin, Heidelberg.

[27] Overbeck, L. and RydÉn, T. (1997). Estimation in the Cox-Ingersoll-Ross model. Econometric Theory 13(3) 430-461.

[28] van der VaArt, A. W. (1998). Asymptotic Statistics, Cambridge University Press.

[29] VAn Zanten, H. (2000). A multivariate central limit theorem for continuous local martingales. Statistics 83 Probability Letters 50(3) 229-235. 